\title{
LA TÉRATOlOGIE DES TIQUES (fin)
}

\author{
Par Yyonne CAMPANA-ROUGET
}

\section{A nom alies locales}

\section{Anomalies du corps}

\section{Anomalies du scutum}

Hyalomma anatolicum. Pervomaisky 1954.

Deux femelles présentent des déformations importantes du scutum : l'une montre une échancrure profonde dans la région postérieure médiane du scutum ; chez l'autre, la partie droite du scutum manque et les yeux sont asymétriques.

\section{Asymétrie des stigmates}

Amblyomma hebraeum Koch. Mozambique. T. Santos Dias 1947.

Trois exemplaires. Les stigmates sont asymétriques et il manque des festons (fig. 61).
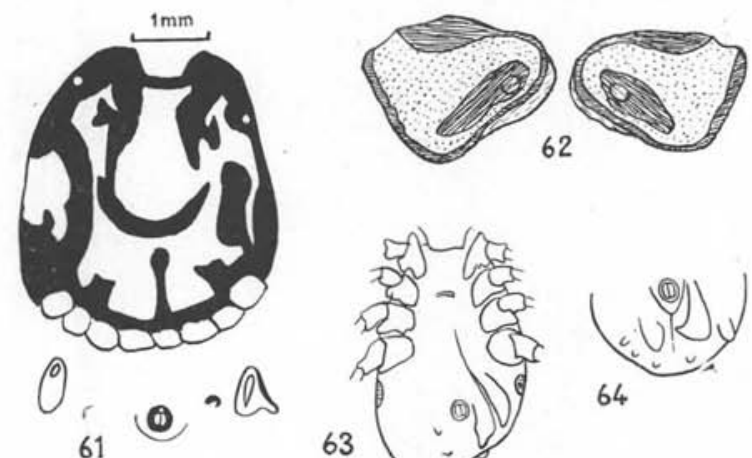

63
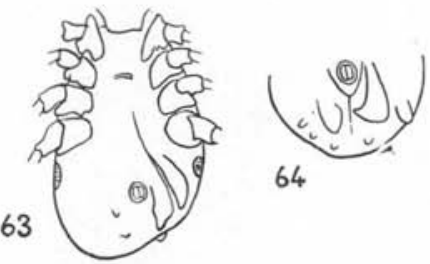

FIG. 61. - Amblyomma hebraeum. Asymétrie des stigmates. (D'après Santos Dias); Fig 62. - Amblyomma longirostrum. Asymétrie des stigmates. (D'après Cooper et Robinson) ; FIG. 63. - Boophilus australis. Asymétrie des écussons. (D'après Warburton et Nutta!I); Fig. 64. - Hyalomma sp. Asymétrie des écussons. (D'après Warburton et Nuttall), 
Amblyomma longirostrum Koch. Cooper et Robinson 1908 (fig. 62). Hyalomma dromedarii Koch. Brésil. Silva Leitao 1943.

Deux exemplaires.

\section{Anomalies des écussons}

\section{Asymétries :}

Boophilus australis (Fuller 1897). Indes. Warburton et Nuttall 1909 (fig. 63).

Hyalomma sp. Afrique du Sud. Warburton et Nuttall 1909 (fig. 64).

Hyalomma sp. Transvaal. Robinson 1920.

Hyalomma sp. Indes. Sharif 1930 (fig. 65).

Hyalomma sp. Russie. Pavlovsky 1940.

Hyalomma detritum Schulze. Russie. Pavlovsky 1940.

Hyalomma excavatum. Italie. Starkoff 1956 (fig. 66).

Hyalomma hussaini Sharif. Indes. Sharif 1930 (fig. 67).

Rhipicephalus ayrei Lewis 1933. Mozambique. T. Santos Dias 1948.

Rhipicephalus longiceps Warburton 1912. Angola. Nuttall 1915.

Rhipicephalus sanguineus. Indes. Nuttall 1915 (fig. 68).
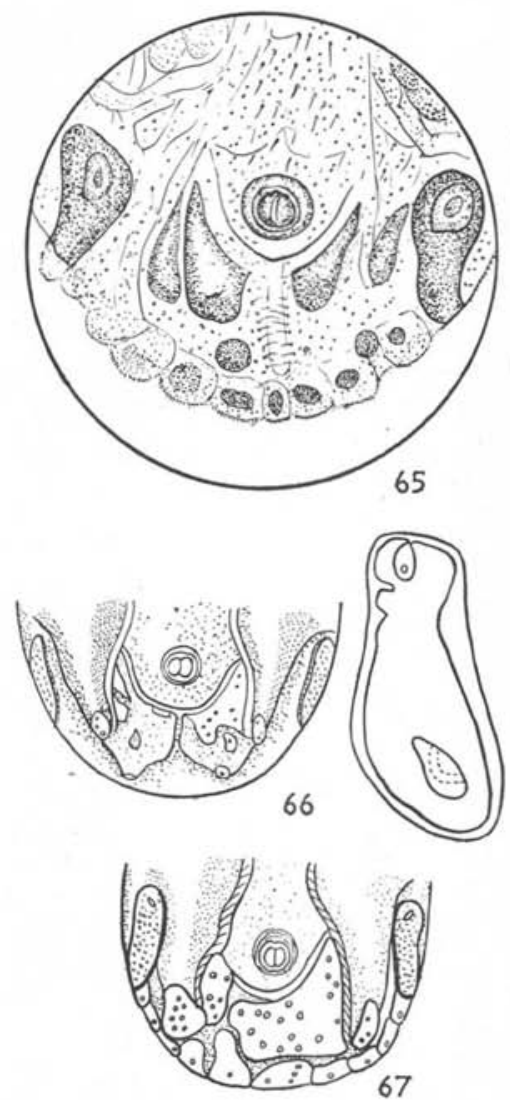

Fic. 65. - Hyalomma sp. Asymétrie des écussons. (D'après Sharif) ; Fic. 66. - Hyalomma excavatum. Asymétrie des écussons. (D'après Starkoff); Fig. 67. - Hyalomma hussaini. Asymétrie des écussons. (D'après Sharif).

II. Fusion de deux écussons :

Hyalomma sp. Afrique du Nord. Sénevet 1922 (fig. 69).

Les écussons anaux sont soudés en arrière de l'anus.

Hyalomma sp. Indes. Sharif 1930 (fig. 70).

Les écussons anaux sont soudés ici en avant de l'anus qui est rejeté en arrière.

Hyalomma impressum transiens Schulze 1927. Mozambique. T. Santos Dias 1947.

Fusion de l'écusson anal et de l'écusson adanal droits. 

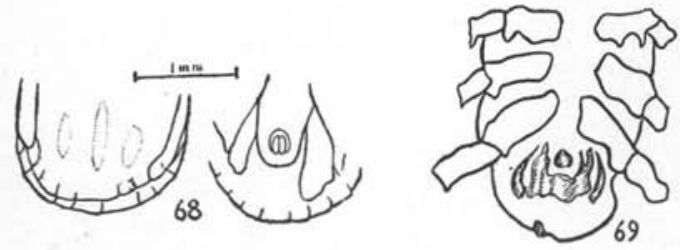

Fic. 68. - Rhipicephalus sanguineus. Asymétrie des écussons. (D'après Nuttall) ; FIG. 69. - Hyalomma sp. Fusion d e s écussons anaux. (D'après Sénevet).

Hyalomma kumari Sharif. Indes. Sharif 1930 (fig. 71).

Fusion des écussons anaux en arrière de l'anus.

Rhipicephalus tricuspis Dönitz 1906. Mozambique. T. Santos Dias 1953 (fig. 72).
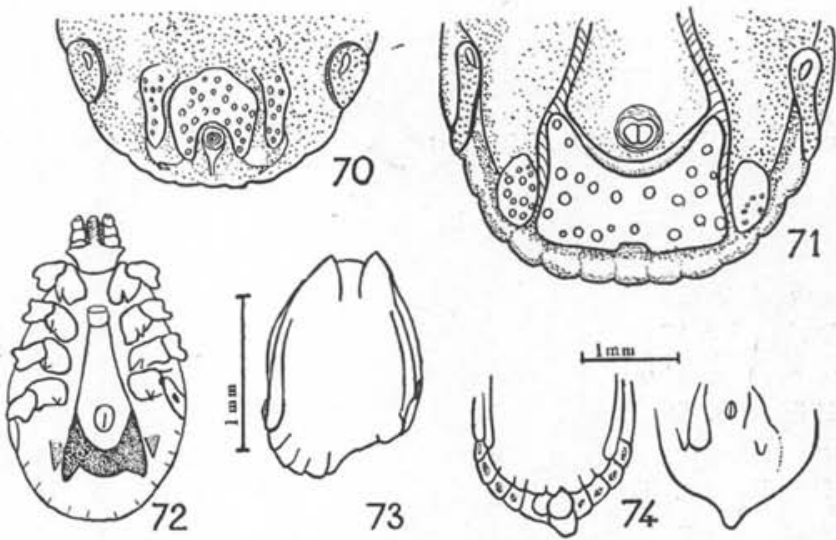

Fig. 70. - Hyalomma sp. Fusion des écussons anaux en avant de l'anus. (D'après Sharif); Fig. 71. - Hyalomma kumari. Fusion des écussons anaux. (D'après Sharif); Fig. 72. Rhipicephalus tricuspis. Fusion des écussons anaux. (D'après Santos Dias); Fig. 73. - Haemaphysalis leachi. Atrophie postérieure et diminution du nombre de festons. (D’après Nuttall); Fıg. 74. - Rhipicephalus longiceps. Anomalie des festons. (D'après Nuttall).

\section{Anomalies des festons}

Les anomalies des festons marginaux sont assez fréquentes. Peu d'auteurs les signalent, car elles n'offrent, en réalité, qu'un intérêt restreint.

Ces anomalies concernent la régularité des festons, leur forme, leur taille, leur nombre; il y a également souvent fusion de deux 
ou plusieurs festons; l'absence d'un certain nombre d'entre eux peut être due à une atrophie de la partie postérieure du corps ; c'est le cas de l'Haemaphysalis leachi (fig. 73) et du Rhipicephalus sanguineus de Nuttall, celui également d'un des Amblyomma hebraeum et de trois Aponoma exornatum de T. Santos Dias.

Les variations sont souvent multiples chez un même exemplaire : il peut y avoir à la fois réduction du nombre de festons et fusion de certains, par exemple. Aussi, pour éviter des redites fastidieuses, avons-nous renoncé à séparer ces anomalies qui peuvent être d'origines diverses.

Nous nous bornons à citer les plus marquantes :

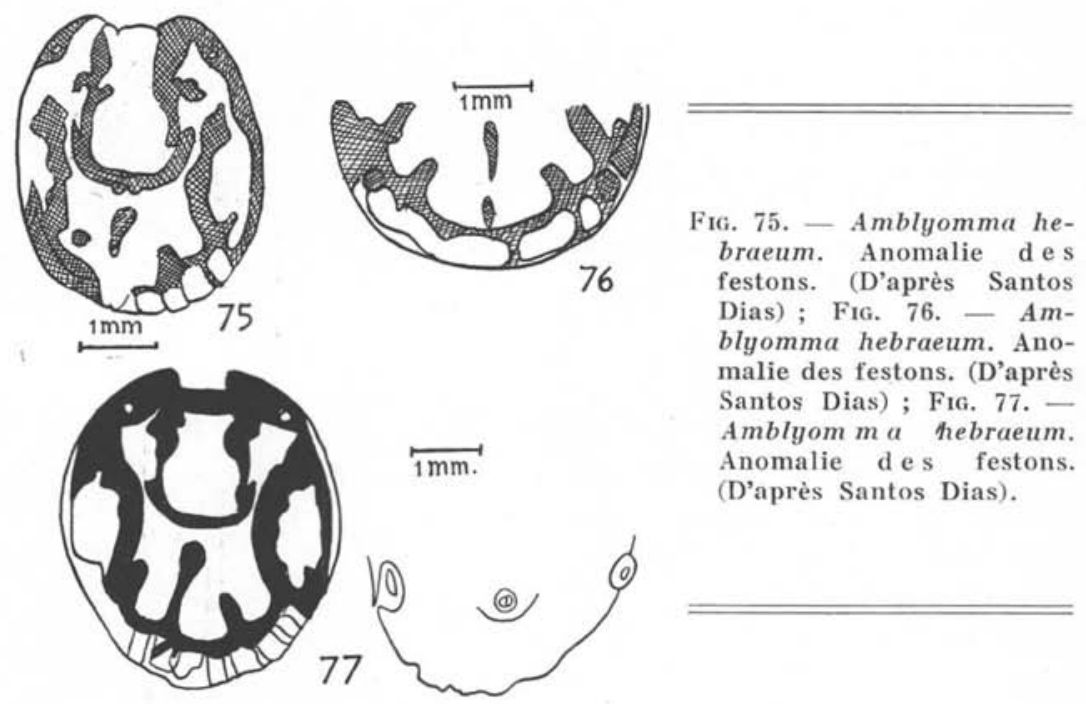

- Nuttall (1915) a décrit un Amblyomma marmoreum dont les festons sont irréguliers et trois fusionnés et un Rhipicephalus longiceps portant sur la pointe caudale deux protubérances (fig. 74).

- T. Santos Dias a donné toute une série de cas observés principalement chez Amblyomma hebraeum (fig. 75, 76 et 77), une vingtaine en tout en trois publications. L'auteur trouve à peu près $2 \%$ d'anomalies chez les tiques qu'il a examinées, et conclut que ces anomalies sont plus fréquentes qu'on ne le croit généralement. En réalité, les anomalies des festons sont loin d'ètre toutes signalées et l'on ne peut guère examiner de collections de tiques sans retrouver de telles irrégularités. Nous-même, en passant en revue les Amblyomma variegatum de la collection du Laboratoire de Parasitologie, avons trouvé une vingtaine de cas du même genre sur 4.000 individus examinés. 


\section{Anomalies diverses}

\section{Eil surnuméraire :}

Neumann, en 1899, signale chez un Hyalomma sp. mâle la présence d'un œil supplémentaire à droite; cet œil est petit et brillant, situé un peu en avant et en-dedans de l'œil normal. Chose curieuse, cette anomalie, qui est historiquement la première signalée chez les Ixodoidea, n'a été retrouvée que très récemment par Pervomaisky (1954), chez un Hyalomma hybride.

\section{Aire poreuse surnuméraire :}

Chez plusieurs femelles d'un élevage de Hyalomma savignyi, FeldmanMuhsam a remarqué la présence d'une aire poreuse supplémentaire sur un des côtés. Cette formation surnuméraire est de même taille que l'aire poreuse normale au-dessus de laquelle elle se trouve située, et en est bien séparée.

\section{Aires poreuses partiellement fusionnées :}

Rhipicephalus simus Koch. Mízambique. T. Santos Dias 1953 (fig. 78).

\section{Stigmate dédoublé :}

Hyalomma savignyi. Palestine. Feldman-Muhsam 1948 (fig. 79).

Un des stigmates comprend deux péritrèmes distincts et inégaux, et deux orifices stigmatiques.

\section{Stigmate surnuméraire :}

Hyalomma anatolicum. Pervomaisky 1954.

Une femelle présente sur la face dorsale, à peu près au même niveau que le stigmate ventral, un stigmate surnuméraire avec deux orifices stigmatiques.

Ces diverses anomalies, fort rares, sont probablement d'origine embryonnaire.

\section{Position anormale de l'anus :}

Nous avons déjà signalé chez le Hyalomma de Sharif (1930) que la fusion des écussons en avant de l'anus avait entraîné un déplacement de cet organe vers l'arrière.

G. G. Robinson (1943) décrit un mâle d'Ornithodorus moubata dont l'anus est rejeté à l'extrémité postérieure ; il n'y a pas de sillon pré-anal ni d'aires discales; les sillons coxaux sont réunis en arrière, formant un demi-cercle derrière l'orifice génital (fig. 80). 


\section{Formations chitineuses anormales :}

Delpy (1936) décrit chez un Hyalomma asiaticum Schulze 1935, une épine chitineuse prolongeant la scapula gauche jusqu'à la hauteur du deuxième article du palpe (fig. 81).

Pavlovsky (1940) montre, chez une femelle d'Ornithodorus tartakovsky, une plaque arrondie située au-dessus et à droite de l'anus, ayant une structure différente de celle de la chitine environnante.

FIG. 78. - Rhipicephalus simus. Fusion partielle des aires poreuses. (D'apr. Santos Dias) ; FiG. 79. Hyalomma savignyi. Stigmate en partie dédoublé. (D'après Feldman - Muhsam) ; FIG. 80. - Ornithodorus moubata. Anus rejeté très en arrière ; sillons coxaux fusionnés. (D'après G. Robinson) ; FIG. 81. - Hyalomma asiaticum. Le bord gauche de la scapula forme une épine. (D'après Delpy).
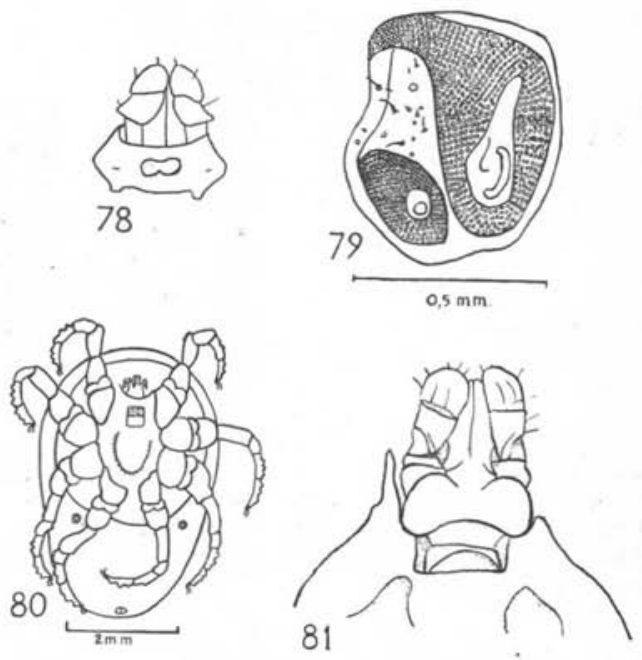

Alfeief (1948) décrit diverses malformations chez une femelle de Hyalomma dromedarii Koch 1844 (fig. 82). A l'avant du scutum, un peu à droite et en arrière de l'ouverture de l'organe de Géné, se trouve une excroissance digitiforme chitinisée dont l'extrémité antérieure atteint le niveau de l'aire poreuse droite; sa base triangulaire se confond avec la chitine du scutum.

L'auteur suppose qu'il s'agit d'une évagination partielle de l'organe de Géné, rendue permanente par la chitinisation. Cette hypothèse nous paraît assez hasardeuse et nous croyons qu'il faut voir là une simple expansion du scutum, due sans doute à un traumatisme antérieur. Cette explication est d’autant plus plausible que la partie antérieure gauche a subi aussi une mutilation : cing articles de la première patte sont absents et le palpe est incomplet (cf. anomalie des pièces buccales).

Nous-même, dans les collections du Laboratoire de Parasitologie, avons pu examiner une femelle gorgée d'Ixodes ricinus ayant un aspect curieux : le corps est resserré vers le milieu, la partie antérieure se trouvant ainsi plus large que le reste de l'abdomen; le bord postérieur est fortement asymétrique, le côté gauche étant plus court que l'autre. 
Le sillon anal se trouve incliné vers la gauche et sa branche gauche est enfoncée dans une dépression qui va en s'accentuant de l'anus vers le bord. $\mathrm{Si}$ on examine le bord postérieur, on constate que la partie rétrécie est maintenue par une bandelette de chitine dure, qui n'a pu se distendre comme le reste du corps (fig. 83).

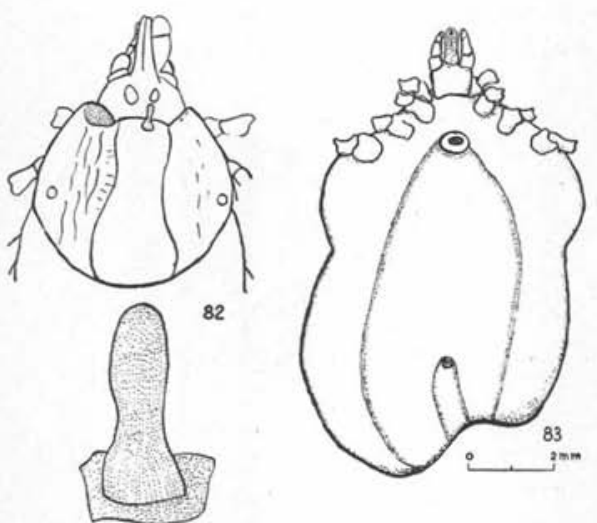

FiG. 82. - Hyalomma dromedarii. Formation chitineuse digitiforme sur le scutum. En bas, la même très agrandie. (D'après Alfeief); Fıg. 83. - Ixodes ricinus. Asymétrie provoquée par une bande de chitine dure. (Original).

Cette formation rappelle un peu l'aspect du Hyalomma de Sharif (1930), que Schulze rattache aux gynandromorphes; chez le Hyalomma, la bandelette de chitine dure se trouve en un endroit normalement recouvert par le scutum du mâle, ce qui rend admissible l'hypothèse du gynandromorphisme, m al g r é une conformation générale bien différente de celles des mosaïques que nous avons déjà rencontrées; chez notre Ixodes, cette bandelette ne correspond, par sa situation, à aucune des ornementations du mâle; nous ne rangerons donc pas cet intéressant spécimen parmi les gynandromorphes. Nous pensons qu'il s'agit simplement d'un durcissement de la chitine au moment de la mue : l'exuvie a pu rester accolée au bord postérieur de la tique et empêcher le développement normal de la cuticule en cet endroit.

\section{Absence d'un œil :}

Amblyomma hebraeum Koch 1844.

$1^{\circ}$ Afrique orientale portugaise. Robinson 1920. L'œil gauche est absent, mais toute la région environnante est détériorée ; il s'agit certainement d'un traumatisme.

$2^{\circ}$ Mozambique. T. Santos Dias 1947. L'absence de l'œil gauche est ici la seule anomalie, il a pu se produire un accident d'origine embryonnaire.

$3^{\circ}$ Mozambique. T. Santos Dias 1952. Absence de l'œil droit. Amblyomma variegatum Fabr. 1794. Mozambique. T. Santos Dias 1948.

Absence de l'œil gauche, sans traces de traumatisme.

Dermacentor marginatus. Russie. Pavlovsky 1940.

L'œil gauche est absent, ainsi que la première patte gauche. L'origine de cette anomalie est donc ici encore traumatique. 


\section{Absence d'un stigmate :}

Les quelques exemples que nous connaissons nous montrent que l'absẹce d'un stigmate est liée à une autre anomalie, dont elle découle probablement.

\section{Hyalomma sp.}

$1^{\circ}$ Robinson 1920. Absence du stigmate droit et atrophie des écussons.

$2^{\circ}$ Sharif 1930. Atrophie du corps à droite, le stigmate manque.

$3^{\circ}$ Sharif 1930. Absence du stigmate droit, des pattes III et IV $d$.

Rhipicephalus simus. Mozambique. T. Santos Dias 1947.

Absence du stigmate droit et atrophie des écussons.

\section{Anomalies des pièces buccales. Malformations du capitulum}

\section{Hypostome :}

Cooper et Robinson (1908) signalent chez plusieurs espèces des inégalités dans les files de dents de l'hypostome.

Dermacentor atrosignatus Neumann 1906. Formose. Robinson 1920 (fig. 84).

Le côté gauche de l'hypostome a subi une forte torsion vers la gauche et les dents qu'il porte sont plus réduites qu'à droite. Il y a en outre, sur la partie non denticulée, trois pointes saillantes dirigées vers l'avant. Cette anomalie pourrait s'expliquer par le fait que la tique ait été arrachée de l'hôte au moment du repas précédent, bien que, le plus souvent, dans ce cas, on trouve le rostre brisé.

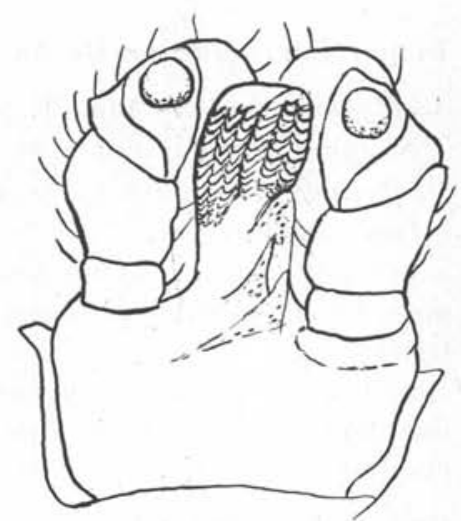

FiG. 84. - Dermacentor atrosignatus. Déviation de l'hypostome ; pointes chitineuses anormales à sa base. (D'après L. Robinson).

\section{Ghélicères :}

Hyalomma dromedarii Koch. Delpy 1936 (fig. 85).

Les gaines des chélicères sont divergentes et tordues; les chélicères sont déplacés en avant de leurs gaines et forment avec elles un angle droit rigide. C'est le seul cas d'ectopie que nous connaissions, il est probablement d'origine traumatique. 

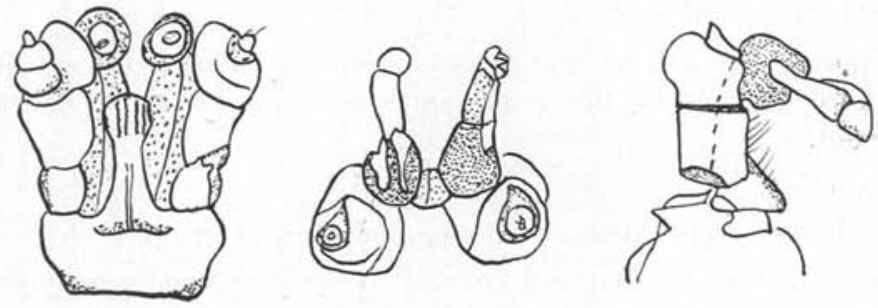

FIG. 85. - Hyalomma dromedarii. Ectopie des chélicères. $A$, vue ventrale; $B$, vue frontale; $C$, vue latérale. (D'après Delpy).

\section{Palpes :}

Haemaphysalis bispinosa. Indes. Warburton et Nuttall 1909.

Les deux palpes sont de taille très différente (non figuré).

Hyalomma dromedarii. Russie. Pavlovsky 1940.

Dédoublement d'un palpe et du rostre; nous décrirons cette intéressante anomalie avec les schizomélies d'appendices.

\section{Disparition d'une partie du capitulum :}

Amblyomma nuttalli Dön. T. Santos Dias 1953.

Nymphe sans capitulum (fig. 86).

Hyalomma detritum Schulze. Russie. Pavlovsky 1940.

Deux exemplaires.

$1^{\circ}$ Le capitulum est totalement absent; la tique est vivante, mais a mué difficilement et les pattes sont molles et plissées, la plaie est cicatrisée.

$2^{\circ}$ Les chélicères et l'hypostome manquent: à leur place se trouve une dépression chitinisée ; on aperçoit par transparence les muscles des chélicères arrachés.

\section{Hyalomma dromedarii.}

$1^{\circ}$ Delpy 1936. La base du capitulum est un peu asymétrique. Le palpe gauche est absent, le droit n'a qu'un article ; les chélicères et leurs gaines n'existent plus, et l'hypostome est légèrement atrophié. En outre, les pattes I $d$, II $d$ et II $g$ sont réduites à deux articles. Aucune patte ne porte de pulvilles.

$2^{\circ}$ Alfeief 1948. Russie (fig. 82). Le palpe gauche présente à sa base un mamelon d'où partent trois articles grêles et peu chitinisés, s'accolant sur une partie de leur trajet à l'hypostome. En outre, l'aire poreuse gauche est plus grande que la droite, et l'épaule gauche, au lieu de se terminer en triangle vers l'avant, forme une surface rectangulaire ponctuée. Le scutum est fortement plissé entre le sillon cervical et l'œil. 
Hyalomma savignyi. Russie. Pavlovsky 1940.

Les palpes et les chélicères manquent, le capitulum est épaissi et déformé, et présente un disque chitineux, l'hypostome est asymétrique. Hyalomma steineri enigkianum Schulze 1950.

$1^{\circ}$ Chez une femelle, deux articles des palpes sont fusionnés et les chélicères manquent complètement.

$2^{\circ}$ Chez un mâle, le capitulum, très rudimentaire, ne porte ni hypostome ni chélicères. Le palpe droit est rudimentaire et le gauche est remplacé par une patte atrophiée (cf. atrophies).

Ixodes ricinus. Zapletal 1957. Tchécoslovaquie.

L'exemplaire que nous avons précédemment classé dans les gynandromorphes ne possède pas de capitulum. Les seules pièces buccales qui restent sont les chélicères, d'ailleurs atrophiés, qui paraissent reliés à la première hanche gauche (fig. 20).

Rhipicephalus sanguineus. Russie. Pavlovsky 1940.

Le palpe gauche est atrophié et le droit absent. Cette anomalie est liée à une malformation tout à fait curieuse de la première patte droite dont nous parlerons plus tard.

Les malformations du capitulum ne présentent pas un grand intérêt, car elles sont manifestement le résultat de traumatismes. Si l'accident a eu lieu au stade même où l'animal a été découvert, il y a une simple cicatrisation ; s'il a eu lieu à un stade précédent, il y a régénération plus ou moins complète. Les lésions causées par l'arrachement d'une Tique au cours du repas peuvent être très variées, ce qui explique la diversité des spécimens décrits.

\section{Anomalies des appendices}

Ces anomalies peuvent être «par excès » (polymélies), «par défaut » (méiomélies), avoir trait à des déplacements d'organes (ectopies) ou des substitutions (hétéromorphoses).

Nous ne traiterons ici ni les ectopies (nous ne connaissons qu'un cas d'ectopie des chélicères, étudié avec les malformations des pièces buccales), ni les hétéromorphoses, jamais signalées chez les Acariens.

Dans les polymélies, on peut avoir des appendices ou segments d'appendices surnuméraires insérés directement sur le corps (somatomélies) ou sur un appendice (mélomélies). Les mélomélies se divisent en hypermélies dans lesquelles les articles supplémentaires sont dans l'axe du membre normal et en schizomélies dans lesquelles les articles supplémentaires forment des ramifications. 
Les méiomélies comprennent les symmélies, atrophies et ectromélies.

\section{POLYMELIES}

\section{I) Somatomélies :}

Les formations surnumérairés, déjà assez rares chez les Insectes, font presque totalement défaut chez les Acariens, sauf dans les cas bien particuliers relatifs aux monstruosités doubles et que nous avons déjà signalés. Ce sont d'ailleurs, probablement, les signes persistants d'une monstruosité double originelle ayant en partie disparu au cours de la vie de l'animal.

Cependant, Feldman-Muhsam signale la présence d'une patte hétérotopique chez Hyalomma savignyi (fig. 87) : une Tique d'élevage présente sur la base du capitulum une excroissance ressemblant à une patte atrophiée, formée de deux articles réduits.
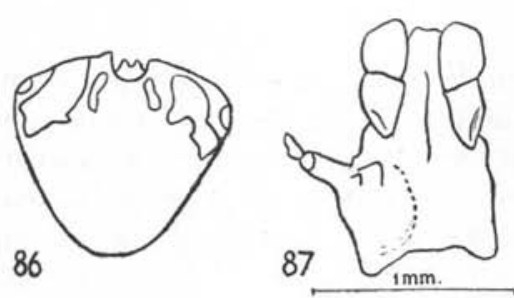

FiG. 86. - Amblyomma nuttalli. Absence presque complète de capitulum. (D'après Santos Dias); Fıg. 87. - Hyalomma savignyi. Patte hétérotopique sur la base du capitulum. (D'après Feldman-Muhsam).

Ce curieux individu représente un cas tout à fait remarquable d'hétérotopie : en effet, chez les Insectes, quand un appendice surnuméraire apparaît, il se trouve inséré très près de l'appendice normal auquel il ressemble, ce qui a fait dire à plusieurs auteurs que ces formations pouvaient s'apparenter aux schizomélies. Ici, la patte supplémentaire est insérée sur la base du capitulum, ce qui exclut l'hypothèse précédente.

\section{II) Mélomélies :}

Nous ne parlerons pas ici des hypermélies dont aucun cas n'a été signalé chez les Acariens.

\section{Schizomélies}

Les schizomélies ou divisions d'appendices sont ici extrêmement rares, alors qu'elles forment un chapitre important dans la tératologie des Insectes. 
Nous suivrons la classification de J. Balazuc qui est simple et complète à la fois. D’après cet auteur, la schizomélie est binaire, ternaire ou complexe, suivant que l'appendice considéré est divisé en deux, trois ou plusieurs branches. Elle est multiple quand elle intéresse plusieurs appendices. Elle peut être combinée à une anomalie d'un autre genre siégeant sur le même membre. Elle peut, enfin, ètre associée à une anomalie différente siégeant sur une autre partie du corps.

\section{Schizomélies binaires.}

L'appendice simple au moins à sa base ou tronc primaire se divise en deux branches secondaires qui peuvent être égales (homodynames) ou inégales (hétérodynames). La bifurcation se fait dans un même plan qui peut être n'importe lequel des plans contenant le trone primaire.

Les branches secondaires peuvent être orientées dans le même sens, ou avoir une symétrie semblable à celle des deux pattes d'une même paire.

La branche normale est celle qui forme, avec le tronc primaire, un ensemble se rapprochant de l'appendice normal ; elle peut se rapprocher davantage de l'axe du membre, alors que la branche supplémentaire forme un angle plus grand avec celui-ci ; cette dernière clause n'est pas obligatoire, les secondaires pouvant être à angles égaux avec le primaire, mais c'est le cas le plus fréquent. Dans les cas d'hétérodynamisme, la distinction entre branche normale et supplémentaire est beaucoup plus facile.

\section{Dermacentor marginatus. Tchécoslovaquie. Cerny 1957.}

La hanche de la première patte droite, élargie, porte deux pattes formées chacune de trois articles courts et trapus, ne portant ni griffes, ni pulvilles (fig. 91).

Hyalomma sp. Indes. Nuttall 1914 (fig. 88).

La deuxième patte droite est bifurquée à partir du trochanter; celui-ci, élargi dans sa partie distale, comporte deux surfaces articulaires supportant chacune quatre articles. La branche normale est dirigée vers l'arrière; la branche supplémentaire se dirige vers l'avant et est d'un tiers environ plus petite que l'autre; à part leur taille réduite, les articles sont normaux et le tarse se termine par des griffes bien développées.

Ornithodorus neerensis. Russie. Pavlovsky 1940 (fig. 89, B).

La première patte gauche possède quatre articles normaux; le protarse s'élargit distalement pour donner deux formations différentes: 
d'une part, se dirigeant vers l'arrière, un tarse normalement articulé et complet, d'autre part, orienté vers l'avant, une expansion longue et épaisse, chitinisée, non articulée, et arrondie à son extrémité.
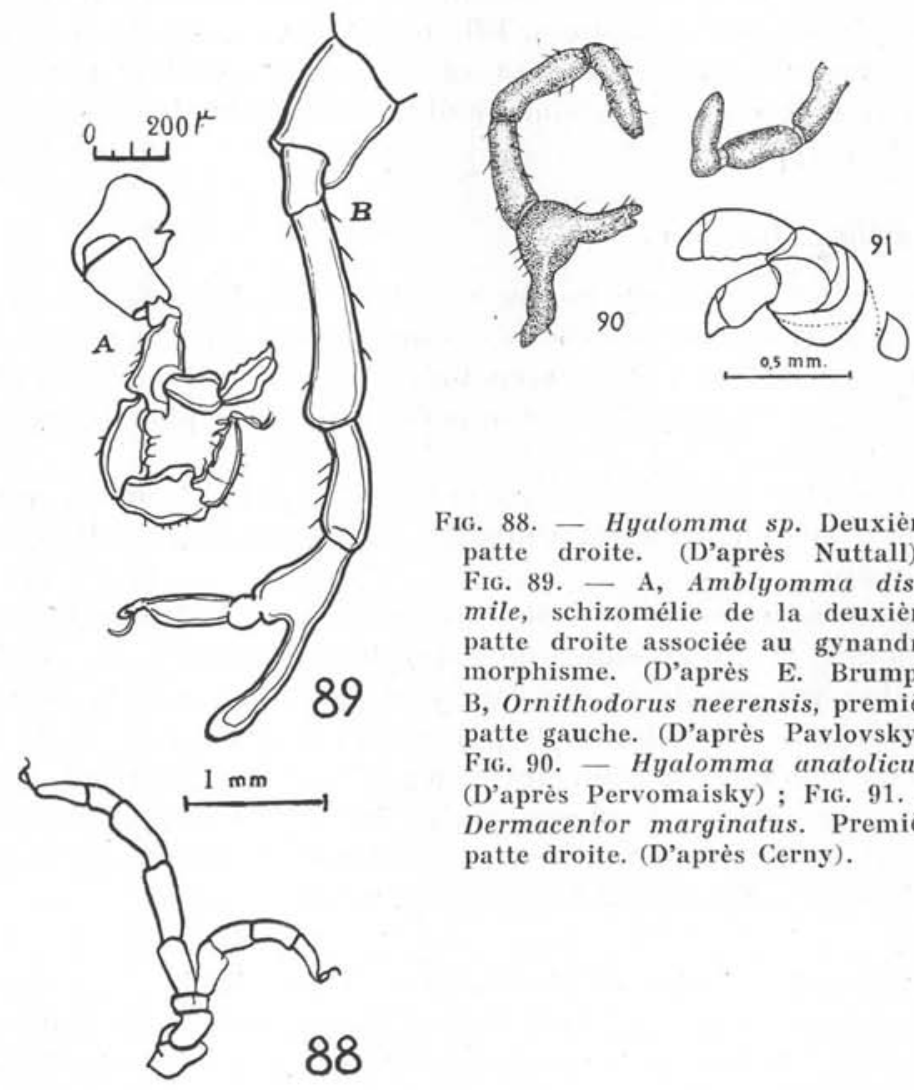

Fig. 88. - Hyalomma sp. Deuxième patte droite. (D’après Nuttall) ; Fig. 89. - A, Amblyomma dissimile, schizomélie de la deuxième patte droite associée au gynandromorphisme. (D’après E. Brumpt). $\mathrm{B}$, Ornithodorus neerensis, première patte gauche. (D'après Pavlovsky); FIG. 90. - Hyalomma anatolicum. (D'après Pervomaisky) ; FIG. 91. Dermacentor marginatus. Première patte droite. (D'après Cerny).

\section{Schizomélies ternaires.}

Rappelons ici les deux lois de Bateson sur la symétrie des appendices surnuméraires :

I. Les axes de l'appendice normal et des deux appendices supplémentaires sont dans un même plan (l'un de ces appendices est plus rapproché que l'autre de l'axe de l'appendice normal).

II. Le plus proche des deux appendices supplémentaires est en structure et en position formé comme l'image de l'appendice normal dans un miroir-plan placé entre l'appendice normal et le plus 
proche, à angle droit du plan des trois axes; et l'appendice le plus éloigné est l'image du plus proche dans un miroir-plan similairement placé entre les deux appendices surnuméraires.

Balazuc donne un schéma de la structure de tels appendices (fig. 92). Les axes longitudinaux du système passent tous par un plan $\mathrm{P}$ passant par $\mathrm{AB}$ (trone primaire). $\mathrm{AB}$ se divise en deux branches secondaires : $\mathrm{BC}$, ou secondaire simple, ne fait qu'un angle léger avec l'axe de $\mathrm{AB}$ et est généralement considéré comme le membre normal ; $\mathrm{BD}$, ou secondaire double, se divise à un niveau variable en deux branches tertiaires, DE et DF, BD révèle toujours une structure double ; il peut occuper la presque totalité de l'appendice supplémentaire ou, au contraire, être réduit à zéro, ce qui

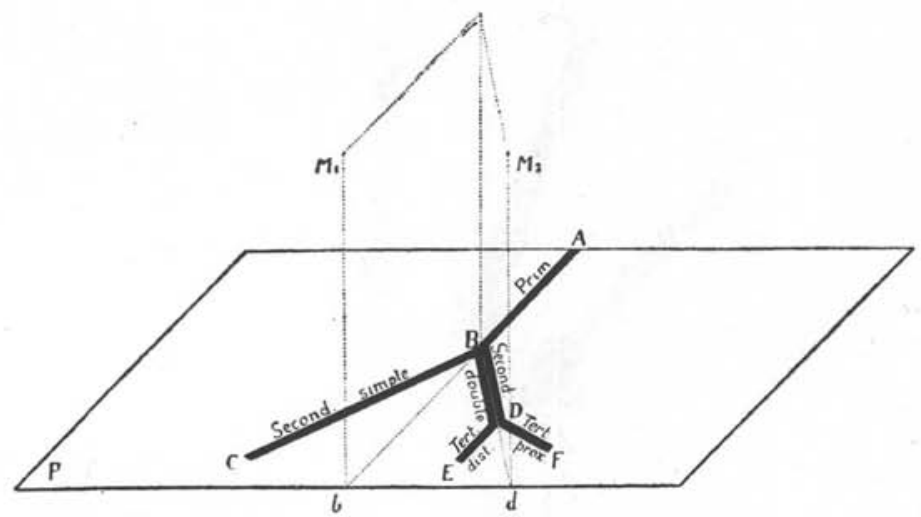

Fıi. 92. - Schéma des schizomélies ternaires.

(D’après Balazuc).

correspond à un aspect typiquement trifurqué du membre, dont les trois branches s'insèrent alors au même niveau. L'angle $A B D$ étant à peu près égal à $\mathrm{ABC}$, l'angle $\mathrm{CBD}$ est le plus petit. $\mathrm{DE}$ sera considéré comme la branche distale du tertiaire et DF comme la branche proximale (par rapport à $\mathrm{AB}$ ). $\mathrm{Si} \mathrm{BDE}$ et $\mathrm{BDF}=\mathrm{BC}$, la schizomélie est homodyname ; si les tertiaires, égaux entre eux, sont plus petits que $\mathrm{BC}$, elle est hétérodyname.

L'orientation des éléments supplémentaires dépend de celle de $\mathrm{AB}$ qui est fixe et du plan $\mathrm{P}$ quelconque. Il y a une relation de symétrie entre les extrémités des trois branches que Bateson représente par un appareil ainsi conçu (fig. 93) :

Un tibia de Coléoptère, avec son tarse correspondant à un membre normal droit (ND), est fixe par rapport au socle de l'appareil ; 
une partie mobile, portant les membres surnuméraires gauche et droit (SG et SD), peut tourner autour de l'axe de ND. Les trois membres portent à leur base des roues dentées engrenées entre elles, et ils sont orientés de façon à ce que deux membres voisins soient symétriques par rapport au plan bisecteur de leurs axes. La rotation du bloc provoque une rotation dans le même sens de SG et dans le sens contraire de SD, ce qui fait que SG tourne deux fois autour de son axe pour une révolution complète, tandis que SD reste parallèle à ND. Pour rendre plus visibles leurs positions respectives, les membres sont peints en noir sur leur face antérieure

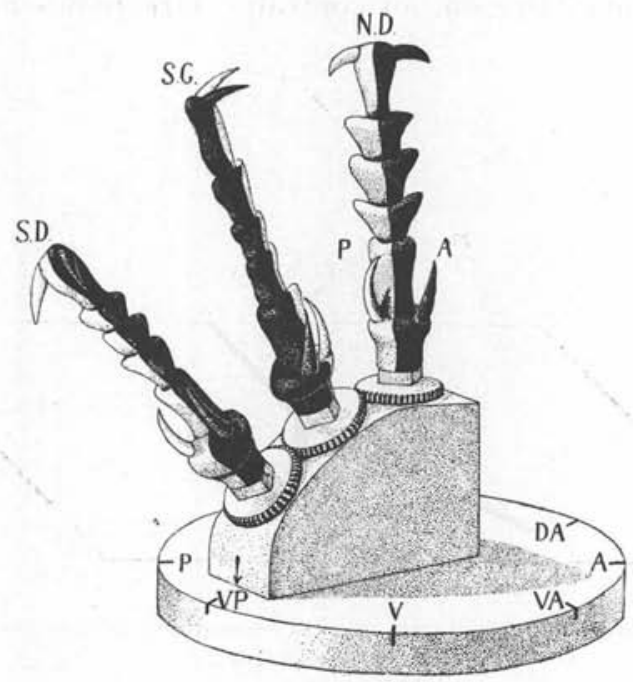

FiG. 93. - Appareil de Bateson illustrant les lois de symétrie des schizomélies ternaires. (D'après Bateson).

et en blanc sur leur face postérieure. Sur le socle se trouvent des repères correspondant aux directions ventrale, dorsale, antérieure, postérieure. Quand le bloc est en direction dorsale ou ventrale, les faces antérieures sont toutes dans le même plan.

Bateson illustre encore les relations de symétrie par un diagramme représentant les positions respectives des trois appendices vus en coupe.

Hyalomma sp. Indes. Sharif 1930 (fig. 94, B).

La quatrième patte gauche, dont les cinq premiers articles sont normaux, se termine par un tarse cylindrique et tronqué distalement 
portant trois pulvilles munis chacun de deux griffes, tous trois de taille normale; il $y$ a en outre une touffe de soies supplémentaires à la base du pulville central.

Dermacentor niveus Neum. 1897. Caucase. Olenev 1931 (fig. 94, A).

La quatrième patte gauche est bifurquée à partir du fémur ; celui-ci s'élargit et porte deux tibias, le tibia antérieur se continue par

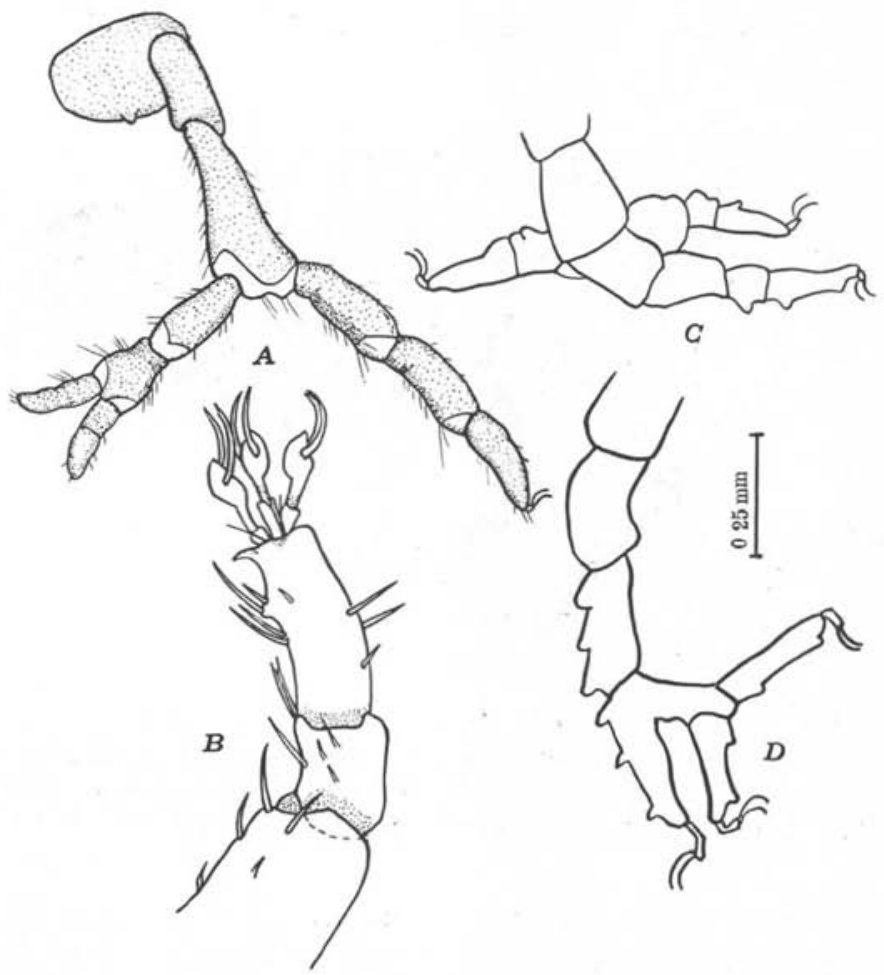

Fig. 94. - A, Dermacentor niveus, $4^{\text {E }}$ patte gauche : fémur portant deux tibias; protarse postérieur divisé en deux tarses égaux. (D’après Olenev). - B, Hyalomma sp., $4^{\circ}$ patte gauche terminée par trois pulvilles et trois griffes. (D'après Sharif). C, Ornithodorus moubata, trifurcation du tibia de la $2^{\text {e }}$ patte droite. (D'après G. Robinson). - D, Ornithodorus moubata, $3^{\text {e }}$ patte gauche : protarse bifurqué, tarse normal également bifurqué.

deux articles normaux, l'ensemble formant la branche normale. Le tibia postérieur, un peu plus petit que l'autre, se continue par un protarse qui s'élargit à son tour pour donner naissance à deux tarses de même taille et ne portant ni griffes ni pulvilles. 
Ornithodorus moubata (Murray 1877) G. G. Robinson 1944. Deux exemplaires.

Chez le premier, qui est une nymphe au $3^{\text {r }}$ stade (fig. 94, C), le tibia de la seconde patte droite porte deux protarses et deux tarses supplémentaires, formant deux membres de taille légèrement réduite, mais normaux. La branche normale conserve son orientation, l'une des branches supplémentaires est dirigée vers l'avant, l'autre vers l'arrière, chacune formant un angle droit avec le fémur. Ces deux segments sont réunis au niveau de l'articulation fémoro-tibiale et retenus par un ligament.

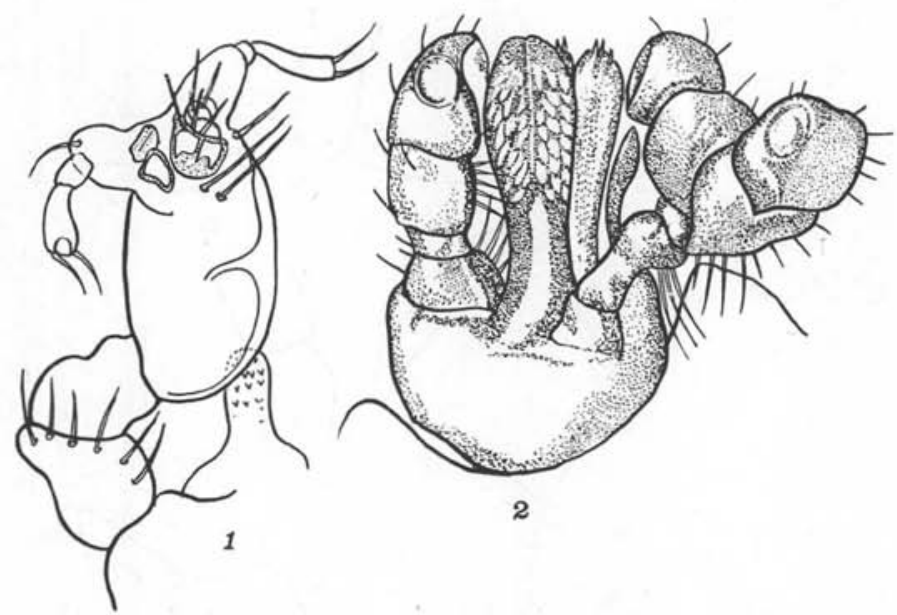

Fig. 95. - 1, Rhipicephalus sanguinens, première patte gauche d'une larve monstrueuse ; 2, Hyalomma dromaderii, schizomélie multiple : palpe gauche et hypostome. (D'après Pavlovsky).

Chez le second exemplaire, qui est une nymphe au quatrième stade, c'est la troisième patte gauche qui présente un tarse bifurqué ; la patte antérieure forme le tarse normal dans le prolongement de la patte, la partie postérieure, plus courte, se divise à son tour, sans doute au niveau de la fausse articulation, en deux branches inégales, portant chacune pulvilles et griffes (fig. 94, D).

\section{Schizomélies multiples.}

Hyalomma dromedarii Koch 1844. Russie. Pavlovsky 1940.

Il s'agit ici d'un cas tout à fait intéressant et unique chez les Ixodoidea de dédoublement des pièces buccales (fig. 95, 2).

Le palpe droit et l'hypostome sont normaux, mais à côté de ce dernier se trouve un deuxième hypostome d'un tiers environ plus petit, 
dont la face interne est dirigée vers la gauche, à la suite d'une torsion de $90^{\circ}$; la face dorsale est restée à peu près parallèle au chélicère gauche, mais elle s'incurve latéralement dans sa partie supérieure.

Les dents sont petites, disposées sur trois files de chaque côté de la suture médiane bien visible.

Le palpe gauche, dont le premier article est long et élargi distalement, est bifurqué au niveau du deuxième article, et donne deux branches à peu près égales, situées dans le prolongement l'une de l'autre, et présentant une torsion de $90^{\circ}$ environ, par rapport au palpe normal.

Les soies qui se trouvent normalement sur la face interne du deuxième segment se trouvent sur les deux branches.

La base de cet appendice est située plus ventralement que de coutume, par suite de la présence de l'hypostome supplémentaire.

\section{Schizomélies associées.}

Amblyomma dissimile. Gynandromorphe décrit par E. Brumpt en 1934 (fig. 89, A).

La deuxième patte droite (côté mâle) porte deux articles de petite taille, sans griffes à l'extrémité, greffés sur la face postérieure du fémur. Cette formation surnuméraire pourrait soutenir la thèse de la duplicité embryologique du gynandromorphisme, si elle se représentait avec une certaine fréquence, mais, comme nous ne connaissons que deux cas de ce genre, nous ne pouvons en tirer aucune conclusion.

Haemaphysalis punctata var. cinnabarina. Monstre double au stade larvaire décrit par de Aboim-Inglez en 1942.

Il s'agit du monstre du $n^{\circ} 21$, présentant un élargissement moyen de la partie postérieure du corps, avec deux anus. La première patte droite porte, implanté sur le trochanter et dirigé vers l'arrière, un rudiment de patte supplémentaire, composé de deux articles; le premier est relié au trochanter par un mince pédicule, le deuxième se termine par deux courtes griffes.

Hyalomma hybride (analolicum plumbeum). Russie. Pervomaisky 1950 (fig. 90).

Chez un des hybrides gynandromorphes, on voit une bifurcation de la $2^{e}$ patte droite, et une déformation de la $3^{\circ}$, qui semblent plus résulter d'un traumatisme que d'une anomalie embryonnaire.

Rhipicephalus sanguineus Latr. Larve monstrueuse décrite par Pavlovsky en 1940 (fig. 95, 1).

Le palpe droit est absent ; la première patte droite est située plus haut que son homologue et présente un aspect tout à fait curieux : la hanche est absente ; au trochanter, court et large, succède un fémur assez large qui supporte, au lieu de tibia, une grosse formation ovoïde, présentant à son extrémité distale deux tarses portant des griffes dirigées respectivement vers la droite et vers l'avant. Sur la base de chaque tarse se trouve un organe de Haller, situé en un lieu tout à fait anormal, puis- 
qu'il est habituellement tout près de l'ongle. Celui de droite est petit et irrégulier, et est surmonté d'un épaississement chitineux, portant deux fines soies. Celui de gauche est plus grand et divisé en deux parties égales par une bandelette chitineuse ; à sa base se trouvent trois grandes soies; son bord antérieur porte trois soies plus fines et plus courtes; il est également surmonté d'une petite plaque chitineuse ovalaire, portant deux soies.

S'agit-il ici d'un traumatisme subi à la sortie de l'œuf ? C'est peu probable, car il y aurait eu à ce moment une simple cicatrisation. Nous paraissons donc bien être en présence d'une malformation embryonnaire.

\section{Théories sur la production des schizomélies}

Pendant longtemps, on s'est borné à voir dans les schizomélies un aspect de la variation sans chercher à en connaitre les causes. Celles-ci peuvent être diverses. Nous savons que, chez les Insectes tout au moins, ces monstruosités peuvent avoir pour origine des mutations ou des fusions d'œufs ou être provoquées par des agents extérieurs.

\section{Mutations.}

Chez les Coléoptères, nous connaissons la mutation V. grube, isolée par Arendsen Hein (1924) chez Tenebrio molitor, puis étudiée par Ferweda (1928), où les antennes sont fréquemment bifurquées ; chez les Diptères, la mutation reduplication de Hoge (1915), qui donne des schizomélies binaires, ternaires ou complexes obéissant aux lois de Bateson. Elle est liée au sexe et semi-léthale. On arrive à multiplier par six le pourcentage d'anomalies en faisant agir le froid.

Chez les Tiques, les schizomélies sont beaucoup trop rares pour avoir une origine de ce genre.

\section{MONSTRUOSITÉS DOUBLES.}

Pour certains auteurs, comme Geoffroy Saint-Hilaire ou Dallas, les schizomélies seraient le vestige d'une monstruosité double originelle, ayant en grande partie disparu au cours de la vie embryonnaire ou larvaire. Cette conception, réfutée ensuite pendant longtemps par la plupart des tératologistes, connut une nouvelle faveur à la suite des travaux de Cappe de Baillon. Cet auteur rencontre en effet beaucoup de schizomélies parmi ses monstres doubles, mais elles ont un aspect particulier. Elles sont le plus souvent sur des appendices surnuméraires et résultent de la concrescence de deux pattes voisines. Ce sont en somme des fusions partielles plutôt que des divisions d'organes. L'auteur pense que certaines 
des schizomélies décrites peuvent avoir une origine diplogénétique, mais se garde bien de conclure que toutes les formations d'organes ont une telle origine. C'est ce qu'a fait à tort Lengerken (1928).

\section{Agents extérieurs.}

Gadean de Kerville (1898) est le premier à prévoir la possibilité de reproduire expérimentalement des bifurcations d'appendices.

Tornier (1900) donne une étude théorique de la production des schizoméfies. Sous le nom de bimélie, il nous montre l'apparition d'un ou deux appendices supplémentaires, ce qui correspond à nos schizomélies binaires et ternaires. Dans le premier cas, le traumatisme provoque par section incomplète de l'appendice la formation d'une surface cruentée d'où partira le membre supplémentaire. Dans le deuxième cas, la section plus large présentera deux surfaces cruentées qui régénéreront toutes les deux, donnant un membre partiellement fusionné.

Sous le nom de trimélie, il invoque l'action à distance du traumatisme ; il y aurait deux lésions, l'une directe par section incomplète, l'autre à distance par fracture. Chaque partie lésée régénérerait un membre supplémentaire.

La première théorie nous parait plus satisfaisante que la seconde qui est plus compliquée et peu plausible.

La théorie de Przibram (Bruch-Dreifachbildung) repose aussi sur l'action à distance d'un choc provoquant une fracture à deux surfaces cruentées, régénérant deux membres supplémentaires de la même façon que dans la bimélie de Tornier. Cette théorie a l'avantage de rendre compte de la direction de l'agent vulnérant.

\section{MEIOMELIES}

\section{Symmélies}

Les symmélies ou fusion d'appendices sont très rares chez les Arthropodes. Rappelons l'intéressante symmélie d'une patte-mâchoire et d'une patte ambulatoire chez un Pseudoscorpion : Chelifer cancroides L., décrite par Vachon en 1947. Nous avons chez les Tiques seulement deux cas :

\section{Amblyomma cayennense (F.). Robinson 1920 (fig. 96).}

Les coxa I et II gauches sont fusionnés, l'armature coxale étant réduite à une seule épine conique, épaisse, dirigée presque perpendiculairement à la surface du corps. Les deux pattes sont ensuite bien séparées et normales. 
Haemaphysalis punctata.

Un des monstres doubles de Aboim-Inglez présente une patte postérieure en partie double résultant de la fusion des quatre premiers articles. C'est seulement un cas particulier, résultant d'une monstruosité double.

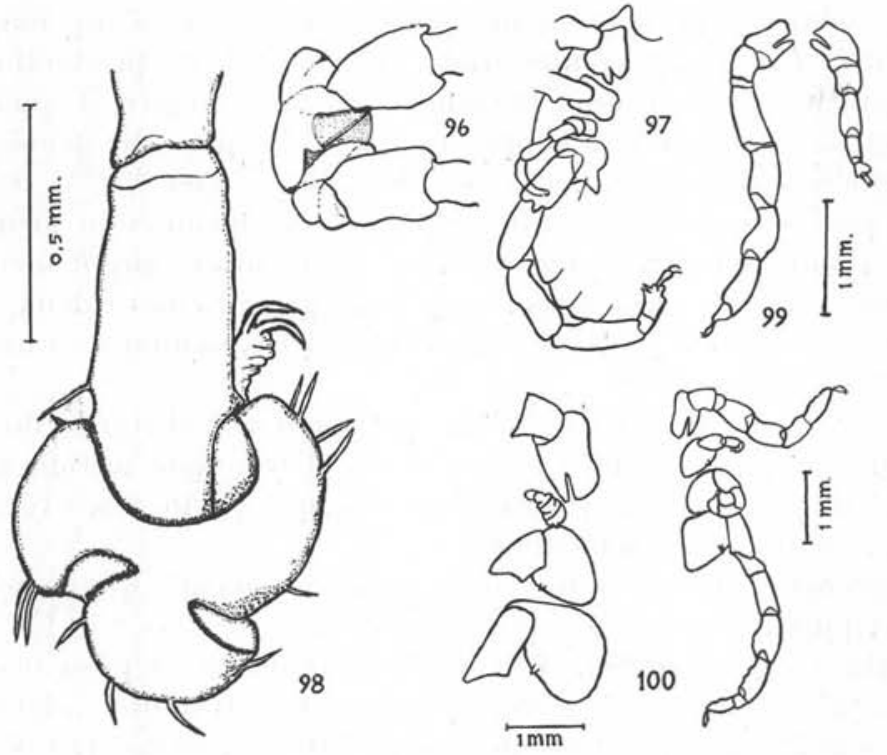

Fig. 96. - Amblyomma cayennense. Fusion des deux premiers coxa. Une seule épine. (D’après L. Robinson) ; Fı́. 97. - Amblyomma hebraeum. Atrophie de la $3^{\circ}$ patte droite. (D'après Santos Dias) ; Fig. 98. - Amblyomma variegatum. Atrophie de la $4^{\circ}$ patte gauche. Les trois derniers articles sont fusionnés. (Original) ; Fic. 99. - Dermacentor marginatus. Première paire de pattes. (D'après Cerny) ; Fı́. 100. - Dermacentor marginatus. Deux exemples d'atrophie. (D'après Cerny).

\section{Atrophies}

Amblyomma hebraeum Koch. Mozambique. Santos Dias 1947 (fig. 97).

La $3^{\circ}$ patte droite est atrophiée : le tarse manque complètement, tous les autres articles sont plus petits que ceux de la patte normale correspondante. L'auteur se demande si cette malformation est congénitale ou accidentelle. Nous verrons plus loin qu'après une mutilation, la patte régénérée est très souvent plus petite que d'habitude, et parfois incomplète.

Amblyomma scaevola Oudemans 1905.

Toutes les pattes du même côté sont atrophiées. 
Amblyomma variegatum Fabr. Collection du Laboratoire de Parasitologie (fig. 98).

La quatrième patte gauche est atrophiée : à la suite du fémur, normal ainsi que les deux premiers articles, se trouve une protubérance arrondie, qui correspond à la fusion des trois derniers articles. On retrouve le pulvillum et les griffes, bien développés, sortant d'un interstice situé entre le bord interne de la protubérance et le fémur, dirigés vers l'arrière. Il s'agit sans doute ici d'un accident au moment d'une des mues; pour une raison quelconque, la patte n'a pu se développer normalement en sortant de l'exuvie, elle s'est trouvée repliée sur elle-même, et la chitine s'est durcie, transformant cette anomalie en une malformation définitive.

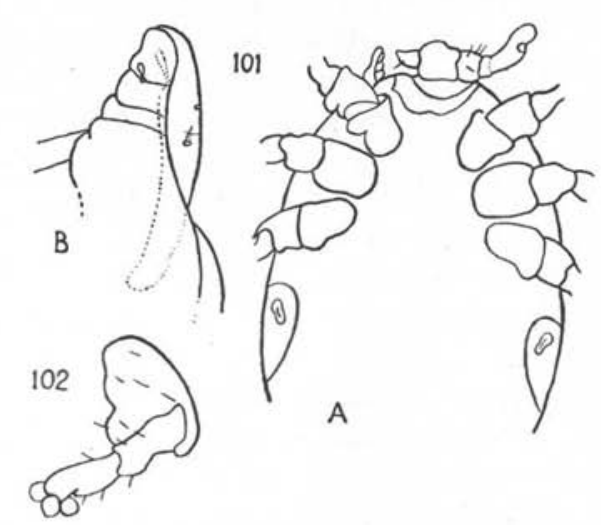

F16. 101. - Hyalomma steineri enigkianum. Anomalies du capitulum et des pattes. B, patte dorsale grossie. (D'après Schulze); Fig. 102. - Amblyomma sublaeve. Absence de la $1^{\text {re }}$ et de la $2^{\circ}$ pattes droites. (D'après Nuttall).

Dermacentor marginatus. Tchécoslovaquie. Cerny 1957.

Trois exemplaires.

$1^{\circ}$ La première paire de pattes est asymétrique : la droite est normale, la gauche est formée de cinq articles plus petits, surtout les derniers (fig. 99).

$2^{\circ}$ La deuxième et la troisième pattes gauches sont très réduites, la deuxième ne porte que trois articles (fig. 100).

$3^{\circ}$ La deuxième patte droite ne possède que quatre articles extrêmement réduits et déformés (fig. 100).

Hyalomma savignyi. Egypte. Feldman-Muhsam 1950.

Plusieurs individus du même lot ont des pattes atrophiées, avec un tarse réduit, sans pulvilles ni griffes. Vingt-deux tiques sur 223 ont au moins une patte anormale. Ce pourcentage étant relativement élevé, l'auteur pense qu'il s'agit d'une malformation héréditaire. 
Hyalomma steineri enigkianum Schulze 1950 (fig. 101).

Le capitulum manque presque totalement ; les chélicères et leurs gaines sont absents. A droite, un appendice portant une soie est probablement le vestige d'un palpe. A gauche, un appendice plus long à trois articles représente, non un palpe, mais la première patte, car il porte un organe de Haller. La première patte droite est remplacée par une formation chitineuse qui part dorsalement: elle est constituée de quatre articles ne portant pas d'organe de Haller.

Les anomalies sont ici multiples, il y a à la fois atrophie, suppression d'organes et hétérotopie. Le traumatisme a dû être assez précoce, probablement pendant la vie embryonnaire.

Ixodes ricinus Neumann 1899.

La quatrième patte droite a des articles plus courts et plus étroits que son homologue; le cinquième article présente une dilatation brusque, le tarse est conique, et ne possède, ni ongles, ni caroncule. L'auteur pense que cette anomalie est la conséquence d'un traumatisme peu grave réparé irrégulièrement.

Rhipicephalus maculatus Neum. Santos Dias 1953.

Atrophie de la troisième patte gauche (fig. 102).

Onithodorus papillipes Birula 1895. Russie. Pavlovsky 1940.

Le tarse d'une des pattes postérieures est de taille réduite, et son extrémité est déformée.

Ornithodorus tartakovskii. Pavlovsky 1940. Russie.

Les tarses de la première paire de pattes sont différents, l'un d'eux ne présente pas de bosses sur la face dorsale.

Ornithodorus verrucosus. Russie. Pavlovsky 1940.

La quatrième paire de pattes est asymétrique, l'une des pattes étant grêle, avec son extrémité déformée.

Sauf le Hyalomma de Schulze, toutes les anomalies ci-dessus sont peu intéressantes. Ce sont des malformations dues à une mutilation aux stades précédents et à une régénération incomplète ou anormale. Elles sont assez fréquentes, mais pas toujours signalées.

\section{Ectromélies}

L'absence d'une ou de plusieurs pattes est une des anomalies les plus fréquemment signalées par les différents auteurs. Nous ne reviendrons pas sur les ectromélies associées à une asymétrie dont nous avons parlé précédemment. Nous nous contenterons de citer quelques cas ne présentant que cette anomalie :

Amblyomma ovale (Koch 1844). Brésil. Beaurepaire-Aragao 1912.

La quatrième patte droite manque complètement. L'espace entre les coxa II et III est augmenté, et la patte III se trouve finalement au niveau 
de l'espace intercoxal III-IV. Le stigmate droit est un peu plus haut que le gauche.

Amblyomma sp. Neumann 1899.

Absence de la première patte gauche. Pas trace de hanche. D'après l'auteur, il ne s'agit pas d'une mutilation ; il existe cependant une petite dépression circulaire avec un bouton chitineux.

Amblyomma sublaeve Neum. Indes. Nuttall 1915 (fig. 103).

Les pattes droites I et II manquent, il y a seulement une petite plaque chitineuse située à la place vacante.

Boophilus calcaratus (Birula 1895). Russie. Pavlovsky 1940.

Absence de la première patte droite.

Boophilus microplus (Can. 1883). Brésil. Souto 1939.

Absence de la deuxième et de la troisième pattes gauches; le coxa de la première patte est élargi et celui de la quatrième est remonté.

Dermacentor marginatus. Russie. Pavlovsky 1940.

Absence de la première patte gauche.

Dermacentor niveus Neum. Olenev 1931.

La quatrième patte gauche est absente, les coxa II et III sont très élargis et déplacés vers le bas.

Dermacentor pictus. Tchécoslovaquie. Cerny 1957.

Deux exemplaires auxquels il manque respectivement la première et la quatrième pattes droites (fig. 104, B et C).

Haemaphysalis chodlokovsky Olenev. Olenev 1931.

Absence de la deuxième patte gauche.

Haemaphysalis concinna (Koch). Russie. Pavlovsky 1940.

Absence de la première patte gauche, avec léger déplacement des autres pattes.

Haemaphysalis punctata černy 1957 (fig. 105).

Absence de la deuxième patte droite.

Hyalomma sp. Indes. Sharif 1930.

Trois exemplaires auxquels il manque une ou deux pattes.

Ixodes ricinus $\mathrm{L}$.

$1^{\circ}$ Olenev 1931 : absence de la première patte gauche.

$2^{\circ}$ Černy 1957 : deux exemplaires auxquels il manque une et deux pattes (fig. $104 \mathrm{~A}, 106$ ).

Rhipicephalus evertsi Neum. Mozambique. Santos Dias 1947 et 1948.

Deux exemplaires auxquels il manque la deuxième patte droite.

Rhipicephalus sanguineus Latr. Indes. Sharif 1930.

$1^{\circ}$ Absence de la première patte droite.

$2^{\circ}$ Absence de la quatrième patte droite, atrophie de la troisième et de la quatrième pattes gauches. 
Ornithodorus papillipes (Birula). Russie. Pavlovsky 1940.

Absence de la quatrième patte droite, il existe une petite fossette à la place du coxa. Pas de déplacement des autres pattes.

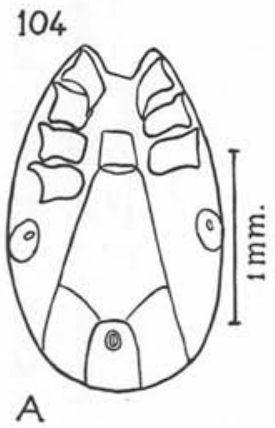

A

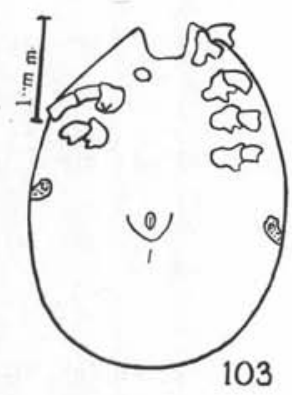

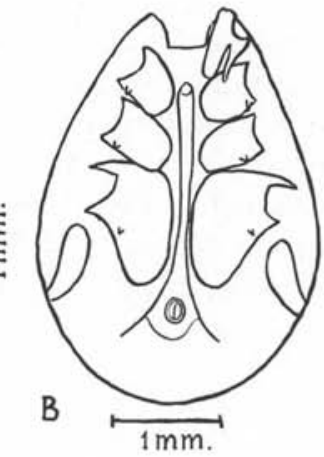

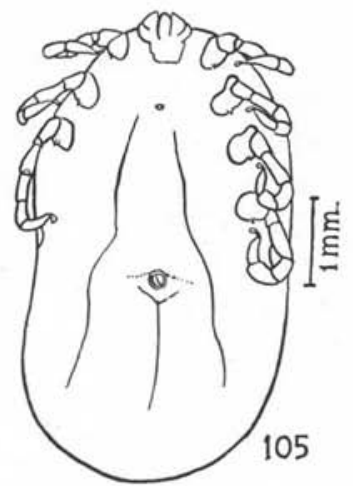

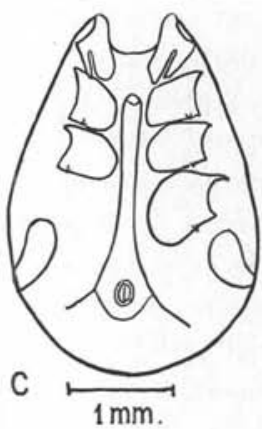

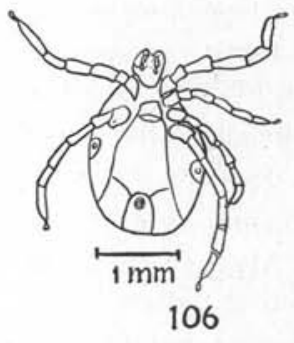

F1G. 103. - Rhipicephalus maculatus. $3^{e}$ patte gauche. (D’après Santos Dias) ; Fic. 104. - A, Ixodes ricinus ; B, et C, Dermacentor pictus : ectromélies. (D'après Cerny) ; - FIG. 105. - Haemaphysalis punctata. Ectromélie. (D’après Cerny) ; Fıg. 106. - Ixodes ricinus. Ectromélie. (D’après Cerny).

Les différents auteurs qui ont signalé des ectromélies séparent nettement ces anomalies de celles, plus légères, qui consistent en une atrophie portant sur la taille des articles ou leur nombre. Les premières seraient des accidents d'origine somatique, alors que les secondes auraient une cause externe mécanique ou physique. Ce point de vue apparaît comme inexact à la lumière d'expériences récentes.

Nous verrons qu'après une mutilation, les pattes ne régénèrent pas toujours et qu'il n'y a parfois aucune trace du membre dis- 
paru. Pervomaisky signale que les ectromélies peuvent être dues à une alimentation sur des plaies séreuses et purulentes, ou à une humidité excessive du milieu au moment de la mue : en humectant tous les jours les récipients contenant des nymphes gorgées de Hyalomma anatolicum, il obtient des malformations et des ectromélies.

Ces faits renforcent considérablement l'hypothèse de l'origine traumatique de telles anomalies; la différence entre atrophies et ectromélies n'est done guère qu'une question de degré.

\section{DEUXIÈME PARTIE}

\section{TÉRATOLOGIE EXPÉRIMENTALE}

Aucune expérience n'ayant été faite jusqu'à ce jour sur les Acariens, sauf en ce qui concerne la régénération des appendices, nous devons nous reporter à ce qui a été fait chez les autres Arthropodes, et principalement chez les Insectes qui ont été de beaucoup les plus étudiés, pour établir des points de comparaison. Beaucoup d'expériences ont été tentées dans un but différent de celui que recherchent les tératologistes, mais elles peuvent cependant présenter un intérêt pour ces derniers par suite des résultats obtenus.

Avec des moyens comparables à ceux qui ont été utilisés chez les Insectes, nous avons tenté de réaliser un certain nombre d'expériences chez les Ixodoidea : les résultats sont souvent bien divergents, ce qui n'a rien de surprenant si l'on considère les différences profondes existant entre ces deux groupes d'Arthropodes.

Il nous a donc paru intéressant de résumer ici les principaux travaux effectués à partir des Insectes pour pouvoir les comparer ensuite avec les résultats de nos propres expériences. Nous n'avons pas la prétention de brosser un tableau complet de l'expérimentation tératogénique chez les Ixodoidea ; ce terrain, totalement inexploré, bien que moins étendu que celui des Insectes, serait encore trop vaste à parcourir, mais nous espérons, en éclaircissant quelques points de ce problème systématiquement ignoré des entomologistes, attirer l'attention de nouveaux chercheurs sur une question qui doit apporter un précieux appui aux connaissances embryologiques et tératologiques actuelles. 
Les principales causes de production d'anomalies sont l'apparition de mutations somatiques ou germinales, l'application d'agents mécaniques, physiques ou chimiques. Nous allons rapidement passer en revue chacune d'elles, en insistant davantage sur les procédés qui ont donné des résultats intéressants dans nos propres cas.

\section{MUTATIONS}

Les mutations n'ont jamais été étudiées chez les Tiques. C'est à peine si l'on connait leur composition chromosomique. Les chromosomes sont au nombre de 11 ou 12 , ils sont généralement courts et épais, et tous plus ou moins semblables, sauf quelquefois les chromosomes sexuels.

Etant donnée l'extrême variabilité de nombreux caractères tels que la taille, l'ornementation, la coloration, seules pourraient être homologuées des mutations portant sur des éléments suffisamment stables tels que le nombre de dents de l'hypostome, la disposition des épines, etc... Nous craignons que ce matériel se prête peu à une étude génétique qui serait pourtant fort utile. En conséquence de l'absence de tout renseignement sur les possibilités de mutations des Tiques, nous ne parlerons pas des mutations produisant des anomalies chez les Insectes. Rappelons, toutefois, l'intéressante mutation reduplication de Hoge (1915), produisant des schizomélies variées chez la Drosophile et dont la proportion augmente sous l'influence du froid.

\section{AGENTS MÉCANIQUES}

\section{Action sur l'œuf}

Ligatures : Seidel (1926) ligature des œufs de Platycnemis pennipes au début de la segmentation et obtient dans un certain nombre de cas un ou deux embryons nains.

Morcellement : Seidel (1929) morcelle à la pince le matériel ovu. laire de Platycnemis pennipes et obtient des monstres doubles dont les composants sont inégaux et encastrés l'un dans l'autre. Krause (1934) obtient également des dédoublements de certaines parties du corps chez Tachycines asynamorus en divisant l'ébauche avec une fine aiguille.

Expériences personnelles : Les œufs d'Ixodidæ sont très délicat:3 à manier, car ils se dessèchent très facilement; il suffit parfois d'éparpiller une ponte pour qu'elle n'éclose pas. 


\section{Morcellement :}

1. Par piqúre: Nous avons tenté de piquer l'embryon par la face dorsale ou ventrale chez Hyalomma sp., avec une aiguille fine, mais tous les œufs sont morts sans poursuivre leur développement. Nous avons donc dû renoncer aux agents mutilants et avons essayé de morceler l'ébauche sans léser la paroi ovulaire dont l'intégrité parait nécessaire à la survie de l'individu.

$2^{\circ}$ Par agitation vigoureuse du tube contenant les œufs.

$3^{\circ}$ Par centrifugation : nous avons soumis à l'action de la cenIrifugeuse électrique, pendant des temps variant de 1 à 5 minutes, divers lots d'œufs de Hyalomma sp. ; l'éclosion s'est toujours produite normalement et nous n'avons constaté aucune anomalie parmi les larves. Les moyens mécaniques ne paraissent donc pas avoir d'action sur les œufs d'Ixodidæ.

\section{Action sur la larve et la nymphe}

\section{A. - Agents non mutilants.}

Gadeau de Kerville (1890) ligature des chrysalides de papillons ou sectionne incomplètement la base des ptérothèques et obtient des adultes à ailes recroquevillées ou réduites.

Tornier (1901) incise les disques imaginaux des élytres chez des larves au dernier stade de Tenebrio molitor et obtient ainsi diverses anomalies de l'élytre chez l'adulte.

Krizenecky (1912) réalise une striction permanente lors de la nymphose en amputant toutes les pattes d'un côté chez les larves de Tenebrio molitor; l'adhérence de la peau au niveau de la cicatrice persiste chez la nymphe et on aboutit à une série de malformations.

Ces expériences ont surtout pour résultats des anomalies des ailes et des élytres, nous ne pouvons donc pas produire l'équivalent chez les Tiques. Nous avons cependant essayé de lier des nymphes gorgées de Hyalomma savignyi avec un cheveu placé en arrière de la première ou de la troisième paire de pattes : si le lien était peu serré, la bête muait normalement; s'il l'était plus, elle mourait sans avoir pu sortir de l'exuvie; dans ce cas, le corps de l'adulte était comprimé, mais paraissait normal, autant que l'on puisse en juger dans ce cas.

Tout récemment, Rehacek (1957) ligature des nymphes gorgées de Dermacentor marginatus et obtient un certain nombre de déformations différentes selon le siège de la striction. Certains de ses 
spécimens ont le bord postérieur échancré, ce qui donne une certaine analogie d'aspect avec les exemplaires cordiformes des monstres doubles, mais l'origine en est essentiellement différente.

Chapman (1913), en écrasant sans effraction des pattes de chenilles de Liparis dispar, obtient des bifurcations avec plus de succès que par amputation.

Balazuc (1948) secoue violemment des larves au dernier stade de Tenebrio molitor, tenues par la patte métathoracique droite au moyen de pinces, et obtient des schizomélies binaires et ternaires au niveau de cette patte ou d'autres pattes, en particulier la métathoracique gauche. La proportion de schizomélies obtenues peut aller jusqu'à $1 / 6$, mais elle est très variable suivant les lots.

Expériences personneiles : Nous avons écrasé des pattes d'Ornithodorus parkeri au troisième stade nymphal sans rompre les téguments, à différents niveaux (trochanter, fémur, tibia, protarse), et nous avons toujours obtenu des régénérats normaux.

\section{B. - Agents mutilants.}

Il s'agit ici de l'amputation d'un appendice suivie ou non de régénération. Celle-ci peut être complète ou incomplète, normale ou double, cette dernière formation étant très rarement obtenue.

Gadeau de Kerville (1890) opère sur différents Coléoptères (Coccinella 7-punctata, Galeruca tanaceti, Tenebrio molitor, Diaperis boleti) et constate qu'il faut au moins une mue pour obtenir un début de régénération, avec un temps suffisant entre l'opération et la mue.

Brindley (1897-98) obtient constamment des Blattes à tarses tétramères au lieu de pentamères après l'amputation.

Tornier (1901) constate que la régénération chez Tenebrio molitor est d'autant plus complète que l'amputation a eu lieu plus longtemps avant la nymphose. Il a observé une fois un régénérat hétéromorphe (antenne terminée en griffe).

Bordage (1905) obtient chez Raphideres scabrosus un tarse monstrueux, coudé, à six articles mal délimités.

Werber (1905) extirpe à la fois l'œil et l'antenne de Tenebrio molitor et obtient une régénération des deux organes.

Megusar (1907) opère sur Tenebrio molitor, Oryctes nasicornis, Rhagium inquisitor et obtient des régénérations commençant dès la mue suivante. Au contraire, avec des Hydrophiles et des Dytiques, la régénération n'a lieu qu'après la mue nymphale ; il observe une fois un régénérat double. D’après Chapman (1913), la régénération complète ne se fait qu'après plusieurs mues chez Liparis dispar; l'auteur note une fois l'apparition de griffes supplémentaires après amputation d'une patte au dernier stade larvaire. 
Egalement chez Liparis dispar, Kopec (1913) obtient une fois l'apparition d'une antenne surnuméraire après ablation d'un œil.

Krizenecky (1913) sectionne longitudinalement le dernier segment de la larve de Tenebrio molitor et obtient quelques régénérats doubles présentant un segment terminal surnuméraire en miniature à côté du segment normal.

Cuénot (1921) détermine des zones actives et négatives pour la régénération des antennes de Carausius morosus : selon le niveau de l'amputation, il $\mathrm{y}$ a régénération ou non, ou hétéromorphose (formation d'une patte à la place d'une antenne).

Przibram (1918) observe la formation lente d'une patte ravisseuse double chez $S$ phodromantis bioculata. Ses essais de régénérations multiples sont peu concluants. Il étudie aussi les régénérations d'antennes chez Oryctes nasicornis (1931).

Abeloos (1933) constate que Timarcha violaceo-nigra (Coléopt.) se comporte comme les Coléoptères aquatiques, la régénération n'ayant lieu qu’à la mue nymphale. Quel que soit le stade larvaire auquel on opère, c'est après la mue nymphale que l'on voit apparaître les appendices régénérés; en outre, les appendices amputés chez la nymphe ne sont pas régénérés chez l'imago. Il n'y a donc qu'une seule période de la vie de l'Insecte, la période pré-nymphale, qui présente une activité régénératrice. Ce phénomène est en opposition avec ce qui se passe chez la plupart des Insectes, les Crustacés et les Tiques, chez lesquels une période d'activité régénératrice apparait quelque temps avant chaque mue.

Bourdon (1937), poursuivant les recherches d'Abeloos, montre que le taux de régénération (c'est-à-dire la longueur de l'appendice régénéré comparée à celle de l'appendice normal) diminue d'autant plus que le niveau de l'amputation est plus proximal ; il est de $80 \%$ si l'amputation est faite à la base de la patte. Il diminue beaucoup si on enlève une partie du thorax et peut devenir nul.

Patay (1937) constate que, chez un Coléoptère de la mème famille (Leptinotarsa decemlineata), la larve peut régénérer au moins partiellement une patte; c'est cependant à la mue nymphale que se manifestent les signes les plus actifs de régénération.

Poisson et Patay (1938) montrent que les résultats sont variables en ce qui concerne la régénération au stade larvaire ; celle-ci dépend de l'évolution du « greffon tarsien » resté à l'intérieur du moignon.

Paulian (1938) obtient parfois une antenne bifurquée en amputant une antenne de Carausius morosus au voisinage des territoires régénérant respectivement une patte et une antenne. Ces bifurcations sont seulement ébauchées et tendent à disparaître aux mues suivantes. 


\section{EXPÉRIENCES SUR LES IXODOIDEA}

Hindle et Cunliffe (1914), étudiant la régénération chez Argas persicus, constatent qu'elle a lieu à tous les stades si l'amputation a été faite assez longtemps avant la mue, et que le membre régénéré est de taille réduite, mais normalement proportionné. Pour chaque stade, il y a une période pendant laquelle aucune régénération ne se produit et cette période est relativement plus longue chez la larve qu'aux autres stades.

Nuttall (1920) complète cette étude par celle de la régénération des pièces buccales, qui se fait dans les mêmes conditions que précédemment. Il étudie également la régénération chez des Ixodidx, A mblyomma hebraeum et Hyalomma sp., et obtient des résultats à peu près identiques, le pouvoir régénérateur paraissant supérieur chez ces derniers, où les pattes et les pièces buccales régénérées sont de taille à peu près normale.

Nous-même (1944-45) avons amputé des Ornithodorus parkeri à tous les stades et avons obtenu les mêmes résultats que pour Argas persicus; nous en avons tiré les conclusions suivantes:

$1^{\circ}$ La régénération est plus complète aux stades âgés qu'aux stades jeunes (surtout le stade larvaire).

$2^{\circ}$ L’appendice régénéré est toujours plus petit que l'appendice normal.

$3^{\circ}$ L'amputation doit être faite assez précocement après le repas pour être suivie de régénération.

$4^{\circ}$ Une amputation tardive retarde la mue suivante, d'autant plus qu'elle a été faite plus longtemps après le repas.

$5^{\circ}$ Une amputation faite très tardivement, entre deux et huit jours avant la mue, ne retarde plus celle-ci, mais ne donne lieu à aucune régénération.

Ces expériences n'ont jamais donné naissance à des régénérations anormales, mais elles aident à comprendre ce qui se passe dans les cas de régénérations doubles que nous étudierons plus loin.

Pour obtenir des schizomélies, nous avons essayé divers procédés :

$1^{\circ}$ section irrégulière des pattes à divers niveaux; nous n'avons jamais eu que des régénérats normaux ;

$2^{\circ}$ maintien d'une plaie béante au moyen d'un petit éclat de bois ; les exemplaires opérés sont tous morts.

Pervomaisky (1954) ampute une ou plusieurs pattes à des nymphes de Hyalomma anatolicum. Quand il respecte les coxa, la régé- 
nération est plus ou moins complète, mais se fait normalement. Quand il enlève les coxa, la régénération est nulle ou faible, et parfois anormale. Il obtient dans un cas une patte bifurquée : la partie antérieure comprend un nombre normal d'articles et des griffes, la partie postérieure plus longue porte un diverticule latéral.

\section{AGENTS PHYSIQUES}

\section{Chaleur}

Action générale.

Nous n'insisterons pas sur les effets de la température : nous savons que, chez les Insectes, la chaleur ou le froid peuvent provoquer des mutations ou les favoriser; ils peuvent également faire apparaitre dans certains cas des malformations des ailes ou des pattes, et aussi des exemples de prothétélie (apparition au stade larvaire de rudiments d'organes imaginaux). Chez les Ixodoidea, les seuls effets constatés sont l'accélération ou le ralentissement de l'évolution de l'animal, avec comme conséquence une abréviation ou une prolongation de la vie, sans apparition d'aucune anomalie.

\section{Action locale : cautérisations.}

Ce procédé a été relativement peu employé jusqu'ici ; il présente cependant une assez grande facilité d'exécution et peut donner de précieux renseignements embryogéniques et tératogéniques.

\section{ACTION SUR L'EEUF}

Seidel (1928) cautérise différentes régions de l'œuf de Platycnemis pennipes (Odonates) et obtient des dédoublements d'organes : la cautérisation du pôle supérieur provoque la duplication plus ou moins marquée des organes céphaliques, allant du dédoublement de la lèvre supérieure à la formation de deux têtes distinctes avec quatre yeux. La cautérisation du pôle inférieur provoque le dédoublement de l'intestin postérieur et des branchies caudales. Enfin, la cautérisation latérale produit un dédoublement partiel du thorax.

Il y a naturellement une grande mortalité à la suite de ces expériences. Nous n'avons pu en pratiquer de cette sorte sur les œufs de Tiques, dont la petitesse et la fragilité rendent l'opération pratiquement impossible. 


\section{ACTION SUR LA LARVE ET LA NYMPHE}

Janda (1913) détruit par cautérisation l'œil et l'antenne de Tenebrio qui régénère partiellement ces deux organes et montre en plus dorsalement un tubercule sétifère que l'auteur assimile à une antenne surnuméraire.

Krizenecky (1913), par la même opération, obtient des excroissances doubles resemblant dans un cas à des antennes.

Cappe de Baillon (1932), par des cautérisations unilatérales ou bilatérales des sclérites au dernier stade larvaire, obtient des bipartitions et des échancrures. Les cautérisations centrales du pronotum donnent une échancrure du bord antérieur, celles du méso- ou du métanotum ne donnent rien.

Nous-mème avons brûlé la hanche de quelques larves de Rhodnius prolixus (Hémipt.), mais tous les exemplaires sont morts avant de muer.

\section{ACTION DES CAUTÉRISATIONS SUR LES IXODOIDEA}

Sergent et Mme Poncet (1943) ont étudié la régénération des pattes de Hyalomma mauritanicum Sénevet. En brûlant une patte ì 200 nymphes gorgées, au niveau de la hanche, ils ont obtenu 38 survivants : la régénération a été nulle chez 4 exemplaires, incomplète chez 19 et complète chez 15 . Au cours d'une seconde expérience, sur 526 nymphes, il y a eu 17 survivants, dont 2 sans régénération et 15 avec régénération incomplète. Les conclusions de ces auteurs sont les suivantes :

$1^{\circ}$ Plus le traumatisme est précoce et moins la régénération se fait.

$2^{\circ}$ L'absence de régénération coïncide avec un retard de la mue.

Ces résultats sont en opposition avec ceux de Hindle et Cunliffe et avec les nôtres qui peuvent se résumer ainsi :

$1^{\circ}$ La régénération a lieu dans tous les cas où l'amputation est suffisamment précoce.

$2^{\circ}$ Le retard de la mue a lieu si le traumatisme est relativement tardif et est accompagné de régénération.

Quelles sont les clauses de ces divergences?

En premier lieu le processus utilisé : une section d'un appendice faite dans les mêmes conditions doit toujours provoquer un traumatisme de même intensité et seul le moment de l'amputation pourra faire varier le résultat final. 
Il est beaucoup plus difficile de doser une cautérisation qui atteint non seulement la hanche, mais les tissus profonds, et détruit ainsi plus ou moins complètement suivant la durée et l'étendue de la brûlure l'ébauche de la patte imaginale. Sergent et Mme Poncet se sont appliqués à brûler « aussi complètement que possible 》 la hanche de deux pattes d'une mème nymphe; la forte mortalité qui a grevé ces expériences montre que ce procédé était trop brutal et, dans ce cas, il est difficile d'établir un rapport entre le retard de la mue et l'absence de régénération.

Le matériel employé doit aussi être mis en cause : nous avons eu des résultats très différents avec les mêmes procédés pour les Ixodidæx et les Argasidæx, ces derniers régénérant plus facilement après brûlure. Après amputation, les résultats sont moins dissemblables, bien que le pouvoir régénérateur paraisse au contraire plus grand chez les Ixodidx (Hindle, 1920).

L'emploi de nymphes de Hyalomma en sommeil hibernal parait de nature à fausser les résultats; la régénération ne doit pas se faire aisément dans cette période de vie ralentie ; c'est peut-être ce qui explique qu'ici un traumatisme plus tardif donne lieu à une régénération plus complète parce qu'il intervient dans la période d'activité précédant la mue. Dans nos expériences, la cautérisation d'une ou deux pattes a toujours amené un retard de la mue, qu'il y ait régénération ou non.

Pervomaisky essaie quelques cautérisations chez des hydrides de Hyalomma : il obtient des régénérations incomplètes ou nulles, et pas de duplications.

\section{Expériences personnelles:}

\section{Expérience sur $O$. parkeri}

Dans une première série d'expériences, nous brûlons nos nymphes avec une pointe mousse rougie, que nous appliquons aussi rapidement que possible sur une des hanches, de façon à faire apparaître une plaque ocracée sur la cuticule, sans former de plaie ouverte; dans ce cas, en effet, les individus ne survivent jamais.

Nous obtenons les résultats suivants en employant ce procédé : sur 16 Tiques, une meurt, deux ne muent pas, quatre ne régénèrent pas la patte atteinte, trois n'ont que des moignons très réduits, une régénère une patte normale et six ont des pattes bifurquées. Nous désignons, par la lettre $\mathrm{B}$, les animaux à pattes bifurquées obtenus par brûlure ; le chiffre correspond à l'ordre chronologique de l'apparition de ces anomalies. 
B 1. - Il s'agit d'une nymphe au quatrième stade, gorgée 48 heures auparavant, à laquelle nous avons brûlé la deuxième patte droite, et atteint en même temps le bord postérieur de la hanche $\mathrm{I}$. La mue s'effectue 50 jours après et nous nous trouvons en présence d'une nymphe avec deux pattes bifurquées (fig. 107).

$1^{\text {re }}$ PATTE DROITE : La branche principale a une direction et une taille normales; du trochanter élargi, surélevé et plus ou moins fusionné avec la hanche, part vers l'arrière un second fémur suivi de trois articles bien conformés, le tarse portant griffes et pulville et mème un organe de Haller ; cette branche secondaire est d'environ $1 / 3$ plus petite que la branche principale.

$2^{*}$ PATTE DRoite : La brûlure ayant été plus profonde qu'à la patte précédente, la régénération est moins complète, la hanche et le trochanter sont réduits ; le fémur se divise dans sa partie proximale en deux branches égales, de direction perpendiculaire à celle de la première patte; la branche interne comporte trois articles petits, mais bien formés ; la branche externe a la même longueur, mais les trois articles, peu chitineux, ont été détériorés, ils sont grêles et plus ou moins fusionnés. Cette patte se dessèche assez rapidement.

Les quatre cas suivants sont des nymphes au cinquième stade gorgées quatre jours avant l'expérience :

B 2. - Cette nymphe mue 17 jours après la brûlure pour donner une femelle et présente une ébauche de bifurcation de la quatrième patte gauche ; la branche principale est petite et grèle ; sur sa face ventrale, on voit, à la base du trochanter, un moignon dirigé perpendiculairement à l'axe de la patte (fig. 109).

B 3. - La femelle issue de la mue 18 jours après la brûlure de la troisième patte gauche ne possède qu'une hanche réduite divisée en deux par une bandelette de cuticule, continuant celle du corps sans trace d'articulation. Si la patte avait repoussé, elle aurait sans doute été double, depuis la hanche (fig. 109).

B 4. - La mue a lieu 20 jours après la brûlure de la première patte gauche qui, chez la femelle, montre une expansion latérale du trochanter sur laquelle s'insère un petit article conique (fig. 109).

B 6. - Au $24^{\circ}$ jour, l'animal mue donnant une femelle dont la deuxième patte gauche est bifurquée à la base du fémur et présente deux branches de taille presque égale, l'une étant un peu plus grêle et sans griffes, mais avec des articles bien formés (fig. 109).

Au cours de l'expérience suivante, nous utilisons des nymphes au troisième stade, gorgées depuis quatre jours. La régénération 
se fait moins bien, elle est nulle dans un cas et incomplète dans trois autres; nous obtenons cependant un mâle à patte bifurquée :

B 5. - La brûlure de la deuxième patte droite donne, après une mue au $23^{\circ}$ jour, un moignon ; par contre, la première patte, qui n'avait pas été atteinte directement, présente une expansion assez importante de l'extrémité du fémur (fig. 109).
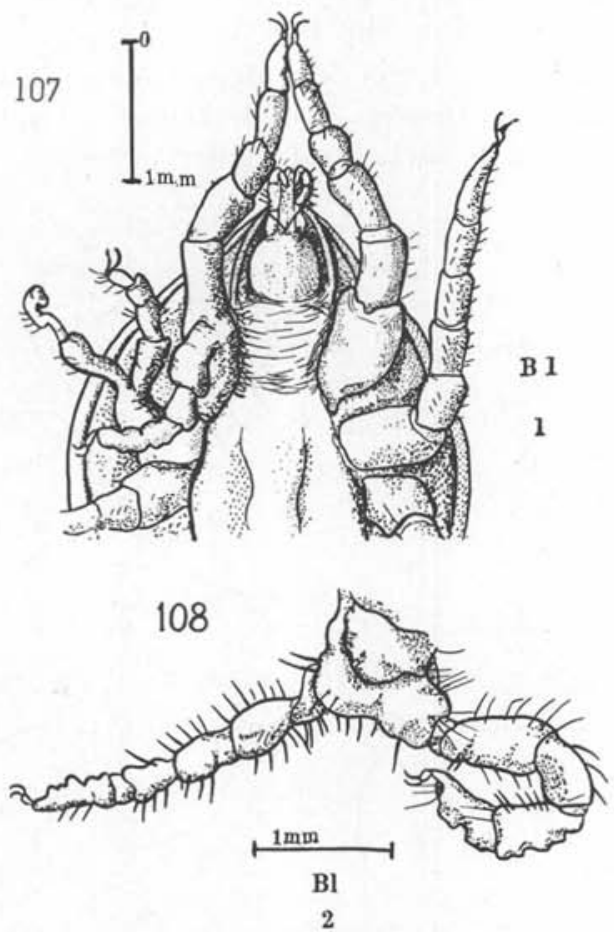

Fig. 107. - Ornithodorus parkeri. Nymphe $\mathrm{B} 1,1^{\text {re }}$ et $2^{\circ}$ pattes droites régénérées après une mue suivant la brûlure; Fig. 108. - Ornithodorus parkeri. Nymphe B 1, $1^{\text {re }}$ patte après deux mues suivant la brûlure.

Nous avons ensuite remplacé la pointe mousse par un fil de nickel-chrome qui a l'avantage de ne pas se déformer et conserve plus longtemps une température élevée; les résultats obtenus sont tout à fait comparables aux précédents. Pour cette expérience, nous avons utilisé des nymphes au quatrième stade nourries depuis quatre jours. Une ne mue pas, une ne présente qu'un moignon, deux régénèrent normalement leurs pattes et cinq montrent des schizomélies. 
B 9. - 16 jours après la brûlure, nous obtenons une femelle dont la deuxième patte droite possède un trochanter élargi sur lequel s'articulent, d'une part, une patte normale, d'autre part, un article unique de forme conique (fig. 109).

B 10. - Un mâle issu de la mue le $18^{\circ}$ jour a sa première patte bifurquée dès la hanche; la partie antérieure est tout à fait normale, la patte postérieure est plus petite, mais porte également des griffes et l'organe de Haller (fig. 109).

B 11. - Une nymphe brûlée à la deuxième patte gauche mue le $19^{\circ}$ jour, et donne une femelle dont le fémur est bifurqué vers le milieu, donnant deux branches sub-égales portant toutes deux des griffes (fig. 109).

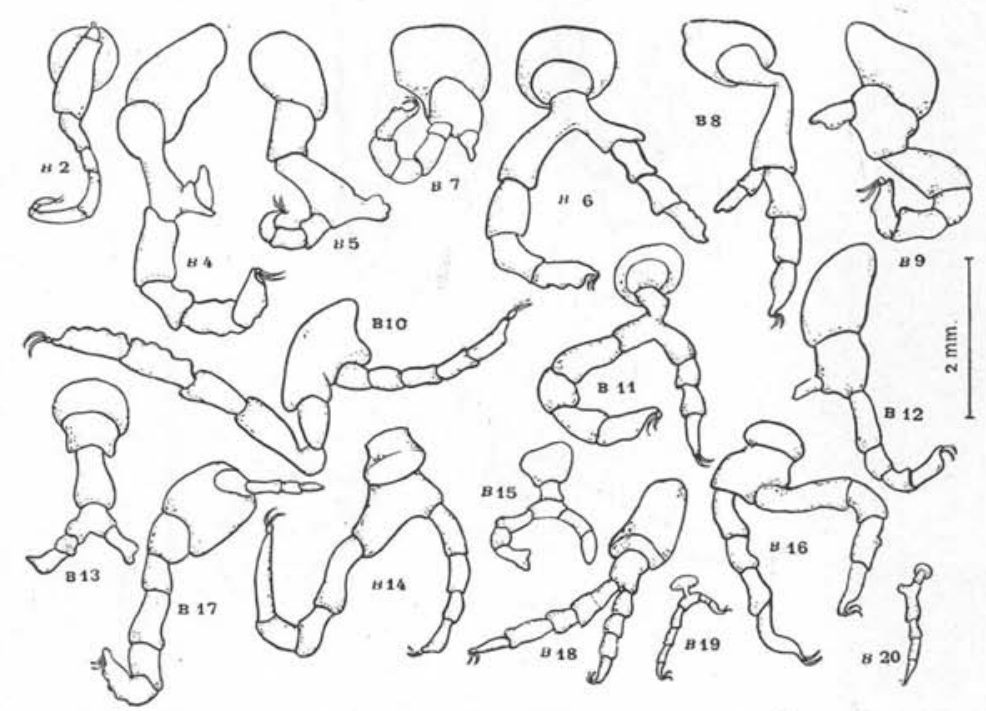

Fıt. 109. - Ornithodorus parkeri. B 2 à B 20, schizomélies obtenues par brûlure sur des nymphes à différents stades.

B 14. - Ce mâle, ayant mué le $22^{\circ}$ jour, a la quatrième patte gauche divisée, à partir du trochanter, en deux branches inégales, mais complètes (fig. 109).

B 18. - La mue n'a eu lieu que le $49^{\circ}$ jour après la brûlure, donnant un mâle dont la première patte droite est, comme dans le cas précédent, divisée en deux branches inégales, partant du trochanter (fig. 109).

Nous avons cherché à perfectionner notre méthode en utilisant une source de chaleur constante et nous avons utilisé la forge du 
micromanipulateur de Browaeys, constituée par un fil de platine chauffé électriquement. Sur 39 individus ainsi opérés au quatrième stade nymphal, 9 meurent, 8 ne muent pas, 2 ne régénèrent pas, 7 n'ont que des moignons, 9 régénèrent normalement, et 4 ont des pattes bifurquées.

B 7. - Une brûlure de la deuxième patte droite est effectuée 9 jours après le repas ; la mue s'ensuit le $21^{\mathrm{e}}$ jour, donnant une nymphe au $5^{\circ}$ stade avec un trochanter élargi portant, outre la patte normale, un petit article mal formé (fig. 109).

B 8. - La nymphe, gorgée 8 jours avant la brûlure, mue 22 jours après ; l'extrémité distale du fémur de la $2^{\circ}$ patte gauche porte, outre les trois articles normaux, deux autres plus petits, mais complets (fig. 109).

B 12. - Une brûlure de la première patte gauche 8 jours après le repas est suivie d'une mue le $27^{\circ}$ jour et donne, chez le mâle, une patte tout à fait comparable à celle de B 7 (fig. 109).

B 13. - A la brûlure de la deuxième patte droite, le lendemain du repas, fait suite une mue le $24^{e}$ jour, donnant une nymphe au $5^{\circ}$ stade ; le tibia est bifurqué et présente, d'un côté, deux articles, de l'autre, un seul; aucune des deux branches ne porte de griffes (fig. 109).

La microforge s'étant révélée d'un usage peu pratique, nous avons finalement utilisé un galvanocautère à fil de platine, à température réglable par un rhéostat. Nous pouvons alors travailler sous la loupe binoculaire qui nous donne un grossissement bien suffisant et rend l'opération beaucoup plus aisée. Les résultats obtenus sont tout à fait comparables aux précédents.

Des nymphes au $5^{\text {e }}$ stade sont soumises à la brûlure d'une ou deux pattes le jour du repas. Deux régénèrent normalement, deux n'ont que des moignons et deux ont des pattes bifurquées.

B 16. - La femelle issue de la mue le $20^{\circ}$ jour présente une bifurcation du trochanter de la troisième patte gauche, donnant naissance à deux branches presque égales et complètes ; seul, le fémur est moitié plus petit d'un côté, les autres articles sont semblables (fig. 109).

B 17. - Après une mue le $23^{\circ}$ jour, le trochanter de la première patte gauche est élargi et bifurqué, donnant en avant une branche normale, en arrière quatre articles très petits, où manquent les griffes (fig. 109).

Dans une nouvelle série d'expériences, nous avons pris des nymphes au deuxième stade afin de pouvoir suivre l'évolution de la 
schizomélie pendant plusieurs mues ; malheureusement, la mortalité a été très élevée. Les nymphes jeunes sont plus fragiles que les autres et la surface brûlée est relativement plus grande. Nous avons cependant obtenu quelques schizomélies. Sur 72 individus, 52 meurent, un ne mue pas, onze ne régénèrent pas, quatre n'ont qu'un moignon, un régénère normalement et trois ont des pattes bifurquées.

B 15. - La brûlure est effectuée sur la hanche de la $4^{\circ}$ patte droite, la mue a lieu le $12^{\circ}$ jour ; la quatrième patte ne régénère pas, mais la troisième est bifurquée au niveau du fémur qui porte d'un côté trois articles, et de l'autre deux; aucun ne possède de griffes (fig. 109).

B 19. - Une nymphe du même lot que B 15 mue 17 jours après la brûlure de la deuxième hanche droite ; cette patte manque, mais la troisième est bifurquée au niveau du trochanter; celui-ci est grêle, il en part deux branches, l'une de quatre articles, l'autre de trois, petits, mais complets, avec des griffes. La patte se dessèche ensuite et tombe (fig. 109).

B 20. - Une nymphe du même lot que B 15 mue 19 jours après la brûlure de la deuxième patte droite qui ne régénère pas; la troisième présente une ébauche de bifurcation du fémur (fig. 109).

Notre dernière expérience porte à nouveau sur des nymphes au quatrième stade, gorgées quatre jours avant la brûlure. Sur huit individus, un meurt, un ne régénère qu'avec un moignon, un régénère normalement et cinq ont des pattes bifurquées.

B 21. - La mue a lieu le $28^{\circ}$ jour, donnant naissance à une nymphe dont la première patte droite est bifurquée à partir de la hanche, donnant deux branches inégales, mais complètes (fig. 110).

B 22. - La mue au $28^{\circ}$ jour après brûlure de la $3^{\circ}$ patte droite donne une nymphe dont la troisième patte manque, alors que la quatrième est bifurquée à la base du fémur ; la branche principale est normale, alors que la branche secondaire ne comporte que deux petits articles (fig. 110).

B 23. - Une brûlure de la quatrième patte droite provoque l'apparition d'une bifurcation de la $3^{\circ}$ patte chez un mâle ; il s'agit d'une expansion de l'extrémité du fémur; la quatrième patte manque (fig. 110).

B 24. - Après une mue au $28^{\circ}$ jour, le mâle présente une bifurcation de la troisième patte droite, la quatrième qui avait été brûlée étant absente. La base du fémur est élargie et porte un article surnuméraire peu développé (fig. 110). 
B 25. - Une mue au $18^{\circ}$ jour nous donne une femelle dont la $2^{\circ}$ patte droite présente un article supplémentaire à la base du fémur tout à fait comparable à B 24 (fig. 110).

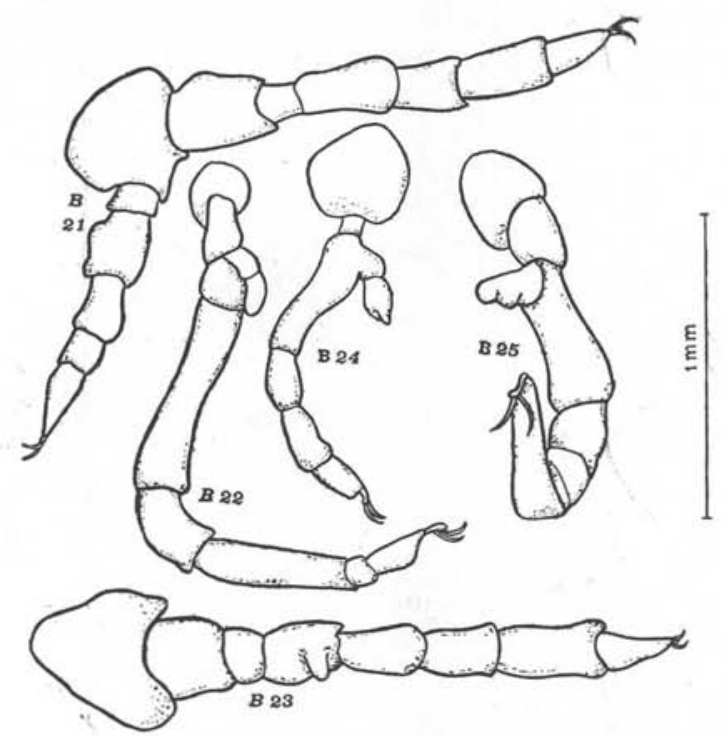

FIG. 110. - Ornithodorus parkeri. B 21 à B 25 , schizomélies obtenues par brûlure sur des nymphes au $4^{\circ}$ stade.

\section{Expériences sur d'autres espèces d'Ornithodores}

\section{Ornithodosus turicata.}

Ces animaux étant très voisins d'O. parkeri, nous espérions avoir des résultats très comparables aux précédents, ce qui s'est trouvé réalisé. Sur six exemplaires au quatrième stade, gorgés depuis deux jours, un n'a pas régénéré, deux n'ont eu que des moignons, et trois ont eu des pattes bifurquées.

B 26. - La femelle issue de la mue le $32^{\circ}$ jour après brûlure de la première patte gauche n'a qu'un moignon à cette place; la deuxième patte a une branche élargie portant en arrière une ébauche d'article et en avant une patte filiforme qui se dessèche et tombe par la suite (fig. 111).

B 27. - Un mâle sorti de la mue le $25^{\circ}$ jour a la hanche de la première patte gauche large et saillante ; en avant, se trouve la patte 
normale plus petite que son homologue, en arrière, on trouve seulement deux articles. La $2^{\circ}$ patte gauche est atrophiée et le tibia est élargi.

B 28. - Une femelle ayant mué le $41^{\circ}$ jour a un aspect très voisin du mâle. La branche antérieure de la $1^{\text {re }}$ patte est normale, la branche postérieure est constituée par trois petits articles (fig. 111).
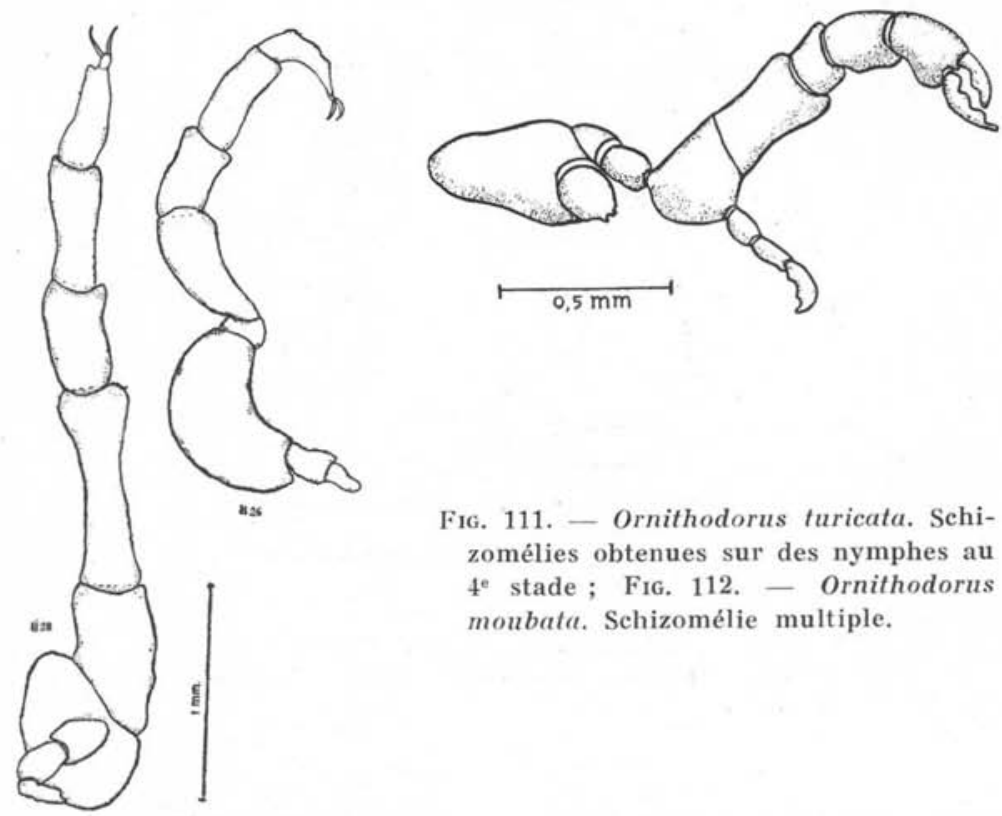

F1G. 111. - Ornithodorus turicata. Sehizomélies obtenues sur des nymphes au $4^{e}$ stade; FIG. 112. - Ornithodorus moubata. Schizomélie multiple.

Ornithodorus moubata, Murray.

Il s'agit ici d'animaux très différents morphologiquement et biologiquement des précédents, il était donc intéressant de savoir si la méthode de brûlure réussissait également sur eux. Sur 20 individus au $3^{\circ}$ stade nymphal gorgés depuis cinq jours, deux sont morts, huit ne régénèrent pas, sept n'ont que des moignons, deux régénèrent normalement et un présente une bifurcation complexe. La première patte gauche a en effet une ébauche de bifurcation de la hanche; le fémur élargi se divise à son tour, portant d'une part trois petits articles, de l'autre trois articles de taille normale, dont le tarse est divisé à l'extrémité distale, formant une sorte de pince sans griffes (fig. 112).

La proportion de schizomélies obtenue est beaucoup plus faible ici ; par contre, c'est notre seul cas de schizomélie complexe. 
Notons en outre que, après la deuxième mue, les exemplaires n'ayant pas régénéré au stade précédent présentent des moignons ou des régénérats normaux.

\section{Persistance de la schizomélie pendant toute la vie de l'Ornithodore}

Dans la plupart des cas, nous avons brûlé des nymphes au troisième, quatrième et cinquième stade, aussi les schizomèles obtenus sont-ils le plus souvent adultes. Il y avait toutefois parmi les exemplaires que nous avons déjà décrits quelques nymphes que nous avons nourries de façon à avoir une nouvelle mue. Nous avons pu ainsi étudier le comportement de nos anomalies à un stade ultérieur; nous donnons ici le résultat de nos observations :

B 1. - Chez la nymphe au sixième stade, la deuxième patte droite qui s'était desséchée n'a pas reparu; il ne reste que la hanche. La première patte est toujours bifurquée; la branche postérieure est maintenant de la même taille que la branche antérieure et seulement un peu plus grêle. La régulation a donc tendu à parfaire la symétrie. Nous avons nourri à nouveau cet intéressant exemplaire

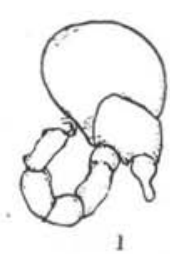

B 7
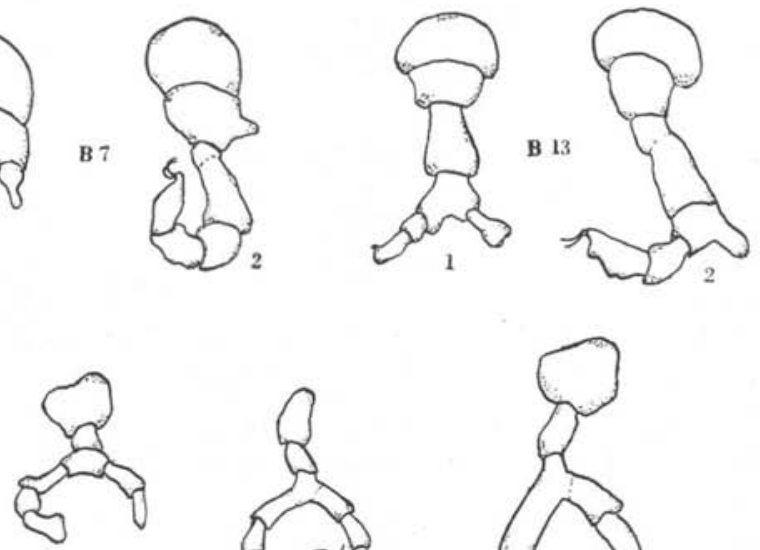

1

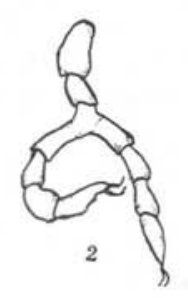

B 15

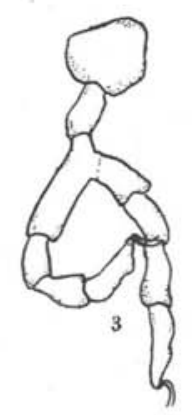

Fıg. 113. - Persistance de la schizomélie chez O. parkeri. B 7 : 1 , après une mue ; 2 , après deux mues ; B $13: 1$, après une mue ; 2 , après deux mues; $B 15: 1$, après une mue; 2 , après deux mues ; 3 , après trois mues. 
à trois reprises, mais il n'y a plus eu de mue malgré l'absence d'orifice génital. Nous pensons que l'animal était arrivé au stade adulte et que la brûlure qui avait atteint la région génitale avait empêché la formation de la vulve (fig. 108).

B 7. - La femelle issue de la deuxième mue a le même aspect que la nymphe : le trochanter porte un petit article surnuméraire qui n’a pas évolué (fig. 113).

B 13. - Chez la femelle, la branche principale de la patte a repris un aspect normal, la branche secondaire est restée sous forme d'un moignon garni d'une couronne de poils (fig. 113).

B 15. - A la deuxième mue, après la brûlure, la troisième patte droite est toujours bifurquée, et les deux branches, à peu près égales, portent des griffes; la quatrième patte est toujours absente. La nymphe est nourrie à nouveau et donne une femelle possédant les mêmes caractères. Ici encore, la régulation a joué dans le sens de la normalisation des deux branches (fig. 113).

B 19. - Après la deuxième mue, la deuxième et la troisième pattes droites manquent chez le mâle.

B 20. - Le résultat est identique au précédent, les deux pattes manquent et sont remplacées par deux cavités dans la cuticule.

B 22. - Ici encore, après la seconde mue, la troisième et la quatrième pattes droites manquent chez la femelle.

\section{Conclusions}

La schizomélie persiste jusque chez l'adulte, quel que soit le stade auquel a eu lieu la brûlure. S'il n'y a qu'une ébauche de bifurcation, l'aspect de la patte reste inchangé au stade suivant; si les deux branches sont bien développées, elles tendent de plus en plus vers l'homodynamie aux stades ultérieurs. La patte disparaît complètement si la brûlure a lésé trop profondément la région formatrice. La schizomélie se comporte donc ici comme un caractère acquis.

\section{Hérédité des schizomélies.}

Nous avons croisé la femelle B 4 (bifurcation de la première patte gauche) avec le mâle B 5 (bifurcation de la première patte droite). La ponte a eu lieu normalement, ainsi que l'éclosion. Nous avons étudié la morphologie des larves, puis des nymphes, et n'avons observé aucune anomalie.

Nous avons également obtenu des descendants de la femelle B 8 (bifurcation de la deuxième patte droite) et du mâle B 14 (bifurca- 
tion de la quatrième patte gauche), et tous étaient normaux. Nous pouvons done en conclure que la schizomélie des appendices n'est pas un caractère héréditaire, ce qui aurait d'ailleurs été fort surprenant.

\section{Discussion}

Nous produisons avec régularité des schizomélies par brûlure chez Ornithodorus parkeri ; nous en obtenons également chez d'autres espèces, qui sont parfois moins favorables, mais néanmoins nous n'avons jamais encore rencontré d'échec total. Ces schizomélies apparaissent identiques à celles qui ont été signalées dans la nature et obéissent comme elles aux lois de Bateson. Elles sont un véritable caractère acquis permanent, mais non héréditaire. La patte brûlée n'est pas toujours celle qui se dédouble, c'est quelquefois la patte voisine, précédant ou suivant l'endroit atteint.

Nous pensons donc qu'il peut $\mathbf{y}$ avoir induction à distance des schizomélies, comme dans le cas des transplantations d'appendices chez Tenebrio molitor.

Ceci nous parait confirmé par le fait que nous avons obtenu une bifurcation du trochanter après brûlure du tarse, ce qui n'aurait pas été possible si seule l'atteinte directe était en jeu.

\section{Expériences sur les Ixodidæ}

\section{$1^{\circ}$ Nymphes de Hyalomma excavatum :}

Nous avons brûlé la hanche de 12 nymphes gorgées depuis 20 jours, toutes sont mortes avant la mue.

$2^{\circ}$ Nymphes de Dermacentor pictus :

a) Sur 12 exemplaires gorgés le jour même, quatre meurent avant de muer, les autres ne régénèrent aucunement les pattes brûlées.

b) Sur 12 autres exemplaires gorgés depuis deux jours, quatre meurent, sept ne régénèrent pas, un possède sur la première hanche gauche une protubérance chitinisée (fig. 114). Après éclaircissement de la préparation, nous avons constaté que la patte était en réalité entière, mais comprimée de telle sorte qu'on ne pouvait reconnaitre aucun article, sauf l'extrémité du tarse avec pulville et griffes. Cette malformation rappelle une anomalie naturelle que nous avons décrite plus haut [ef. Amblyomma variegatum (fig. 98)]. La $2^{\circ}$ et la $3^{\circ}$ pattes manquent.

c) Sur huit exemplaires, un a une patte manquante, sept ont des pattes auxquelles il manque un ou deux articles. 


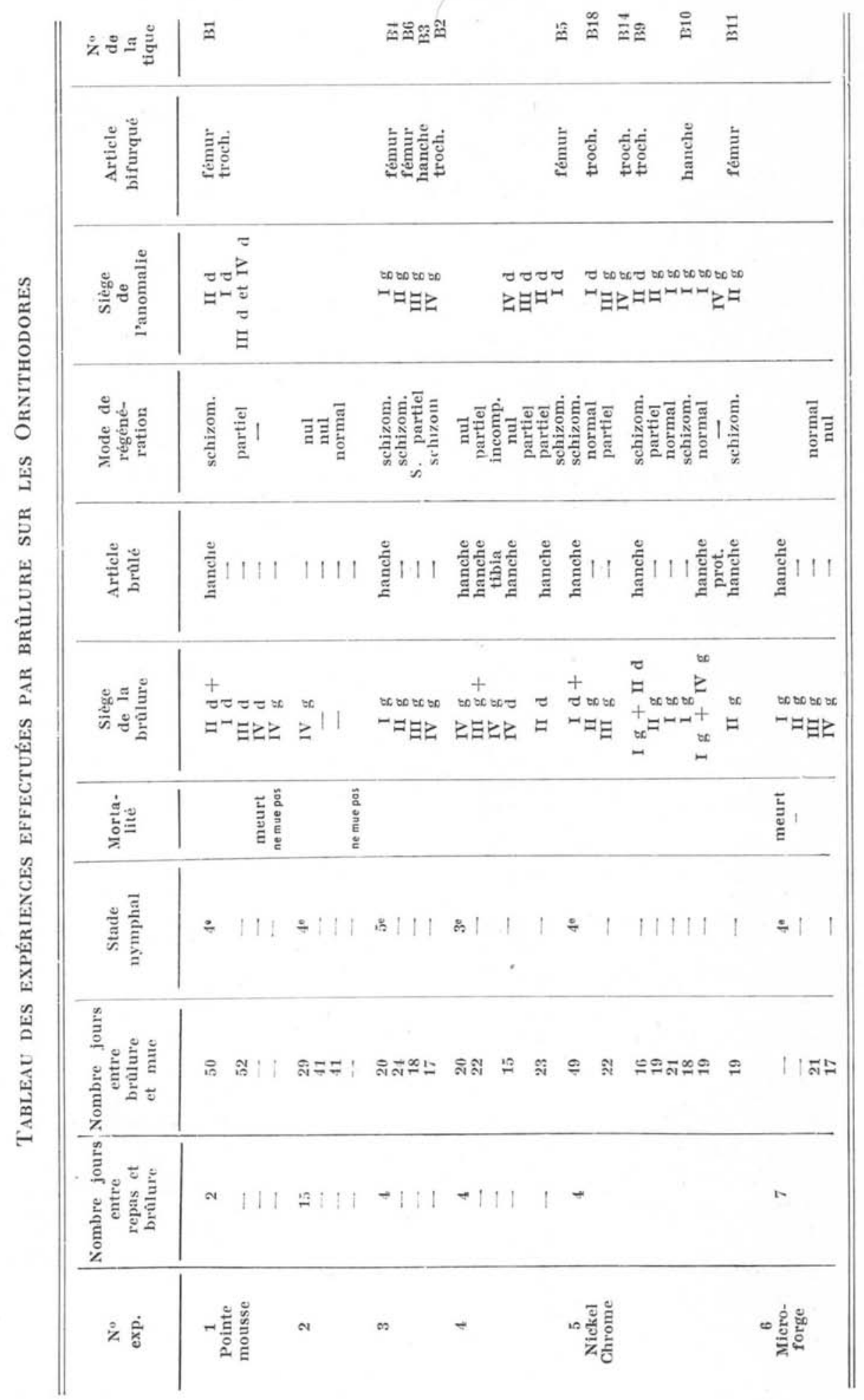




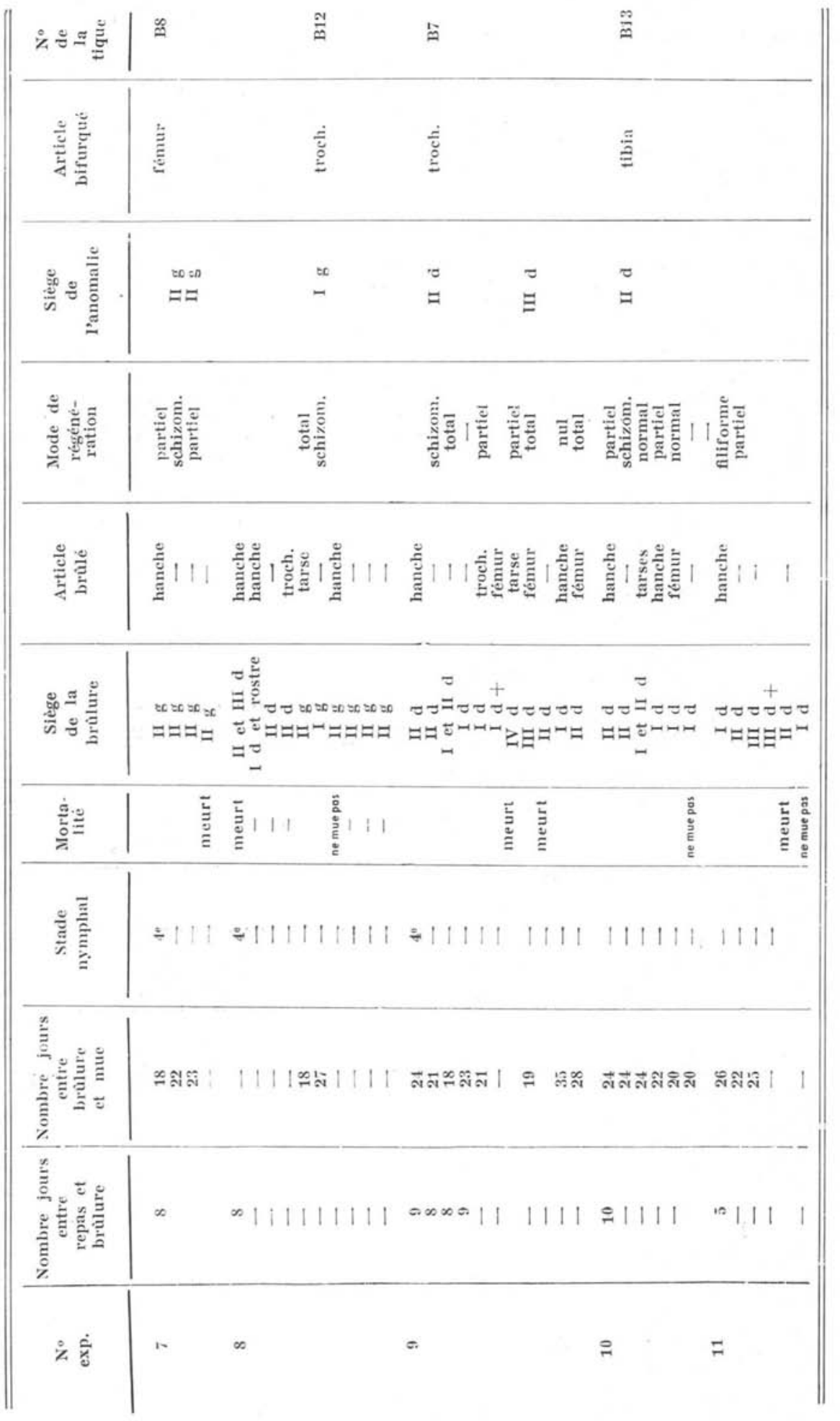




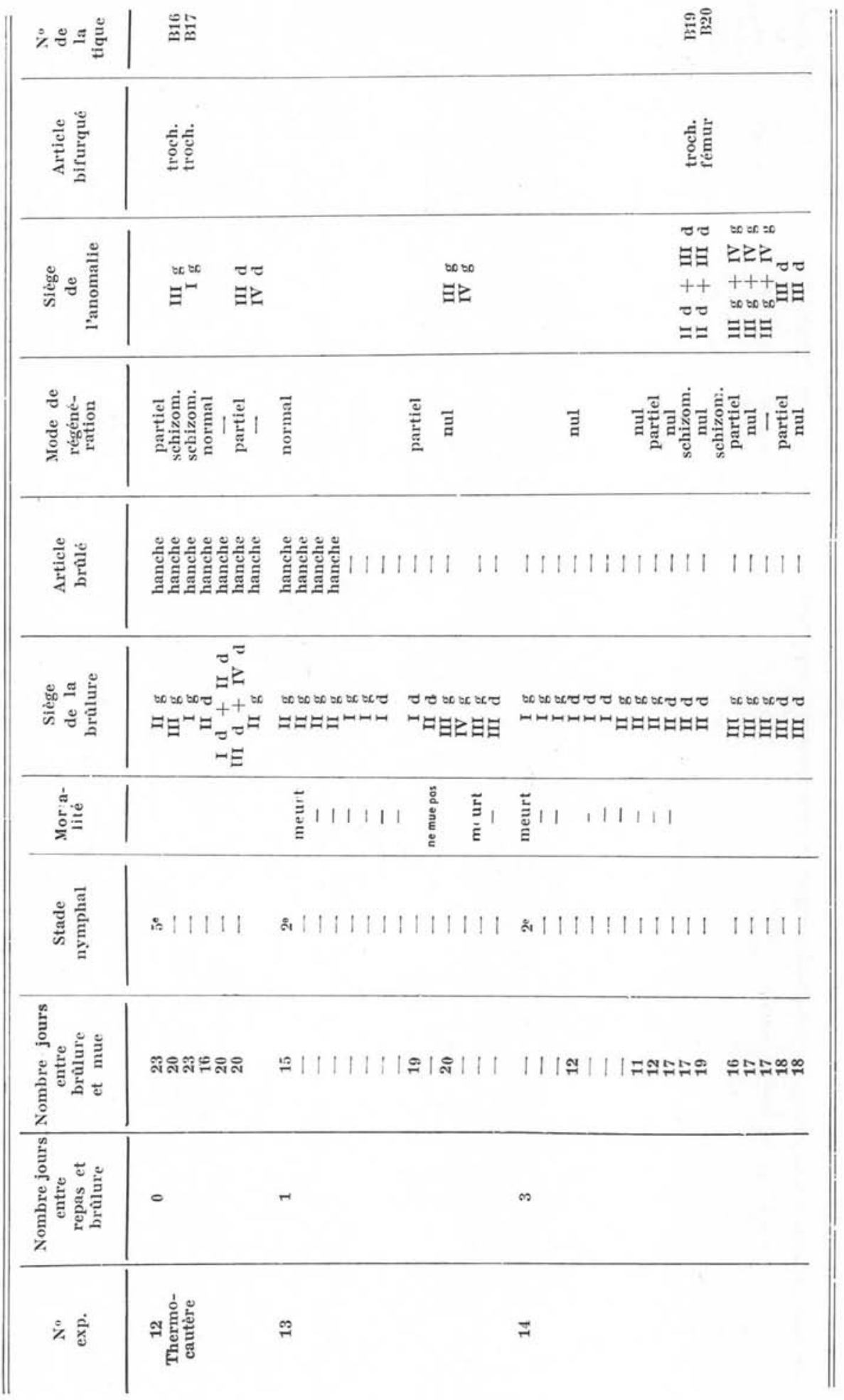




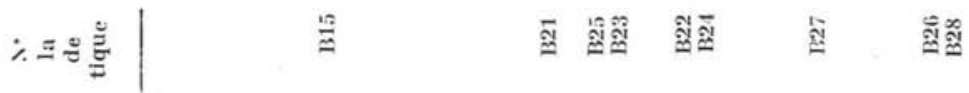

䜦苞

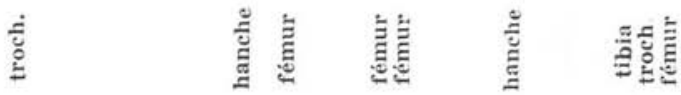

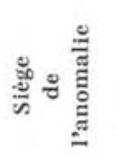

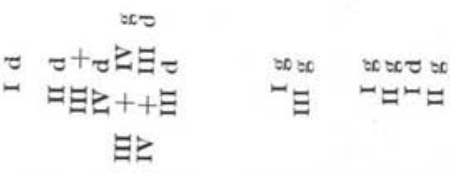

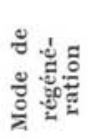

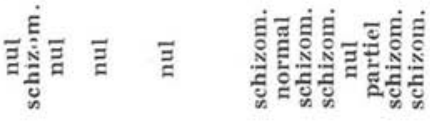

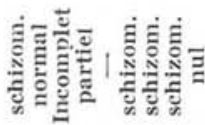

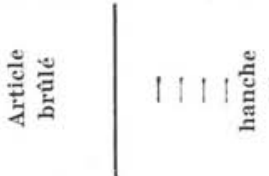

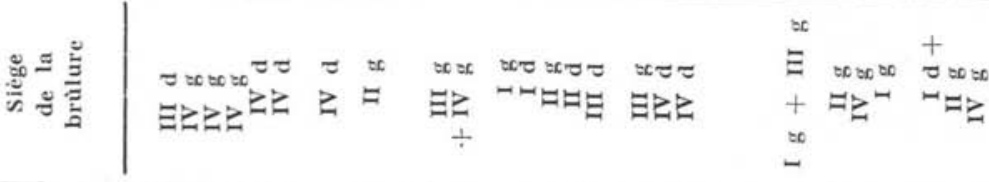

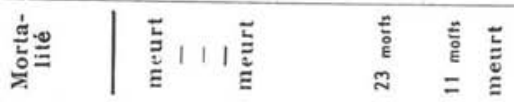

芯言

a $11111+1+1=1111111 \div \div 1$

音

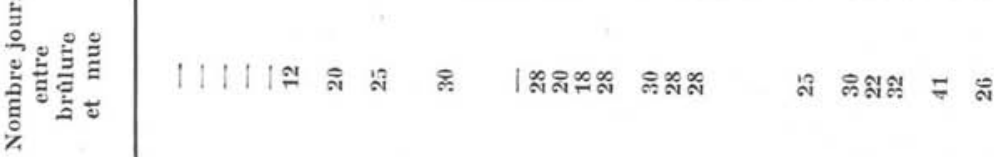

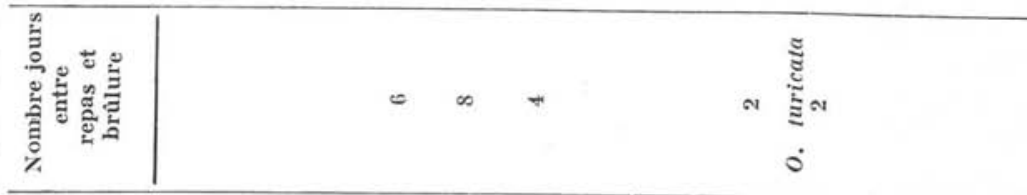

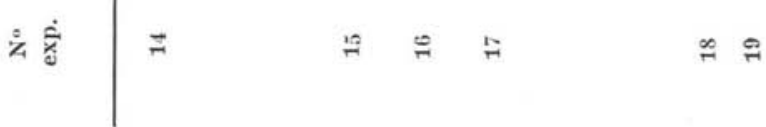




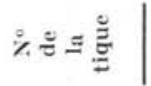

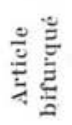

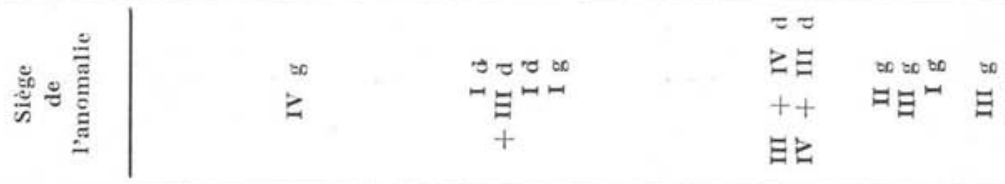

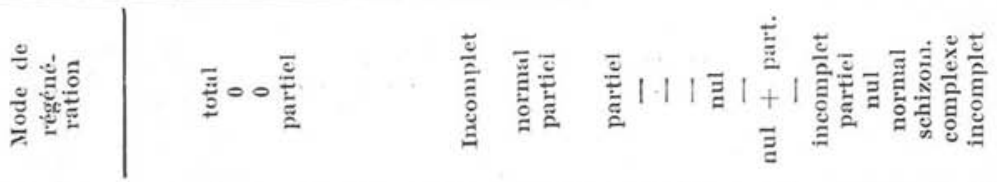

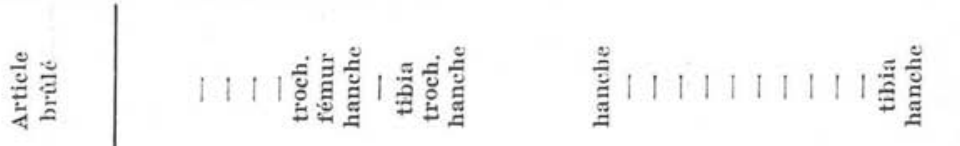

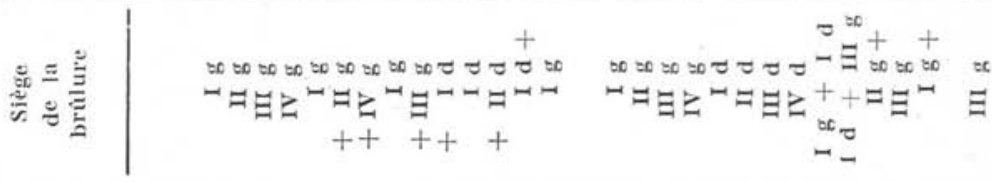

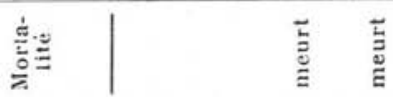

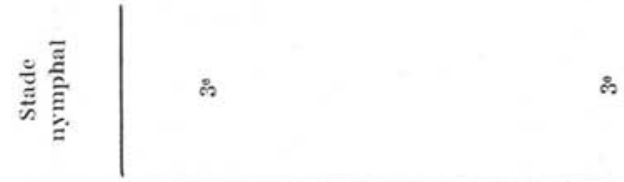

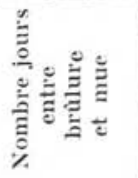

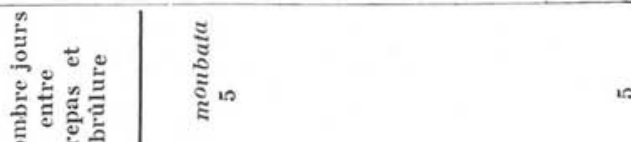

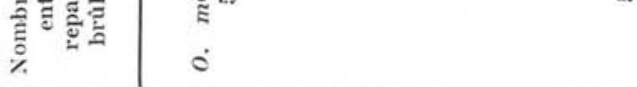

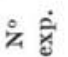

สิ

สี 
$3^{\circ}$ Nymphes de Rhipicephalus sanguineus :

Sur 24 exemplaires, 12 sont morts, un a toutes ses pattes, 11 ne présentent aucune régénération des pattes brûlées.

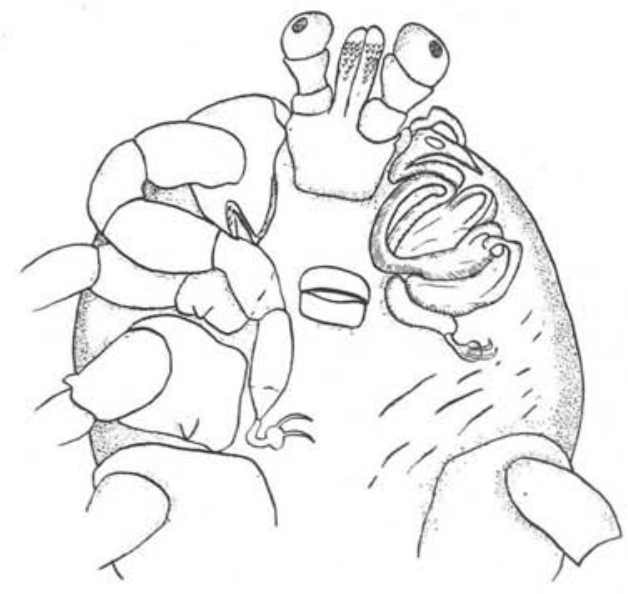

FIG. 114. - Dermacentor pictus. Atrophie de la $1^{\text {re }}$ patte gauche et ectromélie des deux suivantes après brùlure au stade nymphal.

\section{$4^{\circ}$ Larves de Dermacentor andersoni.}

Un premier lot de 12 larves gorgées le jour même meurt avant de muer. Pour le deuxième lot, gorgé la veille, trois larves sur douze résistent; deux auxquelles nous avions brûlé la troisième patte droite n'ont ni $3^{\circ}$, ni $4^{\circ}$ patte ; pour la dernière, qui avait eu la $2^{\circ}$ patte droite brûlée, il manque trois pattes.

Nous n'avons donc pu produire aucune schizomélie chez les Ixodidæ. Les pattes régénérées après brûlure sont complètes ou atrophiées, mais jamais divisées ; le plus souvent, d'ailleurs, la régénération ne se fait pas et la patte brûlée manque, ainsi quelquefois que la patte qui lui fait suite. Le pouvoir régénérateur de ces animaux paraît bien inférieur à celui des Ornithodores; c'est sans doute ce qui explique que tous les cas d'ectromélies signalés par divers auteurs ont toujours trait à des Ixodidx. Cette anomalie a été attribuée parfois à un accident embryonnaire, les traumatismes ultérieurs devant permettre une régénération au moins partielle des appendices lésés.

Or, dans toutes les expériences ci-dessus, nous avons obtenu des exemplaires dont la patte brûlée avait complètement disparu, sans 
même laisser l'emplacement de la hanche chitineuse ; la cuticule était semblable à celle du reste de l'abdomen. Nous pouvons donc admettre que, sauf dans les cas où une patte manquérait dès le stade larvaire (ce qui n'a jamais été signalé à notre connaissance), toute disparition d'appendice est due à un facteur externe, traumatique, ayant lieu à un moment quelconque de la vie larvaire ou nymphale.

\section{Action des rayons ultra-violets}

Avec les ultra-violets, on peut espérer obtenir une action sur l'œuf sans empêcher son évolution.

Geigy (1926-27-31) a utilisé cette méthode sur des œufs de Drosophile. En irradiant la région dorsale, il obtient surtout des anomalies de la segmentation ; l'irradiation de la région ventrale produit au contraire des anomalies des appendices n'apparaissant que chez l'adulte: certaines pattes sont absentes ou rudimentaires, d'autres peuvent présenter des schizomélies binaires ou ternaires. Des essais sur les larves n'ont pas donné de formations analogues.

Geigy et Lüscher (1942) ont irradié des œufs de Tineola biselliella et ont également obtenu quelques duplications de pattes chez l'adulte.

Nous avons soumis à l'irradiation des femelles gorgées d'Ornithodorus parkeri; elles ont pondu ensuite normalement et nous n'avons observé aucune anomalie dans la descendance.

Nous avons ensuite irradié des œufs de Rhipicephalus sanguineus. Ces œufs ont éclos normalement, mais les larves ne se sont pas gorgées.

Dans notre dernière série d'expériences, nous avons irradié des œufs d'Ornithodorus parkeri. Les larves ont éclos normalement et ne présentent pas de malformations. Nous n'avons pu les amener au stade adulte.

Pour ces diverses opérations, nous nous sommes servie d'une lampe très faible (2 watts). Il faudrait utiliser des radiations plus fortes et en pinceau, nécessitant un appareillage spécial.

\section{Action des rayons $X$}

Nous abordons ici un mode d'expérimentation qui s'écarte considérablement des processus tératogénétiques possibles dans les conditions naturelles. Les généticiens l'ont souvent utilisé avec succès pour obtenir des mutations. 
Nous avons tenté diverses expériences sur des femelles gorgées de Rhipicephalus sanguineus et d'Ornithodorus parkeri et sur des œufs des mêmes espèces. Le développement a toujours été normal et les Tiques n'ont absolument pas réagi au traitement. Nous en avons conclu que l'appareil employé émettait des rayons trop pénétrants qui traversaient l'œuf ou même la femelle sans rien léser.

Nous avons pu utiliser ultérieurement un appareil extrêmement précis de l'Institut du Radium, pavillon Pasteur, grâce à l'obligeance de MM. Lacassagne et Lartarjet à qui nous exprimons ici nos vifs remerciements, ainsi qu'à $M$. Morenne dont l'extrême complaisance nous a permis de mener à bien ces expériences.

Nous avons soumis nos Tiques au rayonnement d'un tube Beaudouin-Holweck à anticathode de molybdène, $33 \mathrm{KV}$, rayonnement filtré par une fenêtre de $0,05 \mathrm{~mm}$. d'aluminium. Longueur d'onde moyenne $0,9 \dot{A}$. Intensité au tube 15 milliampères. Nous avons fait varier les doses de rayonnement pour chaque expérience, toutes les autres conditions restant les mêmes. Les Tiques expérimentées appartiennent toutes à l'espèce Ornithodorus parkeri.

\section{Expériences sur les œufs :}

Il s'agissait d'irradier des œufs à divers stades de leur développement, avec l'espoir que telle ou telle partie de l'embryon serait détruite et provoquerait l'apparition de larves atrophiées ou monstrueuses. Il nous a été impossible d'orienter ces œufs qui sont subsphériques et assez opaques. Les premières expériences ont surtout eu pour but de rechercher les doses utiles, étant donné que nous n'avions aucune base de départ. Nous cherchions une dose provoquant une mortalité d'environ $50 \%$.

Expérience $n^{\circ} 1$ :

Nous avons pris plusieurs lots d'une quarantaine d'œufs provenant d'une même ponte et les avons soumis à des irradiations de $1.000,2.000,5.000,10.000,15.000$ et $25.000 \mathrm{r}$. Aucun n'a éclos. Mais parmi les-témoins, la mortalité avait été extrêmement forte, puisque sur 52 œufs, 10 seulement avaient éclos et deux larves avaient survécu. Le seul résultat pratique de cette première expérience a été de montrer que des œufs trop jeunes ne résistent pas aux manipulations pourtant réduites qui consistent à sortir une ponte de son tube et à la fragmenter en plusieurs lots. Ajoutons que les quelques œufs qui étaient restés dans le tube initial avec les parents se sont parfaitement développés. Seule, la manipulation était donc en cause. 
Les œufs provenaient d'une femelle qui avait commencé à pondre quatre jours auparavant ; la ponte durant plusieurs jours, nous avions donc des œufs âgés de un à quatre jours.

Expérience $n^{\circ} 2$ :

Avec des œufs âgés de 3 à 7 jours, nous avons donné des doses fortes :

$\begin{array}{cc}\text { Irradiation } & \text { Eclosion } \\ - & - \\ 15.000 \mathrm{r} & 2 \% \\ 25.000 \mathrm{r} & 0 \% \\ 40.000 \mathrm{r} & 1 \% \\ 50.000 \mathrm{r} & 0 \% \\ 72.000 \mathrm{r} & 0 \%\end{array}$

Ces lots comportaient 70 à 90 œufs. Dans le lot 1 , nous avons récolté deux larves qui n'ont pas vécu et, dans le lot 3 , une seule larve également morte. Ici encore, chez les témoins, 15 larves seulement ont éclos.

Expérience $n^{\circ} 3$ :

Avec des œufs un peu plus âgés, 9 à 13 jours, nous rééditons l'expérience précédente :

\begin{tabular}{|c|c|c|}
\hline & Irradiation & Eclosion \\
\hline ot $1 \ldots \ldots \ldots \ldots$ & $20.000 \mathrm{r}$ & $6 \%$ \\
\hline $2 \ldots \ldots \ldots \ldots$ & $25.000 \mathrm{r}$ & $3 \%$ \\
\hline $3 \ldots \ldots \ldots \ldots$ & $30.000 \mathrm{r}$ & $4 \%$ \\
\hline $4 \ldots \ldots \ldots \ldots$ & $35.000 \mathrm{r}$ & $2 \%$ \\
\hline
\end{tabular}

Dans chaque lot, il éclôt quelques larves qui meurent rapidement; il y a à peu près $50 \%$ d'éclosions chez les témoins.

Expérience $n^{\circ} 4$ :

Nous employons des œufs de 8 à 11 jours avec des doses plus faibles :

\section{Irradiation}

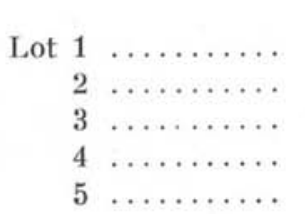

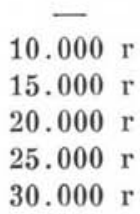

Eclosion

$\begin{aligned} 93 & \% \\ 57 & \% \\ 23 & \% \\ 20 & \% \\ 3 & \%\end{aligned}$


Les témoins ont éclọs à $75 \%$.

Nous avons eu ici des éclosions dans tous les lots, avec un pourcentage régulièrement décroissant de 1 à 5 . Nous reviendrons sur les résultats ultérieurement.

Nous avons considéré que les doses de 10.000 à $15.000 \mathrm{r}$. devaient être les plus favorables et nous avons essayé des doses plus rapprochées.

Expérience $n^{\circ} 5$ :

Cufs de 8 à 12 jours :

\begin{tabular}{|c|c|c|c|}
\hline & & Irradiation & $\begin{array}{c}\text { Eclosion } \\
-\end{array}$ \\
\hline Lot 1 & $\ldots \ldots \ldots \ldots$ & $8.000 \mathrm{r}$ & $70 \%$ \\
\hline 2 & $\ldots \ldots \ldots \ldots$ & $9.000 \mathrm{r}$ & $50 \%$ \\
\hline 3 & $\ldots \ldots \ldots \ldots$ & $10.000 \mathrm{r}$ & $60 \%$ \\
\hline 4 & $\ldots \ldots \ldots \ldots$ & $11.000 \mathrm{r}$ & $5 \%$ \\
\hline 5 & $\ldots \ldots \ldots \ldots$ & $12.000 \mathrm{r}$ & $4 \%$ \\
\hline
\end{tabular}

Le pourcentage d'éclosion est sensiblement le même pour les trois premiers lots. Les résultats très différents obtenus chez les deux derniers lots ne proviennent certainement pas de la dose d'irradiation un peu plus forte qu'ils ont reçue, mais ces deux lots provenaient d'une ponte différente, plus âgée de deux jours.

Nous n'avons pas poursuivi plus loin ces expériences sur les œufs, les résultats nous paraissant vraiment trop irréguliers. En effet :

$1^{\circ}$ Le pourcentage d'éclosion est très variable, aussi bien chez les témoins que chez les lots irradiés.

$2^{\circ}$ L'âge exact de l'œuf est difficilement déterminable, la ponte durant plusieurs jours. Il faudrait done isoler chaque jour les œufs pondus; or, nous avons vu que cette simple manipulation risque de les faire sécher.

$3^{\circ}$ Une même dose arrive à donner des résultats très différents suivant les lots; dans ces conditions, une expérimentation précise devient impossible.

Etudions maintenant les résultats obtenus avec les survivants des deux dernières expériences:

Expérience $n^{\circ} 4$ :

Nous avons nourri parallèlement des témoins et les divers lots.

Pour le lot 1, les survivants ont tous accepté le repas, mais cinq seulement ont mué et ont alors continué leur évolution normale- 
ment. Nous remarquons que, sur cinq spécimens, quatre ont donné des adultes après la $3^{\mathrm{e}}$ mue nymphale, alors que chez les témoins il y avait à la même période seulement $64 \%$ d'adultes. Il est possible que l'irradiation ait activé le développement, mais, sur un aussi petit nombre d'individus, on doit se garder de conclure.

Pour le lot 2, 25 larves se sont gorgées, mais une seule a mué et a ensuite continué son cycle.

Pour les lots 3, 4 et 5, dix-huit, trois et un individus se sont gorgés et aucune mue n'a suivi le repas.

Tous les exemplaires n'ayant pas mué sont morts assez rapidement.

Nous voyons donc que l'action léthale des rayons $\mathrm{X}$ se prolonge durant un certain laps de temps, puisque des larves qui paraissaient en très bon état au moment du repas ne peuvent arriver à muer.

Le phénomène est encore plus remarquable pour l'expérience $n^{\circ} 5$. Dans le lot 1 , une seule larve s'est gorgée et est morte ensuite. Dans le lot 3, sur 80 larves gorgées, quatre seulement ont mué. Dans les autres lots, les larves ne se sont pas gorgées.

Etude de la descendance du lot 1 (expérience $\mathrm{n}^{\circ} 4$ ) :

Nous avons obtenu des pontes des adultes ( 3 \& et $\left.1 \delta^{7}\right)$. Ces pontes ont éclos normalement ; nous n'avons rien observé de particulier sur le stade larvaire, ni sur les deux premiers stades nymphaux. Nous ne constatons ni mortalité anormale, ni particularités morphologiques donnant l'indice d'une mutation. Rien non plus chez les adultes.

\section{Expériences sur les femelles:}

Nous pensions, en agissant sur des femelles gorgées, intervenir sur l'œuf dès le début de son développement et éviter la mortalité élevée due aux manipulations.

Nous avons employé des doses élevées de 20 à $30.000 \mathrm{r}$. et toutes nos femelles sont mortes. Nous n'avons malheureusement pas pu recommencer ces expériences.

\section{Expériences sur les larves :}

En expérimentant sur des larves gorgées, nous pensions obtenir des résultats beaucoup plus réguliers qu'avec les œufs; les larves sont en effet résistantes et la mortalité est très faible dans un élevage normal. En outre, on peut faire varier non seulement le degré d'irradiation, mais encore le moment de cette irradiation par rapport au repas et par conséquent à la mue qui survient au bout de 9 à 10 jours. 
Expérience $n^{\circ} 1$ :

Larves gorgées depuis cinq jours. Nous prenons des lots d'une quarantaine d'individus.

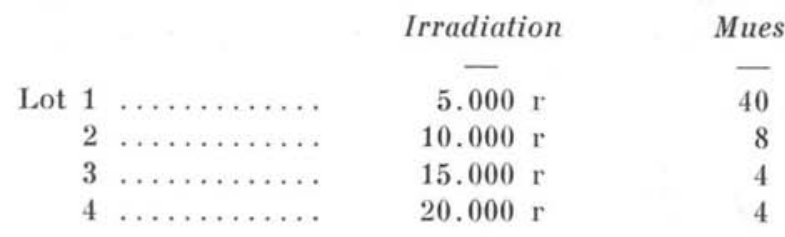

Dans le lot 1, les larves muent en même temps que les témoins ; dans le lot 2 , il y a seulement quelques mues; quatre mues dans le lot 3 et quatre mues tardives dans le lot 4 donnent des nymphes très petites et qui meurent rapidement.

12 nymphes du lot 1 se nourrissent à nouveau, mais meurent sans avoir mué.

Expérience $n^{\circ} 2$ :

Larves gorgées depuis trois jours.

\begin{tabular}{|c|c|c|c|}
\hline & & Irradiation & Mues \\
\hline Lot 1 & $\ldots \ldots \ldots \ldots \ldots$ & $10.000 \mathrm{r}$ & Pas de mues \\
\hline 2 & $\ldots \ldots \ldots \ldots \ldots$ & $15.000 \mathrm{r}$ & $\gg$ \\
\hline 3 & $\ldots \ldots \ldots \ldots \ldots$ & $20.000 \mathrm{r}$ & $\gg$ \\
\hline
\end{tabular}

Expérience $n^{\circ} 3$ :

Larves gorgées depuis cinq jours.

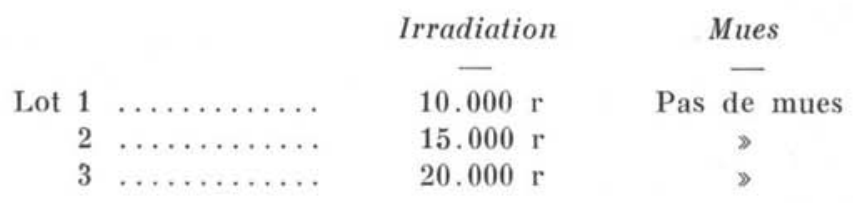

Dans ces deux expériences, les doses étaient trop fortes et toutes les larves sont mortes avant de muer.

Expérience $n^{\circ} 4$ :

Nous prenons ici une même dose, soit 4.000 r. (cette dose correspond à ce qu'on emploie généralement pour les Drosophiles), et nous opérons sur des larves gorgées depuis des temps différents. 


\begin{tabular}{|c|c|c|c|c|c|c|}
\hline \multirow[b]{2}{*}{ bot } & & Temps écoulé & & lues & $\begin{array}{c}\text { Repas } \\
\text { nymphal }\end{array}$ & $2^{\mathrm{e}}$ mue \\
\hline & $\ldots \ldots$ & 1 jour & Pas & de mue & & \\
\hline 2 & $\ldots \ldots$ & $2 \gg$ & & $\gg$ & & \\
\hline 3 & $\ldots \ldots$ & $3 \gg$ & & $\gg$ & & \\
\hline 4 & $\ldots \ldots$ & $4 \gg$ & Mue & normale & + & 一 \\
\hline 5 & $\ldots \ldots$ & $6 \gg$ & & $\gg$ & + & 一 \\
\hline 6 & $\ldots \ldots$ & $7 \gg$ & & $\gg$ & + & 一 \\
\hline 7 & $\ldots \ldots$ & $8 \gg$ & & $\gg$ & + & 一 \\
\hline
\end{tabular}

Les lots 1, 2 et 3 ne muent pas et meurent assez rapidement. Les quatre autres muent normalement en même temps que les témoins. La première hypothèse que suggère ce résultat est que les larves récemment gorgées sont plus sensibles que les autres. Mats, quand nous nourrissons les nymphes issues de la première mue, nous constatons que celles-ci ne peuvent pas muer une seconde fois et meurent à leur tour. Nous constatons donc ici encore l'action progressive de l'irradiation.

Expérience $n^{\circ} 5$ :

Nous diminuons encore la dose d'irradiation et opérons sur des larves nourries depuis cinq jours.

\begin{tabular}{|c|c|c|c|c|c|c|}
\hline & & Irrodiations & $\begin{array}{c}\text { Morts oprès le } \\
1^{\text {or repas }} \\
\text { nymphal }\end{array}$ & Vivants & $\begin{array}{l}2^{2} \mathrm{repos} \\
\text { nymphol }\end{array}$ & $\begin{array}{c}\text { Vivonts après } \\
\text { I'irradiation } \\
\text { (3 mois) }\end{array}$ \\
\hline Lot 1 & $\ldots \ldots$ & $1.000 \mathrm{r}$ & 0 & 27 & 5 & 26 \\
\hline 2 & $\ldots \ldots$ & $2.000 \mathrm{r}$ & 24 & 7 & 0 & 6 \\
\hline 3 & $\ldots \ldots$ & $2.000 \mathrm{r}$ & 5 & 18 & 1 & 17 \\
\hline 4 & $\ldots \ldots$ & $3.000 \mathrm{r}$ & 27 & 22 & 0 & 0 \\
\hline 5 & $\ldots \ldots$ & $4.000 \mathrm{r}$ & 20 & 9 & 0 & 0 \\
\hline
\end{tabular}

Tous les lots comprenant une trentaine d'individus ont mué normalement et donné des nymphes qui se sont gorgées. Entre ce repas et la mue, la mortalité a été de 0 pour le lot 1,24 pour le lot 2 , 5 pour le lot 3,27 pour le lot 4 et 20 pour le lot 5 .

Au $2^{\circ}$ repas, cinq individus du lot 1 se nourrissent et un du lot 3 ; les autres refusent le repas. Trois mois après l'irradiation, il y a 26 survivants dans le lot 1, 6 dans le lot 2,17 dans le lot 3 et aucun dans les lots 4 et 5 . Mais tous ces survivants, sauf six, en sont restés au premier stade nymphal, n'ont pas mué depuis le premier repas nymphal et ne se sont pas nourris. 
Les conclusions que nous pouvons tirer de ces expériences sont les suivantes:

$1^{\circ}$ Les Tiques sont extrêmement sensibles à l'action des rayons $\mathrm{X}$ : des doses bien inférieures à celles que l'on utilise pour les Drosophiles provoquent une mortalité extrêmement élevée.

$2^{\circ}$ Les rares survivants de ces expériences paraissent se comporter normalement ; ni eux, ni leur descendance ne présentent d'anomalies. L'action des rayons $\mathrm{X}$ paraît donc léthale, mais non tératogénique. Les mutations devraient apparaitre à la génération suivante ; or, nous n'avons rien constaté.

TABLEAU DES EXPÉRIENCES D'irRadiation SUR LES EUfS

\begin{tabular}{|c|c|c|c|c|c|c|}
\hline $\begin{array}{c}\text { NUMÉRo } \\
\text { DE } \\
\text { L'EXPÉR. }\end{array}$ & Dose EN R & $\begin{array}{c}\text { AGE } \\
\text { DES EUFS }\end{array}$ & Éclosion & $\begin{array}{l}\text { NoMbre } \\
\text { D'INDIVIDUS } \\
\text { REPAS } \\
\text { LARVAIRE }\end{array}$ & & PremtÈre MUE \\
\hline 1 & $\begin{array}{c}1.000 \\
2.000 \\
5.000 \\
10.000 \\
15.000 \\
25.000 \\
\text { Témoins }\end{array}$ & $1-4 \mathrm{j}$ & $\begin{array}{l}0 \\
n \\
n \\
n \\
n \\
20 \\
20\end{array}$ & & & \\
\hline 2 & $\begin{array}{c}15.000 \\
25.000 \\
40.000 \\
50.000 \\
72.000 \\
\text { Témoins }\end{array}$ & $3-7 \mathrm{j}$ & $\begin{array}{l}2 \% \\
0 \\
1 \% \\
0 \\
0 \\
15 \%\end{array}$ & $\begin{array}{l}0 \\
0\end{array}$ & & \\
\hline 3 & $\begin{array}{c}20.000 \\
25.000 \\
30.000 \\
35.000 \\
\text { Témoins }\end{array}$ & $9-13 \mathrm{j}$ & $\begin{aligned} 6 & \% \\
3 & \% \\
4 & \% \\
2 & \% \\
50 & \%\end{aligned}$ & $\begin{array}{l}0 \\
0 \\
0 \\
0 \\
0\end{array}$ & & \\
\hline 4 & $\begin{array}{c}10.000 \\
\\
15.000 \\
20.000 \\
25.000 \\
30.000 \\
\text { Témoins }\end{array}$ & $8-11 j$ & $\begin{aligned} 93 \% \\
\\
57 \% \\
23 \% \\
20 \% \\
3 \% \\
75 \%\end{aligned}$ & \begin{tabular}{r|r}
28 & \\
& \\
25 & \\
18 & \\
3 & \\
1 &
\end{tabular} & $\begin{array}{l}\mathbf{5} \\
\mathbf{1} \\
\mathbf{0} \\
0 \\
\mathbf{0}\end{array}$ & $\begin{array}{l}\longrightarrow \text { adult. }- \\
\stackrel{2}{\longrightarrow} \text { Gén. norm. } \\
\longrightarrow \quad \text { adultes. }\end{array}$ \\
\hline 5 & $\begin{array}{c}8.000 \\
9.000 \\
10.000 \\
11.001 \\
12.000 \\
\text { Témoins }\end{array}$ & $8-12 \mathrm{j}$ & $\begin{aligned} 70 \% \\
50 \% \\
60 \% \\
5 \% \\
4 \%\end{aligned}$ & $\begin{array}{r}1 \\
0 \\
80 \\
0 \\
0\end{array}$ & 0 & \\
\hline
\end{tabular}


$3^{\circ}$ L'action léthale n'est pas immédiate :

a) l'irradiation précoce d'un individu à un stade donné arrête son évolution avant la fin de ce stade; un œuf irradié au début de son évolution n'éclôt pas; une larve irradiée dans les trois jours suivant son repas ne mue pas ;

b) l'irradiation tardive d'un individu à un stade donné n'arrête son évolution qu'au stade suivant; un œuf irradié tardivement donnera une larve qui se gorgera, mais ne pourra muer ; une larve irradiée tardivement donnera une nymphe au premier stade, qui se gorgera, mais ne pourra muer.

$4^{\circ}$ L'action léthale est limitée dans le temps. Après une irradiation aussi bien précoce que tardive, les individus qui survivent à la période critique continuent leur évolution sans subir de nouvelle mortalité, arrivent au stade adulte et se reproduisent normalement.

TABLEAU DES EXPÉRIENCES D'IrRAdiation SUR LES LARVES GORGÉES

\begin{tabular}{|c|c|c|c|c|c|c|c|c|}
\hline $\begin{array}{c}N \cdot \\
\text { DE } \\
\text { L'EXP. }\end{array}$ & $\begin{array}{l}\text { Dose } \\
\text { EN } \mathrm{R}\end{array}$ & $\begin{array}{c}\text { NoMBRE } \\
\text { DE JOUHS } \\
\text { ENTRE } \\
\text { REPAS ET } \\
\text { EXPÉR. }\end{array}$ & 1" MUE & $\begin{array}{c}1 \text { 1" REPAS } \\
\text { NYMPHAL. } \\
\text { NOMBRE } \\
\text { D'INDIVI- } \\
\text { DUS }\end{array}$ & MORTs & $2^{*} \mathrm{MUE}$ & $\begin{array}{l}2^{*} \mathrm{R} . \\
\mathrm{NY} \text {. }\end{array}$ & $\begin{array}{c}\text { S URVIE } \\
3 \text { MOIS } \\
\text { NB. } \\
\text { D'IN- } \\
\text { DIV. }\end{array}$ \\
\hline 1 & $\begin{array}{r}5.000 \\
10.000 \\
15.000 \\
20.000\end{array}$ & 5 & $\begin{array}{r}100 \% \\
20 \% \\
10 \% \\
10 \%\end{array}$ & $\begin{array}{r}12 \\
0 \\
0 \\
0\end{array}$ & 12 & & & \\
\hline 2 & $\begin{array}{l}10.000 \\
15.000 \\
20.000\end{array}$ & 3 & $\begin{array}{l}0 \\
0 \\
0\end{array}$ & & & & & \\
\hline 3 & $\begin{array}{l}10.000 \\
15.000 \\
20.000\end{array}$ & 5 & $\begin{array}{l}0 \\
0 \\
0\end{array}$ & & & & & \\
\hline 4 & $\begin{array}{c}4.000 \\
n \\
n \\
" \\
b \\
"\end{array}$ & $\begin{array}{l}1 \\
2 \\
3 \\
4 \\
6 \\
7 \\
7 \\
8\end{array}$ & $\begin{array}{c}0 \\
0 \\
0 \\
100 \% \\
100 \% \\
100 \% \\
100 \%\end{array}$ & $\begin{array}{l}30 \\
32 \\
29 \\
30\end{array}$ & $\begin{array}{l}30 \\
32 \\
29 \\
30\end{array}$ & $\begin{array}{l}0 \\
0 \\
0 \\
0\end{array}$ & & \\
\hline 5 & $\begin{array}{l}1.000 \\
2.000 \\
2.000 \\
3.000 \\
4.000\end{array}$ & 5 & $\begin{array}{l}100 \% \\
100 \% \\
100 \% \\
100 \% \\
100 \%\end{array}$ & $\begin{array}{l}27 \\
20 \\
18 \\
22 \\
25\end{array}$ & $\begin{array}{r}1 \\
14 \\
1 \\
22 \\
25\end{array}$ & $\begin{array}{l}5 \\
0 \\
1 \\
0 \\
0\end{array}$ & $\begin{array}{l}5 \\
0 \\
1 \\
0 \\
0\end{array}$ & $\begin{array}{r}26 \\
6 \\
17 \\
0 \\
0\end{array}$ \\
\hline
\end{tabular}


$5^{\circ}$ La période critique est toujours située entre le repas et la mue suivante : les animaux se gorgent, mais ne peuvent pas muer et meurent.

6" L'irradiation parait donc intervenir comme inhibitrice de la mue. Nous ne pouvons malheureusement préciser sur quelle partie du processus elle intervient, étant donné l'état actuel de nos connaissances sur ce sujet, mais il est certain qu'il se produit un arrêt dans le déroulement normal du cycle ; cet arrêt peut se faire au moment de la digestion du sang ingéré en empêchant son passage dans l'organisme.

Nous ne pensons pas qu'il y ait un changement d'ordre morphologique de l'organisme, mais plutôt d'ordre radio-biologique. La rupture de l'équilibre normal pourrait entraîner la suppression de la production hormonale qui préside, vraisemblablement, au processus de la mue.

\section{AGENTS CHIMIQUES}

\section{Action de l'Éthylquinone}

Nous avons pu expérimenter ce produit grâce à l'obligeance de J. Balazuc qui l'utilise pour des expériences sur les Coléoptères. Il correspond à la sécrétion des adultes de Tribolium confusum, isolé par Roth et Howland, sous forme d'un liquide jaune brun, volatil, odorant, cristallisable. Le produit pur que nous employons ici est en petits cristaux, jaune orangé, brunissant à l'air, fondant à $38^{\circ} \mathrm{C}$., et correspondant à la formule :

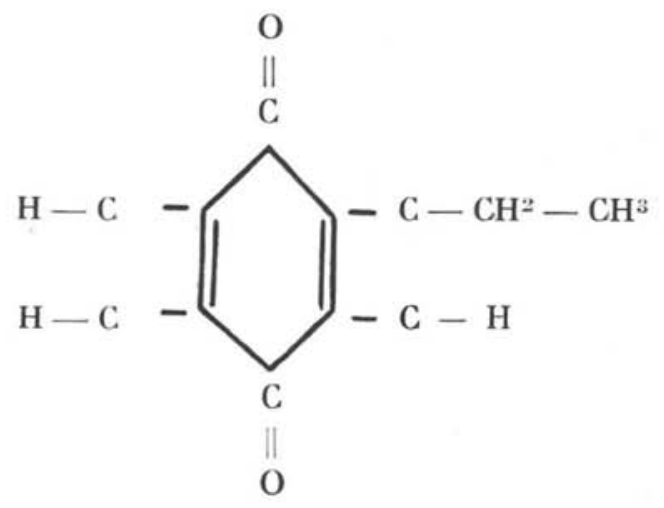

Nous avons étudié successivement l'action des cristaux, des vapeurs et d'une solution alcoolique sur différents Ixodoidea. 


\section{Expériences sur Ornithodorus parkeri}

Action des cristaux :

$1^{\circ} 20$ larves gorgées d'Ornithodorus parkeri sont mises dans un tube contenant de très petits fragments d'éthylquinone. Nous les retrouvons toutes mortes 16 heures plus tard.

$2^{\circ}$ Deux nymphes ayant eu leur troisième repas nymphal sont mises dans le même tube que ci-dessus et y séjournent trois heures. Elles sont vivantes, mais peu mobiles, et l'extrémité des pattes présente une coloration rouge. L'une mue quatre jours après l'expérience, mais ne peut sortir de l'exuvie qui paraît durcie ; l'autre mue un jour plus tard, dans les mêmes conditions ; les pattes sont peu chitinisées, mais ne présentent pas d'anomalies, non plus que les pièces buccales.

$3^{\circ} 12$ nymphes au $3^{\circ}$ stade, gorgées depuis six jours, sont mises dans un tube contenant un cristal d'éthylquinone et laissées 15 heures. Neuf meurent, une mue le $43^{\circ}$ jour, deux le $44^{\circ}$ jour.

L'une présente une ébauche de bifurcation de la première patte gauche $(Q 6)$ (fig. 115), une autre, des bifurcations de la deuxième et de la troisième pattes gauches (Q 7) (fig. 115); pour la deuxième patte, il s'agit d'une protubérance conique, située latéralement sur la partie distale du fémur, les trois derniers articles étant rejetés vers l'avant et de taille légèrement réduite; pour la troisième, de taille réduite, l'ensemble a un aspect normal, mais sur le tibia se trouve ventralement une expansion non articulée et non chitinisée.

$4^{\circ} 30$ nymphes au premier stade, gorgées depuis 24 heures, sont mises en contact 15 minutes avec un cristal pulvérisé. 27 meurent, 3 vivent et sont normales.

Ces expériences avaient surtout pour but de montrer la toxicitė de l'éthylquinone vis-à-vis des Tiques.

\section{Action des vapeurs :}

$1^{\circ}$ Six nymphes, au $2^{\circ}$ stade, gorgées depuis la veille, sont laissées une heure au contact des vapeurs. Deux muent le $19^{\circ}$ jour après l'expérience, une le $20^{\mathrm{e}}$ jour et trois le $21^{\mathrm{e}}$ jour. Toutes sont normales.

$2^{\circ}$ Six nymphes provenant du même lot sont laissées 2 heures 30 . Deux meurent, une mue le $25^{\circ}$ jour, une le $27^{\circ}$, une le $30^{\circ}$ et une le

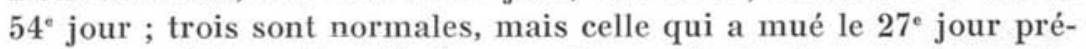
sente une bifurcation de la première patte droite au niveau du fémur. Celui-ci est élargi dans sa partie distale ; il porte du côté antérieur trois articles normaux, bien qu'assez grêles, et du côté 
postérieur, une protubérance conique, non articulée, ébauche d'un deuxième tibia (Q 3) (fig. 115).

$3^{\circ}$ Six nymphes au $2^{\circ}$ stade, gorgées 48 heures auparavant, sont soumises aux vapeurs pendant $2 \mathrm{~h}$. 15. L'extrémité des pattes I est rouge ; une meurt, trois muent le $24^{\circ}$ jour, une le $34^{\circ}$ jour et une le $37^{\circ}$ jour. Deux d'entre elles ont une patte antérieure plus grêle avec l'extrémité du tarse élargie; la première patte droite d'une

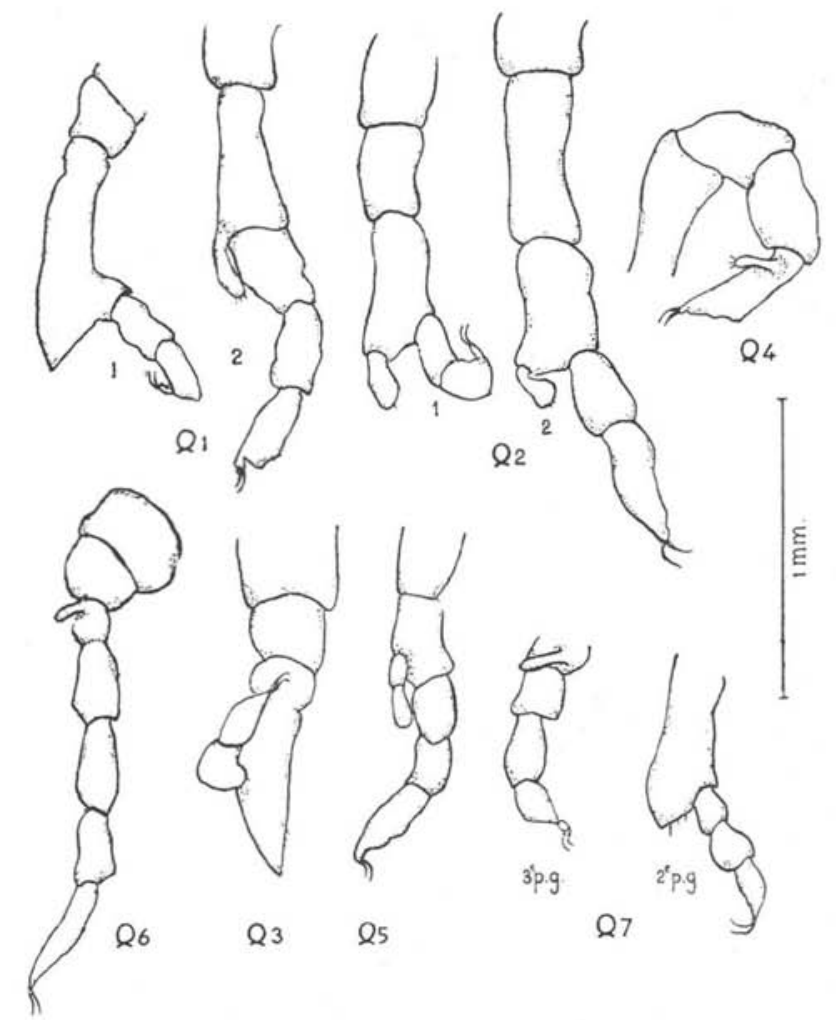

Fig. 115. - Ornithodorus parkeri. Schizomélies obtenues par action de l'éthylquinone sur des nymphes au $2^{\circ}$ stade. Pour $Q 1$ et Q2, persistance de l'anomalie pendant deux stades.

troisième (mue du $24^{\circ}$ jour) est bifurquée; tous les articles sont normaux, sauf le tibia qui porte du côté postérieur une deuxième articulation où s'insère une expansion chitineuse conique à son extrémité et portant trois poils. A la mue suivante, cette formation est devenue membraneuse et plus grêle (Q 2) (fig. 115). 
$4^{\circ}$ Cinq nymphes du même lot sont laissées seulement $1 / 2$ heure. Une meurt, une mue le $18^{\circ}$ jour, une le $19^{\circ}$ jour et deux le $20^{\circ}$ jour. L'une de ces dernières présente une bifurcation du fémur à la première patte droite, postérieurement. Comme précédemment, cet article est beaucoup plus large à son extrémité distale, l'expansion est longue et mince, non articulée et porte trois poils. A la mue suivante, il n'y a plus qu'un bourrelet épaissi à l'extrémité du fémur (Q 1) (fig. 115).

$5^{\circ}$ Sept nymphes du même lot, gorgées cinq jours auparavant, sont laissées 20 minutes; une mue le $15^{\circ}$ jour, deux le $16^{\circ}$ jour, quatre le $18^{\circ}$ jour. Toutes sont normales.

$6^{\circ}$ Six nymphes, gorgées depuis huit jours, sont laissées 15 heures; toutes meurent.

$7^{\circ}$ Six nymphes, gorgées depuis neuf jours, sont mises en contact avec les vapeurs pendant 2 heures 20 minutes. L'extrémité des premières pattes est rosée ; deux meurent, une mue le $20^{\circ}$ jour, une le $21^{\circ}$ jour, une le $25^{\circ}$ jour, une le $31^{\circ}$ jour. Chez cette dernière, la première patte gauche, normale par ailleurs, porte sur la face inférieure du fémur deux articles peu chitinisés, dirigés postérieurement (Q 5) (fig. 115).

$8^{\circ}$ Six nymphes du même lot sont laissées $1 \mathrm{~h}$. 15 . Cinq muent le $20^{\circ}$ jour, une le $21^{\circ}$ jour présente une bifurcation de la troisième patte gauche; les articles sont normaux, seul le tarse présente une protubérance latérale portant trois poils à son extrémité (Q 4) (fig. 115).

\section{Action de la solution alcoolique :}

Nous avons dissous à saturation des cristaux d'éthylquinone dans l'alcool à $95^{\circ}$ et nous avons badigeonné avec cette solution une ou deux pattes à dix nymphes au troisième stade, nourries la veille. Toutes ont mué normalement et aucune ne présentait d'anomalies.

\section{Expériences sur les Ixodidæ}

\section{$1^{\circ}$ Action sur les larves de Hyalomma excavatum}

Nous avons exposé des lots de 50 et 100 larves à l'action des vapeurs d'éthylquinone pendant les temps suivants : 20', 25', 30', $35^{\prime}, 40^{\prime}, 45^{\prime}, 1$ h. 5,1 h. 35,1 h. 45 . Nous n'avons obtenu aucune bifurcation d'appendices. Par contre, nous avons pu relever quelques atrophies des derniers articles des pattes. L'action toxique paraît nulle au-dessous de 35 minutes d'exposition, moyenne entre 35 minutes et 1 heure, léthale au-dessus. Les larves exposées plus 
d'une heure meurent pour la plupart; les quelques exemplaires survivants muent très tardivement, mais ne présentent pas d'anomalies. Sur 100 larves exposées 35 minutes, 30 présentent des malformations portant sur la quatrième paire de pattes ; l'extrémité du tarse est arrondie, raccoureie et ne porte pas toujours de griffes. Cette action toxique est d'ailleurs irrégulière, car, dans des expériences comparables, nous n'avons eu qu'une ou deux malformations ; une seule fois, les tarses de la première paire de pattes étaient absents. Nous pensons qu'il ne s'agit pas ici de véritables anomalies, mais plutôt d'accidents dus au fait que les nymphes ont eu plus de difficultés à sortir de l'exuvie que dans les cas normaux.

Sur 100 larves exposées 45 minutes, 13 seulement ont mué ; l'une d'elles présente une asymétrie marquée de l'écusson et il lui manque la première patte droite (fig. 116).

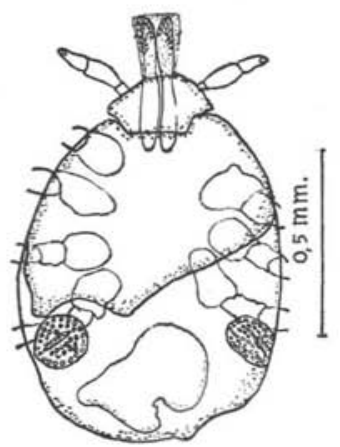

Fic. 116. - Hyalomma excavatum. Nymp he. Ectromélie de la $1^{\text {re }}$ patte droite et asymétrie de l'écusson après action de l'éthylquinone.

\section{$2^{\circ}$ Action sur les nymphes de Dermacentor pictus}

Action des cristaux :

a) Douze nymphes sont mises en contact $1 / 2$ heure avec des cristaux pulvérisés. Toutes meurent avant de muer.

b) Six nymphes sont laissées 12 minutes. Cinq muent normalement et donnent quatre femelles et un mâle; la sixième est une femelle naine ( $3 \mathrm{~mm}$. au lieu de $5 \mathrm{~mm}$.), dont l'écusson est mal délimité et peu chitinisé.

Action des vapeurs :

a) Douze nymphes sont laissées 45 minutes. Trois meurent, les neuf autres donnent des adultes normaux.

b) Six nymphes sont laissées $1 \mathrm{~h} .45$ et muent normalement. 


\section{Conclusions}

L'éthylquinone a une action toxique très nette sur les Ixodoidea. Seules, les expériences sur les Argasidæ ont donné des résultats intéressants. Le contact direct de cristaux ou de poudre provoque généralement la mort des individus, même s'il est peu prolongé ; cependant, nous avons obtenu deux cas de schizomélie, dont une multiple.

L'emploi des vapeurs, qui a l'avantage d'être plus précis, a une action plus ménagée : les animaux, s'ils meurent après avoir passé la nuit dans le tube d'exposition aux vapeurs, résistent bien à des contacts de une à deux heures; sur 42 exemplaires ainsi traités, six seulement meurent et cinq, soit $10,2 \%$, présentent des bifurcations.

Le siège des schizomélies est variable, mais situé le plus souvent au niveau du fémur ou du tibia et à la première paire de pattes. Peut-être pourrait-on voir là un fait relatif à la présence de l'organe de Haller, organe olfactif ; dans les cas où les Tiques ont été exposées un temps suffisamment long, l'extrémité des premières pattes, correspondant au siège de cet organe, prend une coloration rosée ou rouge, indiquant une atteinte plus importante de cette région.

La condensation des vapeurs peut se faire n'importe où, mais les particules qui ont pu pénétrer dans la chambre de l'organe de Haller y restent, ce qui leur permet une action plus prolongée sur le membre correspondant.

Les dédoublements d'appendices obtenus par ce procédé ne sont souvent qu'ébauchés et n'atteignent jamais la perfection de certaines schizomélies obtenues par brûlure. Les résultats obtenus n'en sont pas moins intéressants, car il s'agit de la reproduction d'un processus naturel. Chapman a trouvé des cas de schizomélies et d'anomalies diverses dans des élevages de Tribolium confusum en milieu confiné, et a attribué ce phénomène au rôle toxique de la sécrétion des adultes.

La sécrétion des glandes hypodermiques des Tiques aurait peut-être une influence analogue dans certains cas. 
EXPÉRIENCES SUR O. parketi AVEC LES VAPEURS D'ÉTHYLQUINONE

\begin{tabular}{|c|c|c|c|c|c|c|}
\hline $\begin{array}{c}\text { Nombre } \\
\text { de } \\
\text { tiques }\end{array}$ & $\begin{array}{c}\text { Temps } \\
\text { dexposition }\end{array}$ & Mortalité & $\begin{array}{l}\text { Nombre de } \\
\text { schizomélies }\end{array}$ & $\begin{array}{l}\text { Siège de la } \\
\text { schizomélie }\end{array}$ & $\begin{array}{c}\text { Article } \\
\text { intéressé }\end{array}$ & \\
\hline 7 & 0 h. 20 & 0 & 0 & - & - & - \\
\hline 5 & 0 h. 30 & 1 & 1 & I d. & Fémur & Q 1 \\
\hline 6 & $1 \mathrm{~h}$. & 0 & 0 & - & - & \\
\hline 6 & 1 h. 15 & 0 & 1 & III g. & Tarse & Q 4 \\
\hline 6 & 2 h. 15 & 1 & 1 & I d. & Tibia & Q 2 \\
\hline 6 & 2 h. 20 & 2 & 1 & I g. & Fémur & Q 5 \\
\hline 6 & 2 h. 30 & 2 & 1 & I d. & Fémur & Q 3 \\
\hline 6 & $15 \mathrm{~h}$. & 6 & - & - & - & \\
\hline
\end{tabular}

\section{Action de l'acide sulfurique :}

Avec une pipette effilée, nous avons déposé une petite goutte d'acide sulfurique pur sur la hanche de huit nymphes d'O. parkeri au quatrième stade, gorgées depuis six jours, en prenant pour chacune une patte différente. Aucune n'est morte. Trois ont mué le $15^{\mathrm{e}}$ jour, deux le $20^{\circ}$, trois le $28^{\circ}$ jour ; les cinq premières sont tout à fait normales, les trois autres présentent des anomalies :

a) La troisième patte droite touchée par l'acide est entière, mais petite, la quatrième n'a que trois articles.

b) La deuxième patte gauche est filiforme, la première patte qui avait été légèrement atteinte est représentée seulement par un moignon.

c) La deuxième patte droite est filiforme et se dessèche rapidement. Après montage, nous constatons qu'elle est bifurquée au niveau du fémur (fig. 117) ; la branche secondaire possède trois articles de petite taille, terminés par un pulville et des griffes. La troisième patte qui avait été atteinte également est absente. Cet animal avait été le seul à réagir au moment de l'application de l'acide en émettant un peu de liquide coxal ; l'acide avait dû pénétrer par une articulation et produire une brûlure comparable à celles obtenues par thermocautère. Autrement, la cuticule n'est pas attaquée. Nous pensons donc qu'il s'agit en réalité d'une cautérisation plutôt que d'une action chimique. 


\section{GREFFES}

Les transplantations d'organes ont été tentées assez récemment chez les Insectes. Ce sont surtout les travaux de Bodenstein (1933) qui nous intéressent : l'auteur fait des greffes variées sur des chenilles de Lépidoptères et tire d'importantes conclusions sur la détermination chez la larve de futures formations de l'adulte. Il obtient fréquemment des formations multiples quand le greffon est réimplanté après rotation autour de son axe.

Furukawa (1935-1940) obtient des résultats analogues sur des Forficules. Mauser (1935-37) note l'apparition de formations multiples chez Carausius morosus après transplantation et inversion du greffon. Nous ne parlerons pas des autres expériences qui ne concernent pas les régénérations multiples.

Balazuc (1948), opérant sur des larves de Tenebrio molitor, réussit des greffes orthotopiques et hétérotopiques.

Il emploie des larves assez âgées qu'il opère pendant l'inter-mue en évitant les premiers et les derniers jours. Les animaux sont anesthésiés assez fortement à l'éther et le greffon enchâssé à une certaine profondeur dans le support, les épines et le coagulum suffisant à maintenir le tout solidement en place. Les greffes autoplastiques réussissent bien, tandis que les greffes hétéroplastiques échouent. Il réalise les dix combinaisons suivantes :

\begin{tabular}{c|lll}
\multirow{2}{*}{ Orthotopiques } & Homopleurales & Dorso-dorsale & I \\
& & D-V & II \\
Patte sur moignon de patte & & D-A & III \\
& & D-P & IV \\
& & Proximo-distale & V \\
Hétérotopiques & Hétéropleurales & D-D & VI \\
Patte sur abdomen & Homopleurales & D-V & D-A \\
Antenne sur moignon de patte & - & D-P & VIII \\
& & & IX \\
& & & X
\end{tabular}

Les résultats sont extrêmement intéressants. Il y a un effet local : soit apparition d'un membre d'orientation normale, mais de taille réduite, soit formation d'une schizomélie ternaire, disparaissant au cours des mues suivantes. En outre, il apparaît parfois dans le cas de greffes hétérotopiques (pattes sur abdomen) deux membres surnuméraires séparés, très rudimentaires et disparaissant ensuite. La greffe d'antenne sur moignon de patte donne une formation sim- 
ple, terminée par une antenne qui ne se résorbe pas par la suite, ou une formation triple comprenant une patte et deux antennes.

Effet à distance. - Les opérations ayant porté sur la patte mésothoracique droite, il apparaît parfois sur la patte métathoracique du même côté une schizomélie binaire.

L'auteur compare ces formations triples avec les schizomélies ternaires naturelles. Il estime que le problème est loin encore d'être résolu et suggère que, dans les schizomélies naturelles, une translocation subie par une ébauche d'appendice sous une influence quelconque ait pu faire jouer à celle-ci le rôle du greffon.

Pour l'induction à distance des schizomélies, l'auteur hésite à croire que la perturbation dysharmonique puisse franchir l'intervalle séparant deux somites et se demande s'il s'agit d'un résultat de traumatisme ou d'un phénomène réellement lié à l'inversion de la symétrie ; un fait en faveur de cette dernière hypothèse est la constance de la localisation sur la patte immédiatement postérieure à celle qui a subi le traumatisme.

\section{Expériences personnelles}

\section{Greffes orthotopiques :}

Nous avons utilisé des nymphes d'O. parkeri au dernier stade. Après anesthésie profonde à l'éther, de façon à empêcher tout mouvement de l'animal, nous sectionnons une patte à un niveau variable et la réimplantons dans le moignon après résection d'un article ou deux pour permettre l'introduction assez profonde du greffon. Nous inversons celui-ci de 90 ou $180^{\circ}$. La coagulation de l'hémolymphe suffit généralement à maintenir le greffon en place. L'opération est assez délicate, car l'issue d'hémolymphe est souvent trop importante et les animaux meurent. Il faut pouvoir enchâsser le greffon assez rapidement et, ici, nous n'avons pas de griffes pour le maintenir en place ; en outre, le diamètre du moignon est généralement, malgré la résection d'un ou deux articles, à peine supérieur à celui du greffon.

Nous avons cependant réussi un certain nombre d'opérations. Nous ne les avons pas entreprises sur une grande échelle, car ce qu'il nous importait était de savoir si l'on pouvait obtenir des résultats comparables chez les Insectes et les Acariens, ce que nous avons démontré. Nous n'avons pas obtenu de schizomélie à distance.

\section{Greffes homopleurales :}

Sur 17 nymphes, 4 sont mortes, 10 ont régénéré normalement comme dans le cas d'une section ordinaire, la patte ayant un aspect 
et une direction normaux, mais étant de taille plus ou moins réduite. Nous pensons qu'ici la greffe n'a pas réellement pris, bien que le greffon soit resté en place, sans se dessécher ; il n'a en tout cas pu agir sur le régénérat.

Un individu a régénéré une patte normale, mais dirigée en sens opposé. Il s'agissait de la deuxième patte gauche à laquelle nous avions implanté le tibia inversé à $180^{\circ}$ dans le trochanter. Un autre a donné une schizomélie binaire de la première patte gauche après section du fémur et implantation dans le trochanter à $180^{\circ}$. Le trochanter est élargi dans sa partie distale et porte, dirigée antérieurement, une ébauche d'article et postérieurement quatre articles petits, mais normaux; cette disposition rappelle celle des pattes doubles que nous avons obtenue par brûlure (fig. 118).
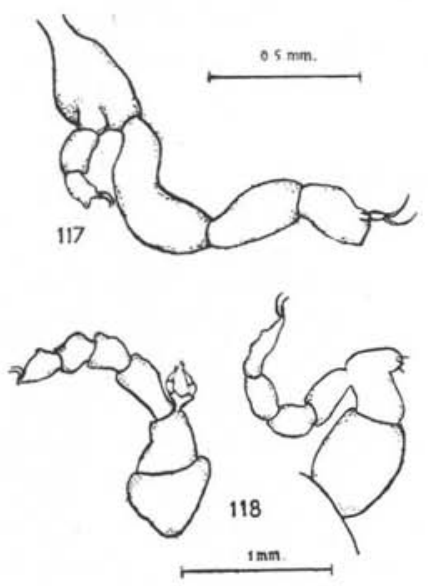

FiG. 117. - Ornithodorus parkeri. Schizomélie obtenue par action de l'acide sulfurique ; FIG. 118. - Ornithodorus parkeri. Résultats obtenus par greffes homopleurales et inversion du greffon à $180^{\circ}$. A gauche, schizomélie ternaire typique.

Enfin, après implantation du tibia inversé à $180^{\circ}$, dans le trochanter de la première patte gauche, nous avons obtenu une schizomélie ternaire typique, dont les trones primaire et secondaire simple donnent l'aspect d'une patte normale; le secondaire double partant de la base du fémur est très court et donne naissance à deux tertiaires de même taille, petits, comprenant trois articles et obéissant à la loi de Bateson (fig. 118).

\section{Greffes hétéropleurales:}

Nous n'avons obtenu que deux résultats positifs, la mortalité étant plus forte ici; l'une a régénéré normalement, l'autre donne une patte normale, mais quelque peu déviée. 


\section{Greffes hétérotopiques :}

Nous avons essayé d'implanter des pattes dans l'abdomen de plusieurs nymphes. Les greffes se sont quelquefois maintenues, mais après la mue nous n'avons jamais observé autre chose qu'une dépression ou une boursouflure de la cuticule, sans trace d'appendice.

\section{RESUME ET CONCLUSIONS}

\section{Tératologie naturelle :}

La tératologie des Tiques n'ayant jamais fait l'objet d'un travail d'ensemble, nous avons cru utile de grouper les différentes monstruosités connues ou inédites. Nous les avons classées en deux grands groupes: anomalies générales portant sur l'ensemble du corps et comportant principalement les gynandromorphes, les monstres doubles et asymétriques; anomalies locales concernant soit un organe tel que stigmate, œil, écusson, feston, soit un appendice qui peut être atrophié, absent (ectromélie) ou divisé (schizomélies diverses).

Nous avons recherché l'origine de ces anomalies : les gynandromorphes mi-partis ou en mosaïque sont probablement le résultat de l'élimination plus ou moins précoce d'un chromosome X. Pour l'intersexué (Hyalomma savignyi) que nous avons trouvé classé primitivement parmi les gynandromorphes, l'origine est certainement hormonale et non germinale.

Les monstres doubles ne proviennent pas, comme les Phasmes de Cappe de Baillon, d'une fusion de l'œuf, mais d'un dédoublement de l'embryon à un stade précoce. Ce dédoublement est toujours postérieur chez les Tiques et chez les Scorpions, tandis qu'il est presque toujours antérieur chez les Insectes. Cette différence est sans doute en rapport avec les possibilités de survie de l'embryon qui ne sont pas les mêmes pour ces deux classes d'Arthropodes.

Les anomalies des appendices ont dans la plupart des cas une cause externe, traumatique, contrairement à l'opinion de divers auteurs.

\section{$2^{\circ}$ Tératologie expérimentale :}

Nous avons essayé divers procédés mécaniques, physiques et chimiques sur les œufs, les larves et les nymphes de plusieurs espèces de Tiques. Seuls, les Argasidæ nous ont donné des résultats positifs. 
Les procédés mécaniques : centrifugation ou morcellement de l'œuf, écrasement ou section d'appendices chez la nymphe, n'ont jamais provoqué d'anomalies.

Les cautérisations partielles d'appendices nous ont par contre donné avec régularité des régénérations anormales du type schizomèle. Les cas de schizomélies binaires ainsi obtenues présentent des branches inégales ou égales obéissant aux lois de symétrie de Bateson ; elles persistent pendant toute la vie de l'individu, contrairement à ce que l'on voit chez les Insectes ; elles ne sont cependant pas héréditaires. Nous avons pu observer quelques cas de schizomélie induite ; dans certaines circonstances, la patte brûlée ne régénère pas, mais la patte voisine est bifurquée.

Nous avons également obtenu des schizomélies avec des produits chimiques : acide sulfurique et éthylquinone, agissant le premier par causticité, le deuxième par toxicité.

La diversité des moyens employés permet de penser que les schizomélies naturelles ont, elles aussi, une cause externe.

Les rayons $\mathrm{X}$ ont une action léthale, mais non tératogénique, sur les œufs et les larves. Les survivants de nos expériences ont un comportement normal et leur descendance ne présente pas de mutants.

L'action des rayons parait s'exercer sur le processus de la mue : les larves gorgées irradiées précocement ne muent pas, les larves irradiées tardivement muent et les nymphes au premier stade peuvent se nourrir, mais ne muent pas.

Des coupes histologiques de larves irradiées ne montrent pas de destructions d'organes. C'est done la production hormonale qui est atteinte.

Enfin, les greffes d'appendices, malgré les difficultés d'expérimentation, ont déterminé l'apparition des schizomélies binaires et ternaires typiques.

Il résulte de ces expériences que l'on ne peut actuellement agir sur la constitution chromosomique, ni sur le développement embryonnaire des Tiques; il existe par contre de nombreux agents producteurs d'anomalies des appendices.

Il est curieux de constater que, dans la nature, on trouve fort peu d'anomalies des appendices, surtout en ce qui concerne les schizomélies, et que la plus forte proportion de monstres relève d'une anomalie embryonnaire. La contradiction avec nos expériences n'est peut-être qu'apparente : en effet, le nombre de Tiques récolté dans la nature est faible par rapport à celui des animaux d'élevage qui se trouvent, de ce fait, soustraits à l'influence de la 
plupart des agents extérieurs; parmi ces derniers, on ne trouve guère que des monstres d'origine embryonnaire. La proportion est inversée chez les Insectes où le nombre d'exemplaires de collection est certainement bien supérieur à celui des animaux d'élevage.

\section{REMERCIEMENTS}

Nous remercions tous ceux qui, dans des domaines divers, nous ont apporté leur aide au cours de ce travail.

Nous exprimons toute notre reconnaissance à M. le Docteur Djourno qui nous a accueillie avec une grande amabilité au Laboratoire de Physique de la Faculté de Médecine; MM. Lacassagne et Latarjet qui nous ont ouvert les portes de l'Institut du Radium ; M. Morenne qui nous a aidée avec beaucoup de patience pour nos irradiations.

Nous remercions également M. le Docteur J. Balazuc, notre Maître en Tératologie, qui ne nous a pas ménagé les précieux renseignements qu'il possédait; M. de Aboïm-Inglez qui nous a prêté tous ses exemplaires tératologiques; M. J. A. T. Santos Dias qui nous a envoyé de Mozambique beaucoup de détails intéressants.

Nous remercions enfin bien sincèrement nos amis, M. le Professeur agrégé A.-G. Chabaud, Mme A. Capaul, Mlles J. et D. Murer pour leur amicale et utile collaboration.

\section{BIBLIOGRAPHIE}

\section{Première partie}

Abom-Inglez (A. L. DE). - Monstruosidades nos Ixcdineos. Vinte e seis monstres na primeira geraçao dum Haemaphysalis cinnabarina var. punctata. (Canestrini et Fanzago 1877). Boletim Pecuario, I, 1942, p. 15-30.

Alfeier (N. I.), - Une nouvelle excroissance anormale chez la femelle de la Tique Hyalomma dromedarii Koch. Zool. Journ., XXVII, n 3, 1948, p. 257-259 (en russe).

Arendsen Hein (S. A.). - Studies en Variation in the Meal-Worm Tenebrio molitor L. II. Variations in tarsi and antenna. Journ. of Genetics, XIV, 1924 , p. 1-38.

Balazuc (J.). - La tératologie des Coléoptères. Mém. Mus. Nat. Hist. Nat., XXV, 1948, p. 1-293.

Bateson (W.). - Materials for the study of Variation, Londres, Mac Millan \& $\mathrm{C}^{\circ}, 1894,598 \mathrm{p}$.

Beaurepatre-Arago (H. DE). - Contribuiçao para a sistematica e biologia dos Ixodidas. Mém. Inst. Osuv. Cruz, IV, 1912, p. 96-118.

Berland (L.). - Sur un Scorpion muni de deux queues. Bull. Soc. Ent., X, 1913, p. 251-252.

Ann. de Parasitologie, T. XXXIII, No $3,1959$. 
BonNET (A.). - Recherches sur l'anatomie comparée et le développement des Ixodidés. Ann. Univ. Lyon, nouvelle série, 1, fase. XX, 1907, p. 1-171.

Boveri (Th.). - Ueber partielle Befruchtung. Sitzungsber. d. Gesell. f. Morphol. u. Physiol. i. München, IV, 1888.

Ueber die Entstehung der Eugsterchen Zwitterbienen. Arch. f. Entwicklungs, XLI, 1915 .

Brauer (A.). - Ueber Doppelbildungen des Skorpions (Euscorpius carpathicus L.). Stizungsber. d. Konigl. Preuss. Akad. D. Wissensch., XII, 1917, p. 208.

Bridges (C. G.). - Elimination of chromosome due to a mutant (minute-n) in Drosophila melanogaster. Proc. Nat. Acad. Sc., XI, 1925, p. 701-706.

Brumpt (E.). - Le gynandromorphisme chez les Ixodinés. Un curieux cas obtenu, dans un élevage d'Amblyomma dissimile. Ann. Parasit., XII, 1934, p. 98-104.

Un mâle monstrueux d'Amblyomma dissimile à deux anus obtenu dans un élevage. Description de divers autres cas tératologiques observés chez les Ixodinés. Ann. Parasit., XII, 1934, p. 105-115.

Précis de Parasitologie, $3^{\circ}$ édition, Paris, Masson et $\mathrm{C}^{\mathrm{ie}}, 1922$.

Campos (F.). - Caso de aberraçion bicaudata en el Centrurus margaritatus Gerv. Rev. Chil. Hist. Nat., XXX1V, 1930, p. 290.

Cappe de Ballon (P.). - Monstres doubles et intersexués chez les Phasmidés. C.R. Acad. Sc. Paris, CLXXX, 1925, p. 1790.

Recherches sur la tératologie des Insectes. Encycl. Entom., VIII, 1927, 291 p.

Diplogénèses et formations multiples chez les Insectes. Bull. Biol. Fr. Belg., LXIII, 1929, p. 456-485.

Tératologie comparée du Plactynemis pennipes Pall. (Odonates Agriodinæ) et du Carausius morosus Br. (Orthopt. Phasmidæ). Bull. Biol. Fr. Belg., LXVII, 1933, p. 1-33.

Crrny (W.). - Anomalies chez quelques Ixodide d'Europe centrale. Acta Soc. Entomol. Cechoslov, LIV, n० 2, 1957, p. 162-171, 20 fig. (en tchèque, résumé russe et allemand).

Снabaud (А.-G.) et Choquet (M.-Th.). - Allométrie des variants sexuels chez les Ixodida. Arch. Zool. Expér. et Gén., LXXXIX, Notes et Revues, n 4 , 1953 , p. $139-146$.

Cooper (W. F.) \& Robinson (L. E.). - On six new species of Ixodidæ, including a second species of the new genus Rhipicentor N. et W. Proc. Cambr. Philos. Soc., XIV, 1908, p. 457-470.

Cunliffe (N.). - The variability of Rhipicephalus pulchellus (Cerstacker, 1873). Parasitology, vol. VI, 1913, p. 204-216.

Rhipicephalus sanguineus : variation in size and structure due to nutrition. Parasitology, VI, 1914, p. 372-378.

Dallas (E. D.). - Ensage de una clasificacion de los Coleopteros anormales, Buenos-Aires, Impr. La Verdad, 1923, 7 p.

DELPY (L.). - Sur la tératologie du sous-genre Hyalomma (Koch 1884). Ann. Parasit. Hum. et Comp., XIV, 1936, p. 48-54.

Despontes (C.). - A propos d'une nymphe monstrueuse de Hyalomma mauritanicum Sénevet. Ann. Parasit., XVI, 1938, p. 11-16.

Doncaster (L.). - On the Relation between Chromosomes, Sex-Limites transmission and Sex-Determination in Abraxas Grossulariata. J. of Genetics, IV, 1914.

Ewest (A.). - Struktur und erste Differenrierung inn Ei des Mehlküfert, Tenebrio molitor. Zeitschr. f. Wissensch. Biol., CXXXV, fasc. 4, 1937, p. 689-752.

Feldman-Muhsam (B.). - On some abnormalities in Hyalomma savignyi. Parasitology, $\mathrm{XL}, \mathrm{n}^{\circ} 1-2,1950$, p. 93-95.

FERwerda (F. F.). - Genetische Studien am Mehlkäfers Tenebrio molitor. S'Gravenhage, Martinus Nijhoff, 1928, 110 p. 
FonsecA (F. DA). - Gymnandromorphismo em Amblyomma cajennense (Fabr. 1787). Notas de Acareologia. Mém. Inst. Butantan, X, 1935-1936, p. $39-41$.

Gadeau de Kerville (H.). - Sur la furcation tératologique des pattes, des antennes et des palpes chez les Insectes. Bull. Soc. Ent. Fr., IV, 1898, p. 93-95.

GÉoffroy SAINT-HiLAIRE (I.). - Histoire générale et particulière des anomalies de l'organisation chez l'Homme et les Animanx, Paris, Baillière, 1837, 3 vol.

Goldschmidt (R.). - Le déterminisme du sexe et l'intersexualité, Félix Alcan, $1932,192 \mathrm{p}$.

et KatsuKi (K.). - Cytologie des Erblichen Gynandromorphismus von Bombyx mori L. Biol. Zentralblatt, XLVIII, 1928.

GuYÉnor (E.). - L'hérédité, 3e édition, Doin et C'1e, 1942, 718 p.

Hoge (M. A.). - The influence of temperature on the development of a mendelian character. J. of Exp. Zool., XVIII, 1915, p. 241-295.

JoAN (T.). - Caso de gynandromorfismo en una garrapata Amblyomma neumanni Rib. Prim. Reunion Nac. Soc. Argent. Ciencias Nat. Tucuman (1916), Buenos-Aires, 1919, p. 421-425.

Krause (G.). - Analyse erster Differenzierungsprozesse im Keim der Gewãchshausdeuschrecke durch künstlich erzeugte Zwillings. - Doppel und Mejrfschbildungen. Zeitschr. f. Wiss. Biol., CXXXII, fasc. 1, 1934, p. $115-205$.

LENGERKEN (H. V.). - Ueber die Entstehung bilateral-symmetrischer Insektengynander aus verschmolzenen Eier. Biol. Zentralblatt, XLVIII, 1928, p. 475-509.

Lounsbury (C. P.). - The fowl tick. Studies on its life-cycle and habits. Agric. Journ. Cape Town, IX, 1903, p. 1-15.

Morgan (T. H.). - An alternative interpretation of the Origin of gynandromorphous Insects. Science, XXI, 1905.

Mosaics and gynandromorphs in Drosophila. Proc. Soc. Exper. Biol. Med., XI, 1914 .

\& Dridges (C. B.). - The origin of gynandromorphs, Carnegie Inst. Wash. Publ., CCLXXVIII, 1919, p. 1-122.

Neumann (G.). - Anomalies d'Ixodidés. Arch. Parasit., II, 1899, p. 463-465.

Nordenskiol. (E.). - Spermatogenesis in Ixodes ricinus L. Parasitology, XII, 1920 , p. $159-166$.

Nuttall (G. H. F.). - Tich abnormalities. Parasitology, VII, 1914-1915, p. $250-257$.

Olenev (N. O.). - Teratologische Erscheinungen bei den Zecken (Ixodoidea). Zool. Anzeiger, XCXIII, 1931, p. 281-284.

Oppermann (E.). - Die Entstehung der Riesenspermien von Argas columbarum (Shaw) (reflexus f.). Zeitschr. Mikr. Anat. Forschg., XXXVII, 1935, p. 538-560.

Oudemans (A. C.). - Amblyomma scaevola nov. sp. Entomol. Berichten, XXII, 1905 , p. 216.

Patterson (J. T.). - The effects of X-rays in producing mutations in the somatic cells of Drosophila melanogaster. Science, LXVIII, 1928, p. 41-45.

- X-rays and somatic mutations. J. of Hered., XX, 1929, p. 261-267.

PAvesi (P.). - Toradelfia di uni Scorpione. R.C. R. Inst. Lombardo, série 2, XIV, 1881, p. 329.

PAvlovsky (E. N.). - Monstrosities and abnormalities in the ticks Ixodoidea. Mag. Parasit. Inst. Zool. Acad. Sci. U.R.S.S., VII, 1940, p, 7-44.

et Bernadskaia (M.). - Nouveaux gynandromorphes chez les Tiques Ixodoidea. Mag. Parasitol., X, 1948, p. 25-40, 19 fig. (en russe).

Pereira (C.) \& Castro (M. P. DE). - Sobre un ginandromorfo de Rhipicephalus sanguineus Latr. 1804. Arc. Inst. Biol. São-Paulo, XVI, 1945, p. 187-192.

Prrvomaisky (G. S.). - Une nouvelle Tique synandromorphe du genre Hyalomma Koch (Acarina, Ixodidæ). Entomol. Obosrenie, XXXI, n ${ }^{\circ} 1-2$, 1950 , p. 113-120, 10 fig. (en russe). 
Variabilité des Tiques de pâturage (Acarina, Ixodidæ) et sa signification pour la systématique. Trudi Entomol. Akad. Sc. U.R.S.S., XI.IV, 1954, p. 63-201, 118 fig. (en russe).

Przibram (H.). - Die Bruch-Dreifachbildung im tierreiche. Arch. f. Entw. Mech. d. Org., XLVIII, 1921, p. 205-444.

RoBrnson (L. E.). - Malformations in ticks. Parasitology, XII, 1920, p. 175-179.

Robinson (G. G.). - Cases of abnormal development in the Argasid tick, Ornithodorus moubata. Parasitology, XXXV, 1943, p. 23-26.

More cases of abnormal development in the Argasid tick, Orrithodorus moubata Murray. Parasitology, XXXVI, 1944, p. 95-97.

Santos Dias (J. A. T.). - Anomalias nos Ixodideos. Moçambique, LV, 1947, p. $5-30$.

Sobre alguns cases teratologicos observados na Amblyomma hebraeum Koch, 1844. Anais dos Servicos de Veterinaria et Industria Animal, I, 1947 , p. $77-93$.

Quelques cas curieux d'anomalies, observés chez les Ixodidés des genres Rhipicephalus et Aponomma. Ann. Parasit., XXIII, 1948, p. 27-30.

Um caso de deformidade num Amblyomma tholloni Neum. 1899, Moçambique, LVI, 1948 , p. 41-44.

Tres casos de malformaçâo observados na especies Amblyomma variegatum (Fabr. 1794). Moçambique, LVI, 1948, p. 47-55.

Un caso de ginandromorfismo observado no Ixodideo Rhipicephalus neavii Warburton 1912. Ann. Inst. Med. Trop. Lisboa, VIII, n ${ }^{\circ} 4$, p. 523-530.

Sobre uma pequena coleç̧ao de carraças provenientes do Mossurize (Manica e Sofala-Moçambique). Rev. Fac. Sc. Lisb., II, n 2, 1952, p. 293304.

- Nova contribuçao para o estudo da Teratologia dos Ixodideos. Ann. Inst. Med. Trop. Lisb., X, 1953, p. 2249-2275, 40 fig.

Schulze (P.). - Ueber Zeckengynander. Zeit. f. Morphol. u. Oekol. der. Tiere, XXVI, 1933 , p. 427-436.

Ein bemerkenswerter Gynander der Zecke Hyalomma detritum damascenium P. Sch. U. Sch., 1929. Sitzungsber. $n$. Abh. der Natur. Ges. Rostock, VI, 1936 , p. $83-88$.

Ein auscheinend durch Eiverschmelzung entstandener Gynander der Zecke Uroboophilus cyclops Minning 1934. Zool. Anz., CXIX, 1937, p. $160-166$.

Ueber Missbildungen der Schildzecken im Allgemeinen sowie über Missbildungen von Hyalomma steineri enigkianum n. sp. im Besonderes. Zeitsch. $f$. Parasitenk., XIV, 1949-1950, p. 545-573, 12 fig.

Seba (A.). - Scorpio brasiliensis cauda bifida. Rerum Naturalium Thesaurus, I, 1734 , p. 12.

Seider (F.). - Die determinierung der Keimanlage bei Insekten, I. Biol. Zentralblatt, XLVI, fasc. 6,1926 , p. $321-343$; id., II ; id., XLVIII, fasc. 4 , 1928 , p. $230-251$; id., III, id., fasc. 10 , p. 577-607.

- Untersuchungen über das Bildungsprinzip der Keimanlage fm Ei Der Libelle Platycnemis pennipes. Zeitschr. $f$. Wissensch. Biol., CXIX, 1929, p. $322-440$.

Selbie (C. M.). - A new variety of Polydesmus coriaceus Porat, and note of a centripede monstrosity. Ann. and Mag. Nat. Hist., XII, 1913, p. 439.

Sénever (G.). Monstruosités chez deux Tiques. Bull. Soc. Hist. Nat. Af. du N., XIII, 1922, p. 95 .

Sergent (Ét.). - Anomalies chez les Scorpions. Arch. Inst. Pasteur Alger, XXIV, 1946 , p. 80 .

et Poxcer (Mme A.). - Observations morphologiques sur la descendance d'une Tique monstrueuse. Arch. Inst. Pasteur Alger, XIX, 1941, p. 432434.

Sharif (M.). - A note on monstruosities observed in Ixodid ticks. Rec. Ind Mus., XXXII, 1930, p. 107-112.

Silva Leitao (J. L. DA). - Contribuiçao para o estudo dos ixodideos portugueses, 1943 (in Santos Dias, 1947). 
Smrrh (C. N.). Gynandromorphism in Ixodes dentatus Marx. Proc. Ent. Soc. Nash., XLIV, 1942, p. 52.

Sovto (G. G.). - Sobre uma interessante anomalia observada em Boophilus microplus (Canest., 1883). Bol. Soc. Brasil. Med. Veter., IX, n 2, 1939, p. $109-110$.

Stankof (O.). - Due case de Malformazione in Ixodida. Riv. di Parassitol., XVII, $\mathrm{n}^{\circ} 3,1956$, p. 189-191, 1 fig.

Tonsier (G.). - Das Entstehen von Käfermissbildungen, besonders Hyperantennie und hypermelie. Arch. f. Entw. Mech. d. Org., IX, 1900, p. 501562.

Tselitcheva (L. M.). - Nouveavx gynandromorphes chez les Tiques du genre Hyalomma. Nouvelles Acad. Sc. Rép. Sov. Kazakstan, sćr. Parasitologie, VI, 1948. (In Pervomaisky, 1954).

Tur (.J.). - Observations sur l'oogénèse chez le Hanreton (Melolontha vulgaris). Bull. Biol. Fr. Belg., LIV, 1920, p. 37-52.

TUzeT (O.) \& Mrlot (J.). - Recherches sur la spermiogénèse des Ixodes. Bull. Biol. Fr. Belg., LXXI, 1937, p. 190-205.

VAchos (M.). - Remarques sur l'arthrogénèse des appendices. A propos d'un cas de symmélie partielle chez un pseudoscorpion, Chelifer cancroides L. (Arachnide). Bull. Biol. Fr. Belg., LXXXI, fasc. 1-2, 1947, p. 177-194.

\& Serfaty (A.). - Remarques sur les Scorpions à deux queues. A propos d'un spécimen anormal appartenant à l'espèce Buthotus alticola (Pocock). Bull. Soc. Zool. Fr., LXXV, n* 2-3, 1950, p. 91-96.

VAxDel. (A.). - Etude d'un gynandromorphe (dinergatandromorphe) de Pheidole pallidula Nyl. (Hyménoptères, Formicidés). Bull. Biol. Fr. Belg., LXV, 1931 , p. $114-129$.

WAGNen (J.). - Die Embryonalentwicklung von Ixodes calcaratus. Trudui St. Peterb. Obshch., XXIV, 1894.

Warbuntox. (C.). - Notes on the genus Rhipicephalus, with the description of new species, and the consideration of some species hitherto described. Parasitology, V, 1913, p. 1-20.

\& Nutrall (G. H. F.), - On new species of Ixodide, with a note on abnormalities observed in ticks. Parasitol., LI, 1909, p. 57-76.

WheELER (W. M.). - Some new gynandromorphs Ants, with a review of the previously recorded cases. Bull. Amer. Muz. Nat. Hist., XIX, 1903.

WhITINg (P. W.) \& Wesstrup (E. I.). - Fertile gynandromorphs in Habrobracon. J. Hered., XXIII, 1932.

Woonworth (C. E.). - A wireworm double monster (Limonius canus Lee., Elaterida). Psyché, Boston, XXXIX, 1932, p. 37-40.

Zapletal (M.). - Eine interessante Anomalie der Zecke Ixodes ricinus L. Zoologické Listy, XX, n ${ }^{\circ} 1,1957$, p. 29-30, 1 fig. (en tchèque, résumé allemand).

\section{BIBLIOGRAPHIE}

\section{Deuxième partie}

Abezoos (M.). - Sur la régénération des pattes chez le Coléoptère Timarcha violaceo-nigra De Geer. C.R. Soc. Biol., CXIII, 1933, p. 17-19.

Anomalies des pattes de Timarcha violaceo-nigra De Geer. Bull. Soc. Linnéenne Normandie, VI, $\mathrm{n}^{\circ} 4,1933$, p. 33-34.

Balazuc (J.). - La tératologie des Coléoptères. Mém. Mus. Nat. Hist. Nat., XXV, 1948 , p. 1-268.

Expériences de transplantation sur Tenebrio molitor L. Mém. Mus. Nat. Hist. Nat., XXV, 1948, p. 269-293.

BodensteIn (D.). - Ëperimentell erzeugte Doppelbildungen von Lepidoptere beinen. Zool. Anz., CII, 1933, p. 34-38. 
Beintransplantationen and Lepidopteren-Raripen, I. Transplantationen zur Analyse der Raupen-und Puppenhaütung. Keitschr. f. Wiss., Biol., CXXVIII, 1933, p. 564-583.

Beintransplantationen and Lepidopteren-Raupen. II. Zur Analyse der Regeneration der Brutsbeine von Vanessa urtica-Raupen. Id., CXXX, 1933, p. 747-769.

Beintransplantationen and Lepidopteren-Raupen. III. Zur Analyse der Entwiklung spotenzen der Schmetterlingebeine. Id., CXXXIII, 1935, p. $156-192$.

- Beintransplantationen and Lepidopteren-Raupen. IV. Zur Analyse experimentell erzeugter Bein-Mehrfachbildungen. Id., CXXXVI, 1937, p. 745785 .

Bordage (E.). - Recherches anatomiques et biologiques sur l'autonomie et la régénération chez divers Arthropodes. Bull. Scient. Fr. et Belg., XXXIX, 1905 , p. $307-454$.

Boundox (J.). - Recherches expérimentales sur la régénération chez un Coléoptère. Bull. Biol. Fr. Belg., LXXI, 1937, p. 466-499.

Brindiey (H. H.). - On the regeneration of the legs in the Blattida. Proc. Zool. Soc., 1897, p. 903-916.

On certain characters of reproduced appendages in Arthropoda, particularly on the Blattidx. Proc. Zool. Soc., 1898, p. 924-958.

CaMpana (Y.). - La régénération chez Ornithodorus parkeri et son influence sur la mue. Ann. Parasit., XX, 1944-1945, p. 321-329.

- Un cas tératologique d'origine expérimentale chez Ornithodorus parkert. C.R. Soc. Biol., CXLI, 1947, p. 133.

Schizomélies spontanées et expérimentales chez les Ixodoidea. Ann. Parasit., XXII, 1947, p. 53-62.

Cappe de BAIllox (P.). - La bipartition monstrueuse du pronotum chez les Coléoptères. Ann. Soc. Entom. Fran., CI, 1932, p. 245-266.

ChapmaN (T. A.). - Some experiments on the regeneration of the legs of Liparis dispar L. Second Intern. Congr. Entomol., Oxford, 1913, Hazell, Wateson \& Viney, p. 295-305.

Cux́sơ (L.). - Régénération de pattes à la place d'antennes sectionnées chez un Phasme. C.R. Acad. Sc. Paris, CLXXII, 1921, p. 949-952.

- Sur les différents modes de régénérations des antennes chez le Phasmo Carausius morosus. Id., p. 1009-1011.

Furukawa (H.). - Can the skin of imago be made to molt ? Proc. Imp. Acad. Tokyo, XI, 1935, p. 158-160.

- Transplantation experiments on appendages of Anisolabis maritima (Dermaptera). Jap. J. of Zool., VIII, 1940, p. 479-535.

Gadeau de Kerville (H.). - Expériences tératogéniques sur différentes espèces d'Insectes. Le Naturaliste, XII, 1890, p. 114.

Hindie (E.) \& Cuntrffe (N.). - Regeneration in Argas persicus. Parasitology, VI, 1914 , p. $353-371$.

Hoge (M. A.). - The influence of temperature on the development of a mendelian character. J. Exp. Zool., XVIII, n 2, 1915, p. 241-298.

GEIGY (R.). - Une anomalie non héréditaire provoquée par les rayons ultraviolets chez la Drosophile. C.R. Soc. Phys. et Hist. Nat. Genève, XLIII, 1926 , p. 143-145.

Production d'anomalies par exposition de Drosophiles aux rayons ultraviolets. Actes Soc. Helv. Sc. Nat., $108^{\circ}$ session, 1927, $2^{e}$ partie, p. 191-192.

Action de l'ultra-violet sur le pôle germinal dans l'œuf de Drosophila melanogaster (castration et mutabilité). Rev. Suisse Zool., XXXVIII, 1931 , p. $187-288$.

- Erzeugung rein imaginaler Defekte durch ultraviolette Eibestrahlungbei Drosophila melanogaster. Zeitschr. f. Wiss. Biol., CXXV, 1931-1932, p. 406-447.

\& Luscher (M.). - Imaginale Bein-Doppelbildungen nach UltraviolettBestrahlung von Schmetterlingseiern (Tineola biselliela). Rev. Suisse Zool., XLIX, 1942, p. 206-210. 
JANDA (V.). - Fühlerähnliche Heteromorphosen an Stelle von Augen bei Stylopyga orientalis und Tenebrio molitor. Arch. f. Entuicklungsmechanik der Org., XXXVI, 1913, p. 1-3.

KOPEC (S.). - Untersuchungen über die Regeneration von Larvalorganen und Imaginalschreiben bei Schmetterlingen. Arch. f. Entwick. Mech. d. Org., XXXVII, 1913, p. 440-469.

línzeNecky (J.). - Versuche über die regeneration des Abdominalendes von Tenebrio molitor während seiner post-embryonaler Entwicklung. Arch. f. Entwickl. Medh. d. Org., XXXVI, 1913, p. 294-341.

Matser (F.). - Synchrone Metamorphose deplantierter Vorderbeine mit dem Wirstiere Dixippus morosus. Br. Redt. Histologische Untersuchung der Sabheuschrecker mit replantierten Köpfen. (Mitt. aus d. Biol. Versuchanstalt d. Ak. d. Wiss. Zool, Abtlg., n ${ }^{\circ}$ 230-231, p. 168-169). Anz. Akad. Wiss. Wien., LXXII, 1935, $\mathrm{n}^{\circ} 17$.

Weitere Versuche zur synchronen Metamorphose an der Beinen von Dixippus morosus $\mathrm{Br}$. Redt., id., LXXIV, $\mathrm{n}^{\circ} 17$, 1937, Mitt. $\mathrm{n}^{\circ} 266$, p. $154-155$.

Mrgusan (F.). - Die Regeneration der Coleopteren. Arch. f. Entwick. Mech. d. Org., XXV, 1907 , p. 148-234.

Nutrall (C. H. F.). - Regeneration of the mouthparts and legs in Ticks. Parasitology, XII, 1920, p. 7-26.

Patay (R.). - Sur la régénération des pattes chez le Coléoptère Chrysomélide Leptinotarsa decemlineata Say. C.R. Soc. Biol., CXXVI, 1937, p. 283-285.

Pauliax (R.). - Contribution à l'étude quantitative de la régénération chez les Arthropodes. Proc. Zool. Soc. London, CVIII, 1938, p. 297-383.

Pervomaisky (G. S.). - Variabilité des Tiques de pâturage (Acarina, Ixodida) et sa signification pour la systématique. Trudi Entomol. Akad. Sc. U.R.S.S., XLIV, 1954, p. 63-201, 118 fig.

Poisson (R.) \& Patay (R.). - Sur quelques modalités de la régénération des pattes et des ailes chez la larve du Doryphore : Leptinotarsa decemlineata Say. C.R. Soc. Biol., CXXIX, 1938, p. 126-128.

Przibram (H.). - Fühlerregeneration halberwachsener Sphodromantis-Larven. Arch. f. Entw. Mech. d. Org., XLIII, 1918, p. 63-87.

Regeneration des Fühlers samt Johnston'schem Organe beim Mashornkäfer (Oryctes nasicornis L.). Anz. Akad. Wissens. in Wien., LXVIII, n²6, 1931 .

Rehacek (J.). - The malformations of the Tick Dermacentor marginatus Sulz. Zool. Listy, $\mathrm{XX}, \mathrm{n}^{\circ} 1,1957$, p. 39-40, 1 pl.

Semel (F.). - Die determinierung der Keimanlage bein Insekten, 1. Biol. Zentralblatt, XLVI, 1926, p. 321-343.

Die determinierung der Keimanlage bein Insekten, 2, id., XLVIII, 1928, p. 230-251.

- Die determinierung der Keimanlage bein Insekten, 3, id., p. 577-607.
- Untersuchungen über das Bildungsprinzip der Keimanlage im Ei der Libelle Platycnemis pennipes. Zeitschr. f. Wiss. Biol., CXIX, 1929, p. $322-440$.

Sergent (Edm.) et Poxcet (Mme A.). - Etude expérimentale de la régénération des appendices chez les Tiques (Ordre des Acariens, sous-famille des Ixodinæ). Arch. Inst. Pasteur d'Algérie, XXI, 1943, p. 215-232.

TOnxIER (G.). - Neues über das natürliche Entstehen und experimentelle Erzeugen überzahliger und Zwillingsbildungen. Zool. Anz., XXIV, 1901, p. 488-504.

Bein-und Fühlerregeneration bein Käfern und ihre Begleiterscheinungen., id., XXIV, 1901, p. 649-664.

Werber (I.). - Regeneration des exstirpierten Fühlers und Auges beim Mehlkäfer (Tenebrio molitor). Arch. f. Entw. Mech. d. Org., XIX, 1905, p. 259260 . 